\title{
Boron and phosphorus containing heterosiliconoids: stable p- and n-doped unsaturated silicon clusters
}

Yannic Heider, Philipp Willmes, Volker Huch, Michael Zimmer, David Scheschkewitz*

Krupp-Chair for General and Inorganic Chemistry, Saarland University, 66123 Saarbrücken (Germany),

Supporting Information 


\section{Supporting Information}

\section{Table of Contents}

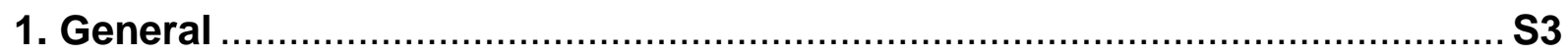

2. Experimental procedures, data and plots of spectra of:

2.1. 2,3,3,4-Tetrakis(2,4,6-triisopropylphenyl)tricyclo[2.1.0.02,5]penta-

silan-1,5-diyl)lithium (4) S3

2.2. 2,3,3,4-Tetrakis(2,4,6-triisopropylphenyl)-1,5-

bis(trimethylsilyl)tricyclo[2.1.0.0 2,5]pentasilane (7) .............................................. 10

2.3. N,N-Diisopropyl-4,5,5,6-tetrakis(2,4,6-triisopropylphenyl)-1,3,4,5,6-pentasila-2-boratetracyclo[2.2.0.0 $\left.0^{1,3} \cdot 0^{3,6}\right]$ hexan-2-amine (8)

2.4. N,N-Diisopropyl-4,5,5,6-tetrakis(2,4,6-triisopropylphenyl)-2-phospha-

1,3,4,5,6-pentasilatetracyclo[2.2.0.0 $0^{1,3} \cdot 0^{3,6}$ ]hexan-2-amine (9) ..........................S17

3. Details on X-ray Diffraction Studies.......................................................... S25

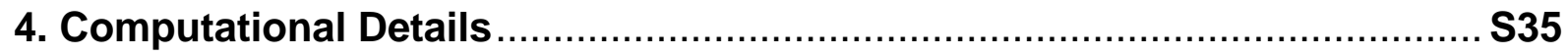

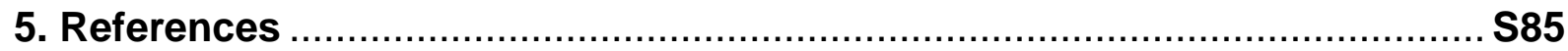




\section{General}

All manipulations were carried out under a protective atmosphere of argon, using Schlenk techniques or a glove box. Benzene was refluxed over sodium/benzophenone for purification. All other solvents used were purified by a PureSolvMD5 solvent purification system by Innovative Technology. Deuterated benzene $\left(\mathrm{C}_{6} \mathrm{D}_{6}\right)$ and toluene- $\mathrm{d}_{8}$ were refluxed over potassium, then distilled and stored under argon. Tetrahydrofuran-d8 was refluxed over $\mathrm{Na} / \mathrm{K}$ alloy, then distilled and stored under argon. UV/Vis spectra were acquired using a Shimadzu UV-2600 spectrometer and quartz cells with a path length of $0.1 \mathrm{~cm}$. NMR spectra were recorded on a Bruker Avance III $300 \mathrm{MHz}$ and/or a Bruker Avance III HD $400 \mathrm{MHz}$ spectrometer. Solid-state NMR spectra were recorded on a Bruker AV400WB spectrometer. Elemental analyses were carried out with an elementar vario Micro Cube. Siliconoid 2 was prepared according to our published procedure. $\mathrm{Me}_{3} \mathrm{SiCl}$ and ${ }^{i} \mathrm{Pr}_{2} \mathrm{~N}-\mathrm{BCl}_{2}$ were purchased from Sigma Aldrich and distilled prior to use. $\mathrm{Pr}_{2} \mathrm{~N}-\mathrm{PCl}_{2}$ was prepared according to a literature known procedure. ${ }^{[1]}$

\section{Experimental procedures, data and plots of spectra of:}

\subsection{2,3,3,4-Tetrakis(2,4,6-triisopropylphenyl)tricyclo[2.1.0.0 2,5]pentasilan-1,5- diyl)lithium (4)}

To a suspension of $3.28 \mathrm{~g}(2,36 \mathrm{mmol})$ of perarylated hexasilabenzpolarene 2 in 30 $\mathrm{mL}$ of $\mathrm{Et}_{2} \mathrm{O}, 20 \mathrm{~mL}$ of a $\mathrm{Li} / \mathrm{C}_{10} \mathrm{H}_{8}$ solution in thf $(0.53 \mathrm{M})$ are added at $-78^{\circ} \mathrm{C}$. The resulting reaction mixture is allowed to slowly warm to room temperature overnight with vigorous stirring. All volatiles are removed in vacuum. The solid residue is dissolved in toluene, filtered and the solvent distilled of in vacuum. The residue is then digested with hexane and subsequently dried again under reduced pressure. Most naphthalene is removed by sublimation at $50^{\circ} \mathrm{C}$ in vacuum. The crude product is washed with a hexane-benzene mixture (3:1). $1.92 \mathrm{~g}(69 \%)$ of the Sis dianion 4 
precipitates from hexane as ochre to olive coloured microcrystalline powder with variable residual amounts of naphthalene and 1,3,5-triisopropylbenzene. Single crystals suitable for an X-ray diffraction study were grown from a hexane/toluene solution after 3 days at r.t. Higher quality single crystals for $\mathrm{X}$-ray diffraction studies were obtained after several weeks from a highly diluted hexane solution.

(m.p. $>220^{\circ} \mathrm{C}$, dec.).

The dianion $\mathbf{4}$ is also accessible by reduction of the monoanionic siliconoid privo-3 with 2.2 equivalents of a $\mathrm{Li} / \mathrm{C}_{10} \mathrm{H}_{8}$ solution in thf. The experimental procedure used is similar to the one described above. Quantities: privo-3: $189.5 \mathrm{mg}(0.14 \mathrm{mmol})$; $\mathrm{Li} / \mathrm{C}_{10} \mathrm{H}_{8}: 0.58 \mathrm{~mL}(0.52 \mathrm{M}, 0.30 \mathrm{mmol})$.

${ }^{1} \mathrm{H}$ NMR $\left(300.13 \mathrm{MHz}, \mathrm{C}_{6} \mathrm{D}_{6}, 300 \mathrm{~K}\right): \delta=7.64-7.61\left(\mathrm{~m}, 2 \mathrm{H}, \mathrm{C}_{10} \mathrm{H}_{8}\right), 7.31$ (d, $2 \mathrm{H}$, TipH), $7.27-7.24\left(\mathrm{~m}, 2 \mathrm{H}, \mathrm{C}_{10} \mathrm{H}_{8}\right), 6.99(\mathrm{~s}, 0.5 \mathrm{H}$, residual $\mathrm{TipH}=1,3,5-$

Triisopropylbenzene), 6.93 (d, 2H, Tip- $H$ ), 6.86 (d, 2H, Tip- $H$ ), 6.83 (d, 2H, Tip- $H$ ), 5.30 (sept, 2H, Tip-'Pr-CH), 4.95 (sept, 2H, Tip-iPr-CH), 4.62 (sept, 2H, Tip-'Pr-CH), 3.69 (br, 6H, thf), 3.24 (sept, 2H, Tip-Pr-CH), 2.86 to 2.69 (m, 4.5H, Tip-Pr-CH and residual TipH), 2.10 (d, 6H, Tip-'Pr-CH3), 1.65 (d, 6H, Tip-Pr-CH3), 1.54 (d, 6H, TipPr- $\mathrm{CH}_{3}$ ), 1.45 (br, 6H, thf), 1.39 (d, 6H, Tip-iPr- $\mathrm{CH}_{3}$ ), 1.25 (d, 21H, Tip-Pr- $\mathrm{CH}_{3}$ and residual TipH), 1.18 (d, 6H, Tip-Pr- $\mathrm{CH}_{3}$ ), 0.74 (br d, 6H, Tip-'Pr-CH $\mathrm{CH}_{3}, 0.67-0.61$ (m, 12H, Tip-IPr-CH $\mathrm{CH}_{3}$ ), 0.37 (d, 6H, Tip-'Pr- $\mathrm{CH}_{3}$ ).

${ }^{13} \mathrm{C}$ NMR $\left(100.6 \mathrm{MHz}, \mathrm{C}_{6} \mathrm{D}_{6}, 300 \mathrm{~K}\right): \delta=157.9,154.8,152.6,151.9,149.1,148.7$, 147.8, 142.3, 134.1 (Tip- $C$ and Tip- $C H), 133.7,128.2,126.1\left(\mathrm{C}_{10} \mathrm{H}_{8}\right), 122.4$ 122.3, 122.2, 121.2, 120.6 (Tip- $C$ and Tip- $C H$ ), 69.8 (thf), 36.5, 36.4, 35.1, 34.8, 34.8, 34.7, 34.1 (Tip-'Pr-CH and Tip-'Pr- $\mathrm{CH}_{3}$ ) 32.0 (hexane), 29.8, 29.6 (Tip-'Pr-CH and Tip-Pr$\mathrm{CH}_{3}$ ), 27.9 (thf), 25.6, 25.2, 24.9, 24.6, 24.5, 24.4, 24.2, 24.0, 23.8 (Tip-Pr- $\mathrm{CH}$ and Tip-Pr- $\mathrm{CH}_{3}$ ), 23.1 (hexane), 22.4 (Tip-'Pr-CH and Tip-Pr- $\mathrm{CH}_{3}$ ), 14.4 (hexane).

${ }^{29} \mathrm{Si} \mathrm{NMR}$ (79.5 MHz, $\left.\mathrm{C}_{6} \mathrm{D}_{6}, 300 \mathrm{~K}\right): \delta=55.7$ (SiTip 2$),-90.1$ (SiTip), -298.3 (Si). ${ }^{29} \mathrm{Si} \mathrm{CP} / \mathrm{MAS}$ NMR $(79.5 \mathrm{MHz}, 13 \mathrm{kHz}): \delta=42.5$ (SITip 2$),-100.5$ (SITip), -290.6 (SI). ${ }^{7} \mathrm{Li} \mathrm{NMR}\left(116.6 \mathrm{MHz}, \mathrm{C}_{6} \mathrm{D}_{6}, 300 \mathrm{~K}\right): \delta=3.74$ (br).

${ }^{7} \mathrm{Li}\left\{{ }^{1} \mathrm{H}\right\}$ CP/MAS NMR $(155.6 \mathrm{MHz}, 13 \mathrm{kHz}): \delta=5.80,2.28,-0.57$.

UV/Vis (hexane): broad shoulders from $\lambda_{\max }=340-390 \mathrm{~nm}$ and $\lambda_{\max }=260-285$ $\mathrm{nm}$.

Elemental analysis: calculated for $\mathrm{C}_{66} \mathrm{H}_{104} \mathrm{Li}_{2} \mathrm{O}_{1.5} \mathrm{Si}_{5}: \mathrm{C}, 73.68 \% ; \mathrm{H}, 9.74 \% ; \mathrm{N}, 0 \%$. Found: C, 73.33; H, 9.32; N, 0 . 
ఈु

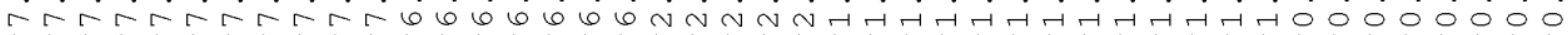
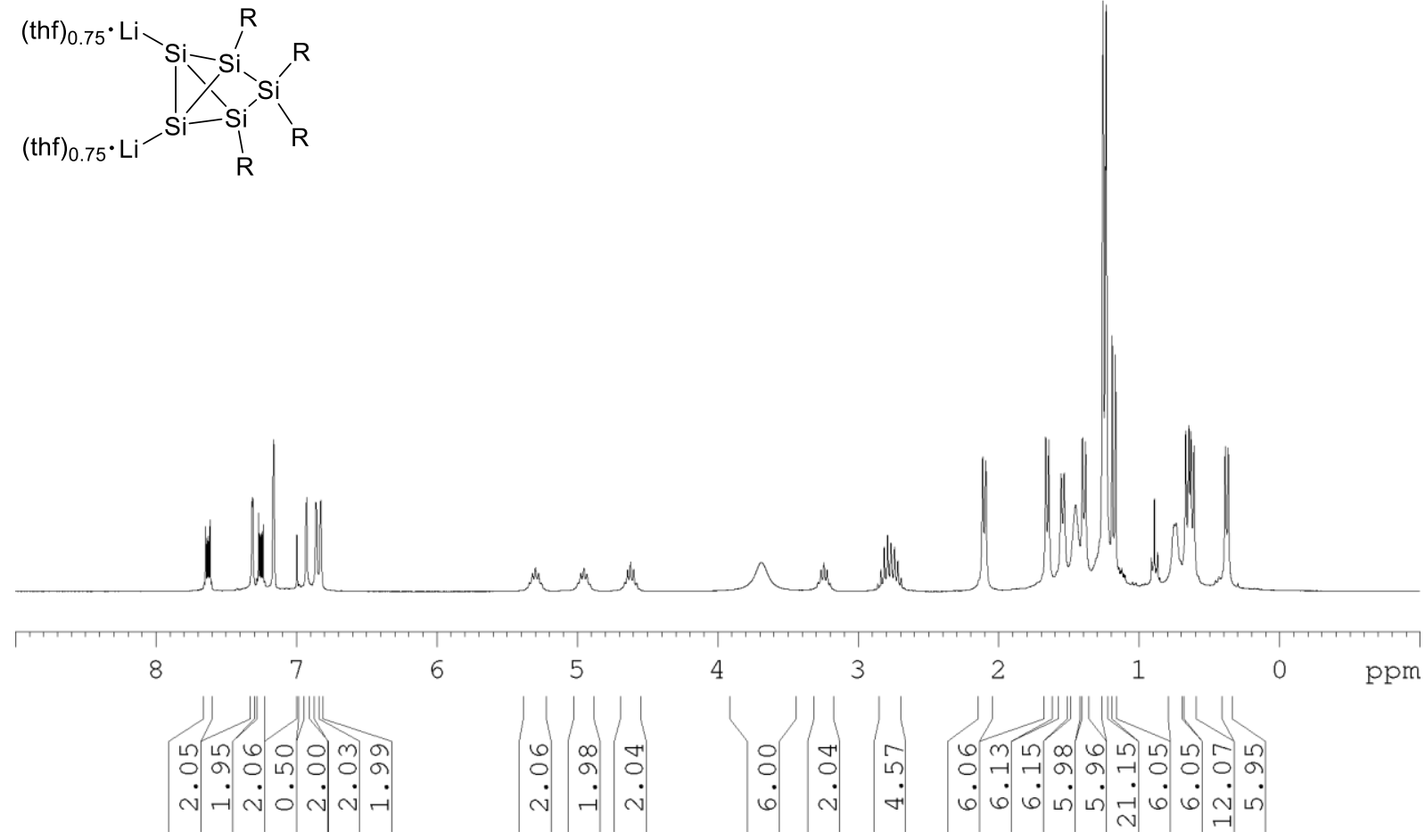

Supplementary Figure 1: ${ }^{1} \mathrm{H}$ NMR spectrum of 4 in $\mathrm{C}_{6} \mathrm{D}_{6}$ at $300 \mathrm{~K}$. 
g.,.

เก เ

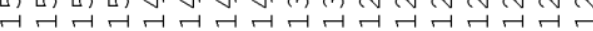

$\infty$ ๘

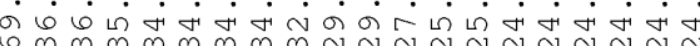
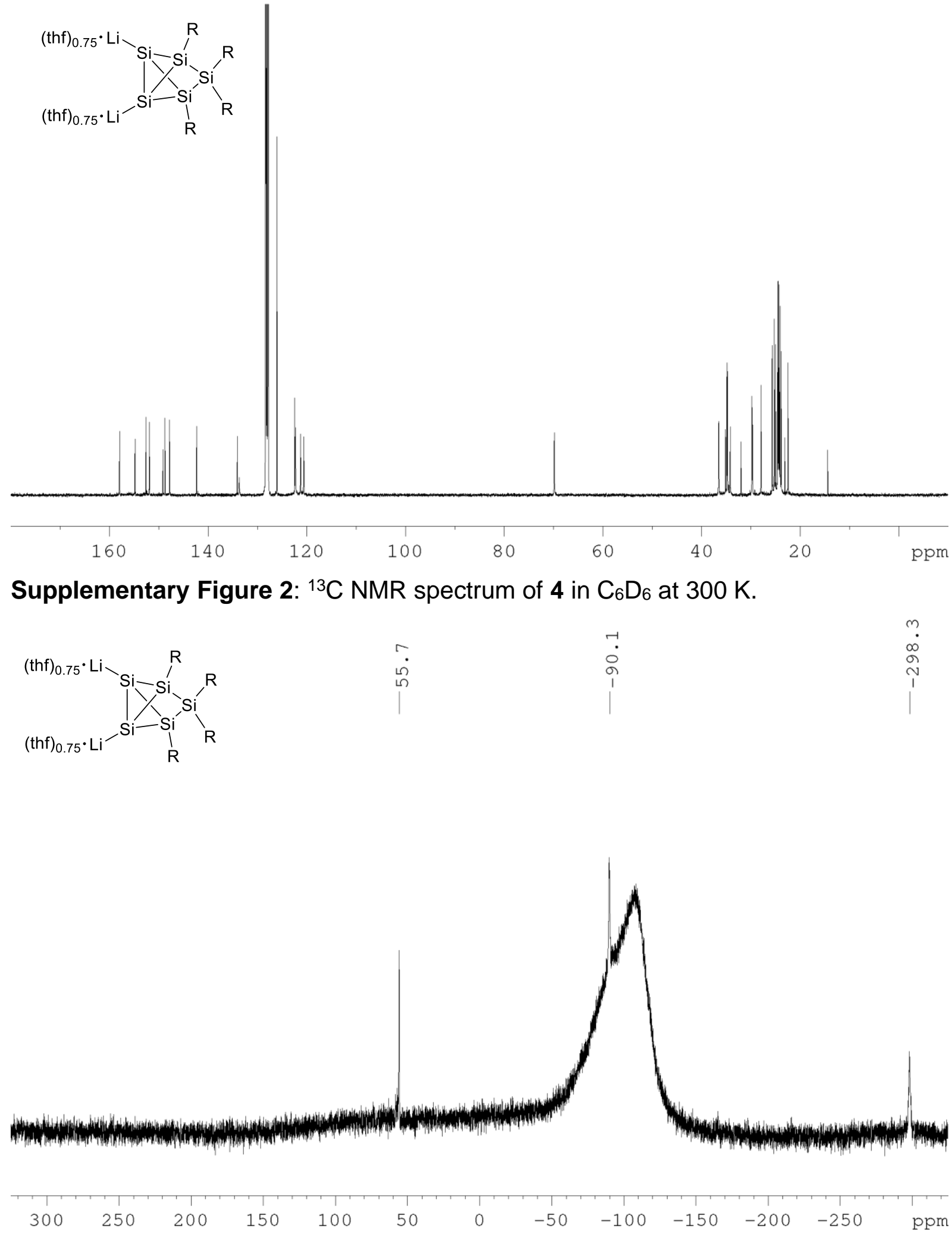

Supplementary Figure 3: ${ }^{29} \mathrm{Si}$ NMR spectrum of 4 in $\mathrm{C}_{6} \mathrm{D}_{6}$ at $300 \mathrm{~K}$. 

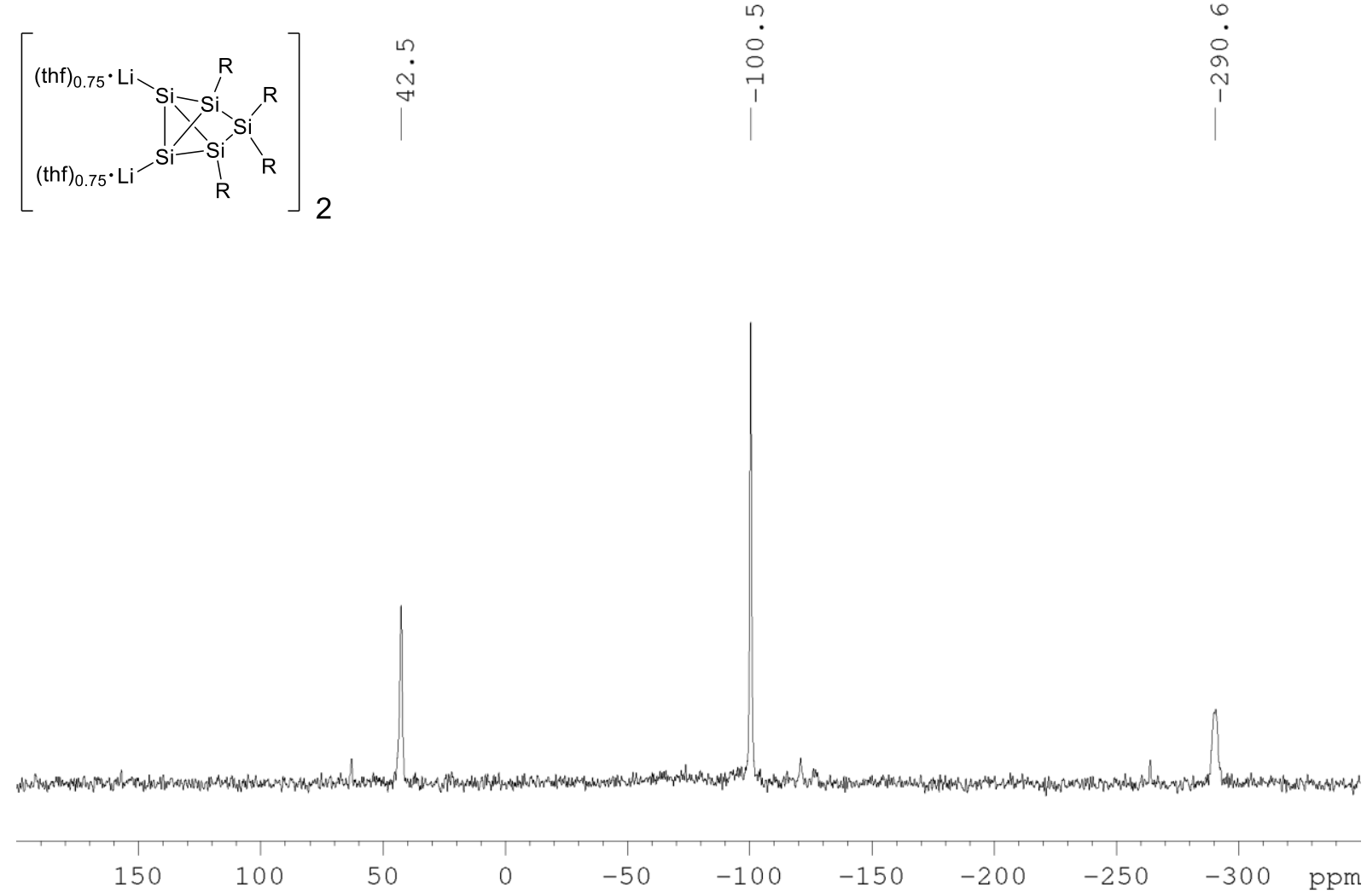

Supplementary Figure $4:{ }^{29} \mathrm{Si} \mathrm{CP/MAS} \mathrm{NMR} \mathrm{spectrum} \mathrm{of}[4]_{2} \cdot(\mathrm{THF})_{3}$.
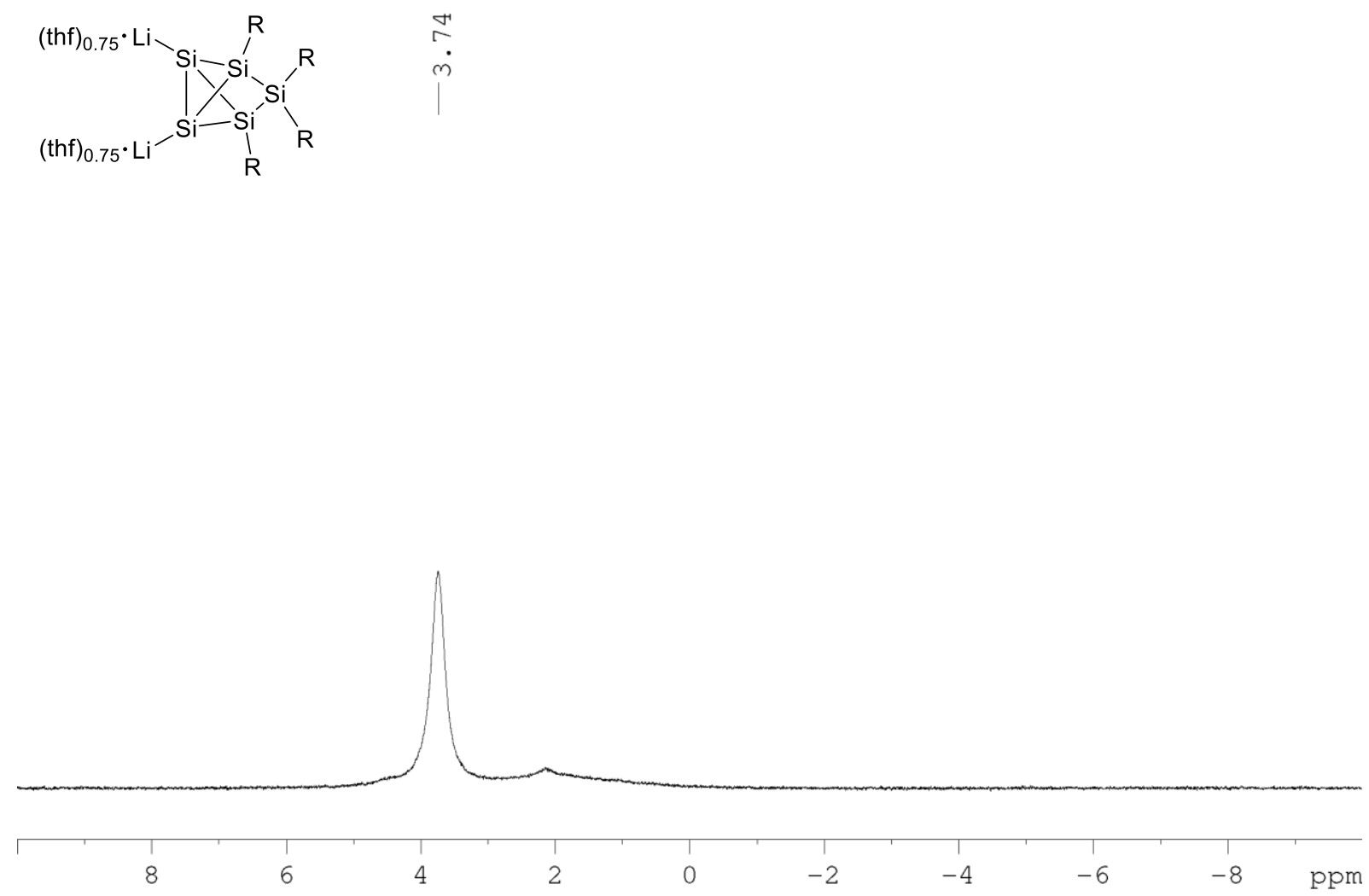

Supplementary Figure 5: ${ }^{7} \mathrm{Li}$ NMR spectrum of 4 in $\mathrm{C}_{6} \mathrm{D}_{6}$ at $300 \mathrm{~K}$. 

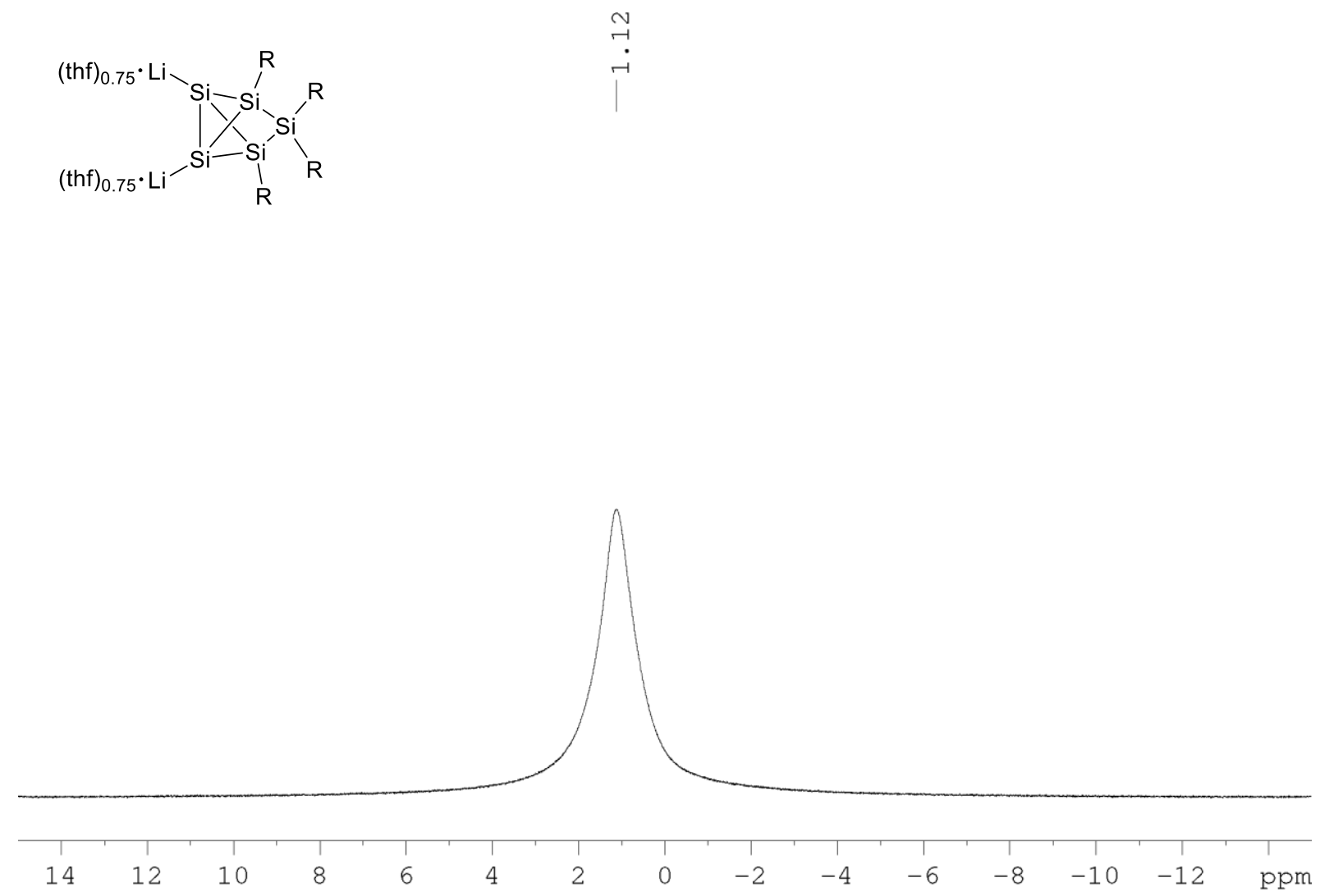

Supplementary Figure 6: ${ }^{7} \mathrm{Li}$ NMR spectrum of 4 in thf-d8 at $300 \mathrm{~K}$.
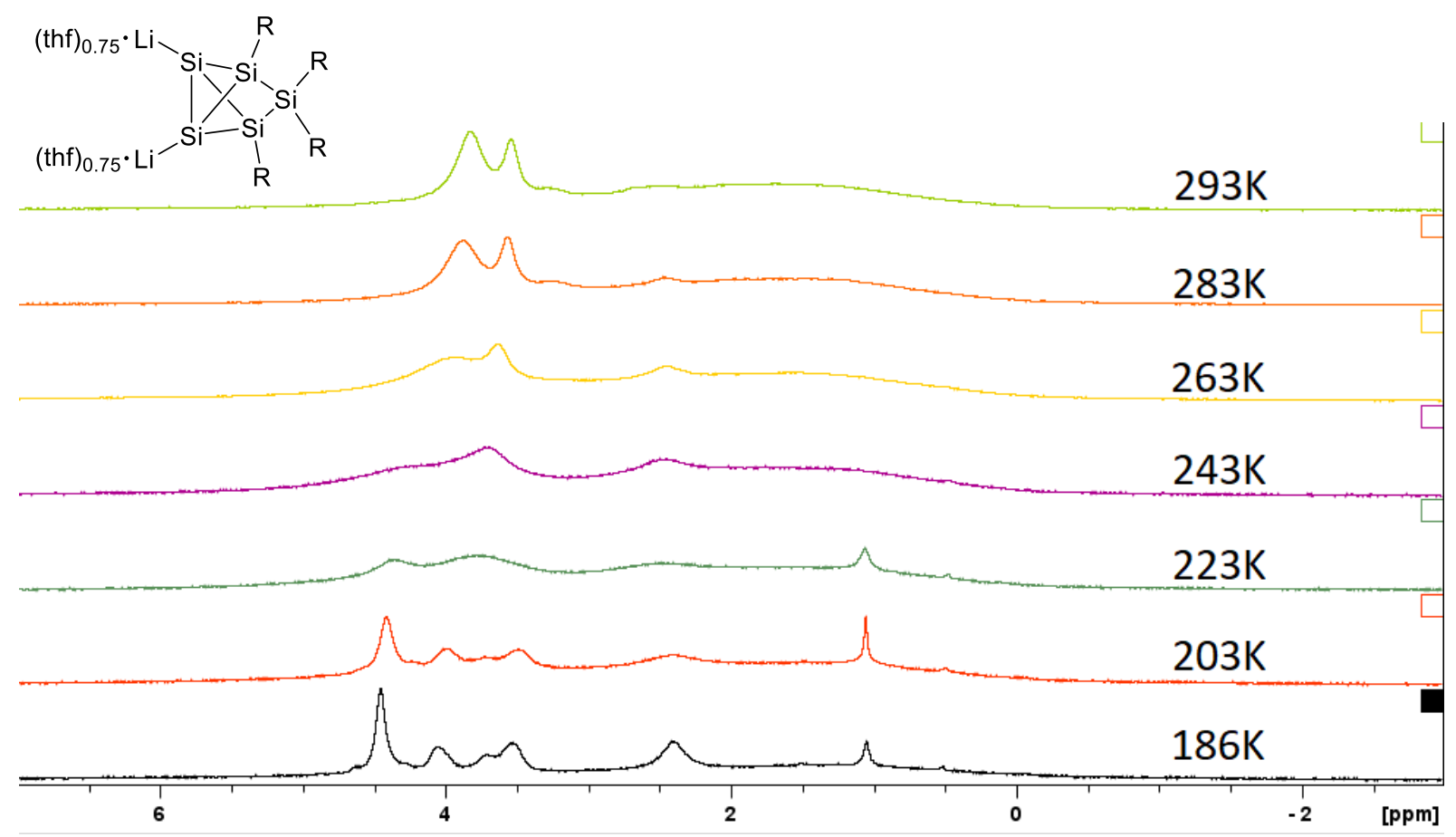

Supplementary Figure 7: ${ }^{7} \mathrm{Li}$ NMR spectra of $\mathbf{4}$ in toluene-d8 at different temperatures $(186 \mathrm{~K}-293 \mathrm{~K})$. 

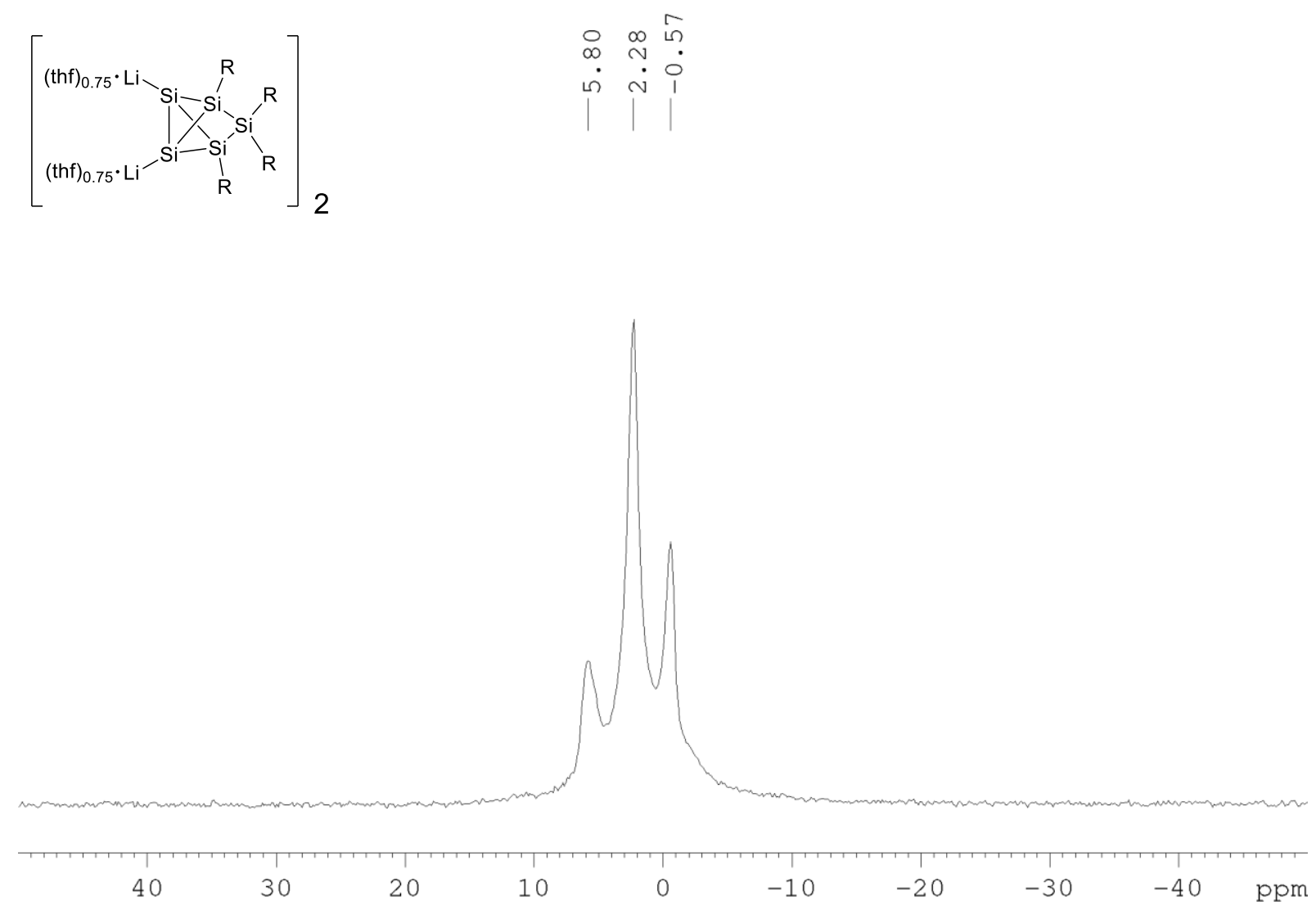

Supplementary Figure 8: ${ }^{7} \mathrm{Li}\left\{{ }^{1} \mathrm{H}\right\} \mathrm{CP} / \mathrm{MAS}$ NMR spectrum of $[4]_{2} \cdot(\mathrm{THF})_{3}$.

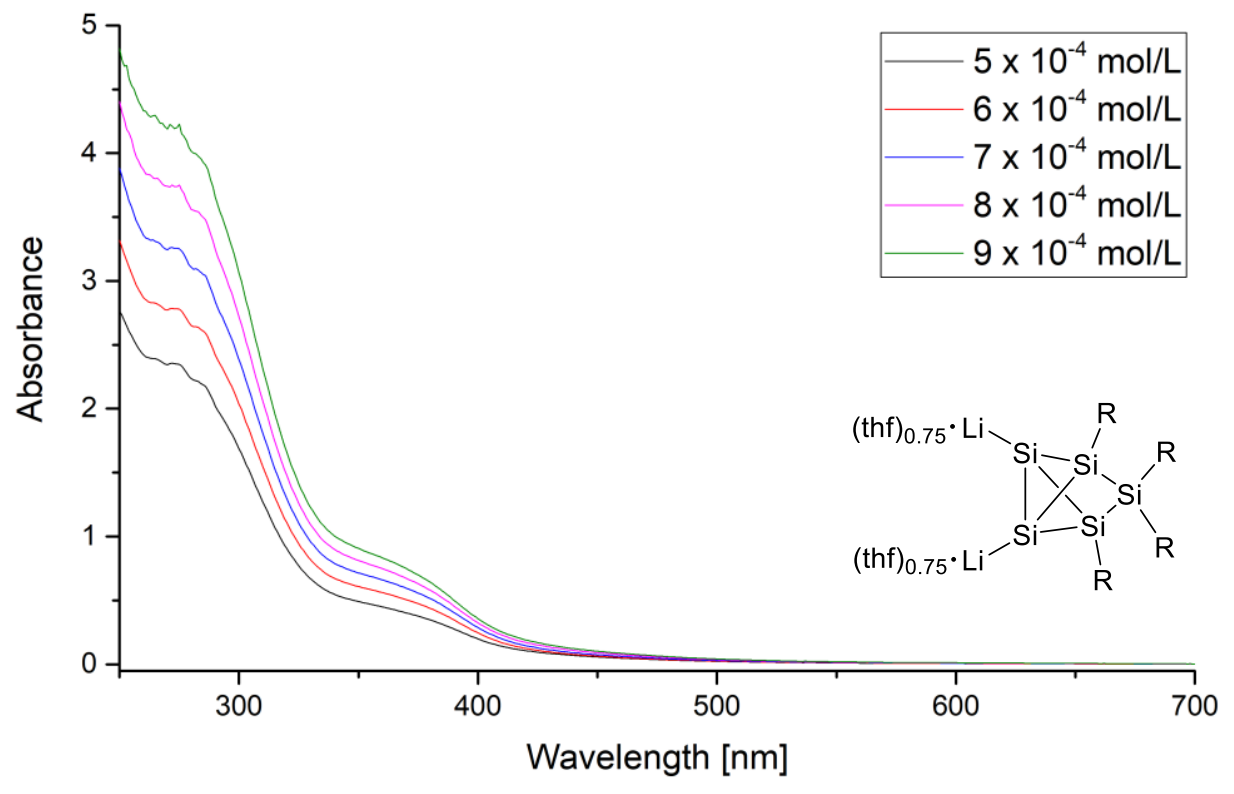

Supplementary Figure 9: UV/Vis spectra of 4 in hexane at different concentrations $\left(5 \cdot 10^{-4}-9 \cdot 10^{-4} \mathrm{~mol} / \mathrm{L}\right)$. 


\subsection{2,3,3,4-Tetrakis(2,4,6-triisopropylphenyl)-1,5- bis(trimethylsilyl)tricyclo[2.1.0.02,5]pentasilane (7)}

$230 \mathrm{mg}(0.20 \mathrm{mmol})$ of dianionic Sis-cluster 4 are dissolved in $2.5 \mathrm{~mL}$ of benzene. 51 $\mu \mathrm{L}(0.40 \mathrm{mmol})$ of $\mathrm{Me}_{3} \mathrm{SiCl}$ are added at room temperature. The resulting yellowbrownish reaction mixture is stirred for 30 minutes before all volatiles are removed under reduced pressure. The solid residue is digested with hexane and filtered. The filtrate is reduced to dryness in vacuo. Crystallization from pentane at $-26^{\circ} \mathrm{C}$ afforded $59 \mathrm{mg}(27 \%)$ of the Bis(trimethylsilyl)substituted Sis-cluster $\mathbf{7}$ as yellow crystals. (m.p. $>220^{\circ} \mathrm{C}$, dec.)

${ }^{1} \mathrm{H}$ NMR $\left(400.13 \mathrm{MHz}, \mathrm{C}_{6} \mathrm{D}_{6}, 300 \mathrm{~K}\right): \delta=7.26,7.12,7.00,6.81$ (each d, each $2 \mathrm{H}$, TipH), 4.92, 4.74, 3.78, 3.04 (each sept, each 2H, Tip-Pr-CH), 2.77 to 2.67 (m, 4H, Tip(Pr-CH) , 2.07, 1.62, 1.57, 1.43, 1.38 (each d, each 6H, Tip-Pr-CH $\left.\mathrm{CH}_{3}\right) 1.28$ to $1.22(\mathrm{~m}$, pentane), 1.20, 1.20 (overlapping $\mathrm{d}$, each $6 \mathrm{H}$, Tip-PPr- $\mathrm{CH}_{3}$ ) 1.14, 1.13 (overlapping $\mathrm{d}$, each 6H, Tip- $-\mathrm{Pr}-\mathrm{CH}_{3}$ ), 0.88 (t, pentane), 0.64, 0.58 (each d, each 6H, Tip-iPr-CH $\mathrm{CH}_{3}$ ), $0.46\left(\mathrm{~s}, 18 \mathrm{H}, \mathrm{Si}\left(\mathrm{CH}_{3}\right)_{3}\right), 0.23$ (d, 6H, Tip- $\left.\mathrm{Pr}-\mathrm{CH}_{3}\right)$.

${ }^{13} \mathrm{C}$ NMR $\left(100.6 \mathrm{MHz}, \mathrm{C}_{6} \mathrm{D}_{6}, 300 \mathrm{~K}\right): \delta=158.2,156.4,153.5,152.6,150.8,149.1$, 138.4, 126.3 (Tip-Ar-C), 122.9, 122.8, 121.8, 120.9 (Tip-Ar-CH), 38.0, 37.6, 36.9, 34.8, 34.7, 34.7 (Tip-Pr- $C H$ ), 34.5 (pentane), 29.5, 29.2, 27.6, 24.8, 24.7, 24.5, 24.2, 24.0, 23.8 (Tip- $-\mathrm{Pr}-\mathrm{CH}_{3}$ ), 22.7 (pentane), 22.6 (Tip- $-\mathrm{Pr}-\mathrm{CH}_{3}$ ), 14.3 (pentane), 5.5 $\left(\mathrm{Si}\left(\mathrm{CH}_{3}\right)_{3}\right.$.

${ }^{29} \mathrm{Si} \mathrm{NMR}\left(79.5 \mathrm{MHz}, \mathrm{C}_{6} \mathrm{D}_{6}, 300 \mathrm{~K}\right): \delta=34.7$ (SITip $), 1.7\left(\mathrm{Si}\left(\mathrm{CH}_{3}\right)_{3}\right),-81.3$ (SiTip), $-231.3(S i)$.

UV/Vis (hexane): broad shoulders from $\lambda_{\max }=320-390 \mathrm{~nm}$ and $\lambda_{\max }=260-285$ $\mathrm{nm}$.

Elemental analysis: calculated for $\mathrm{C}_{66} \mathrm{H}_{110} \mathrm{Si}_{77} \mathrm{C}, 72.05 \% ; \mathrm{H}, 10.08 \% ; \mathrm{N}, 0.00 \%$. Found: C, 73.89; H, 9.58; N, 1.38. 

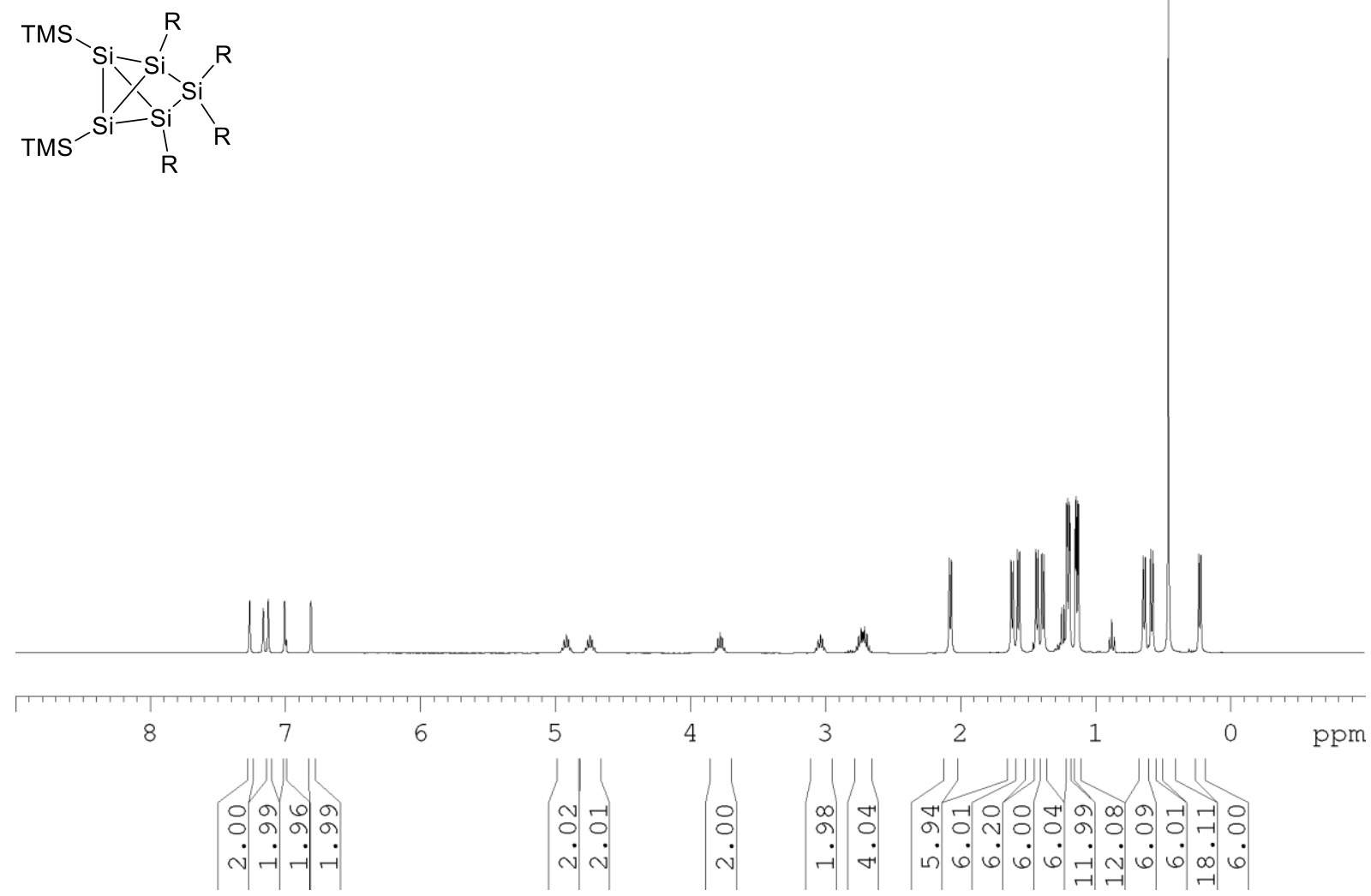

Supplementary Figure 10: ${ }^{1} \mathrm{H}$ NMR spectrum of 7 in $\mathrm{C}_{6} \mathrm{D}_{6}$ at $300 \mathrm{~K}$.

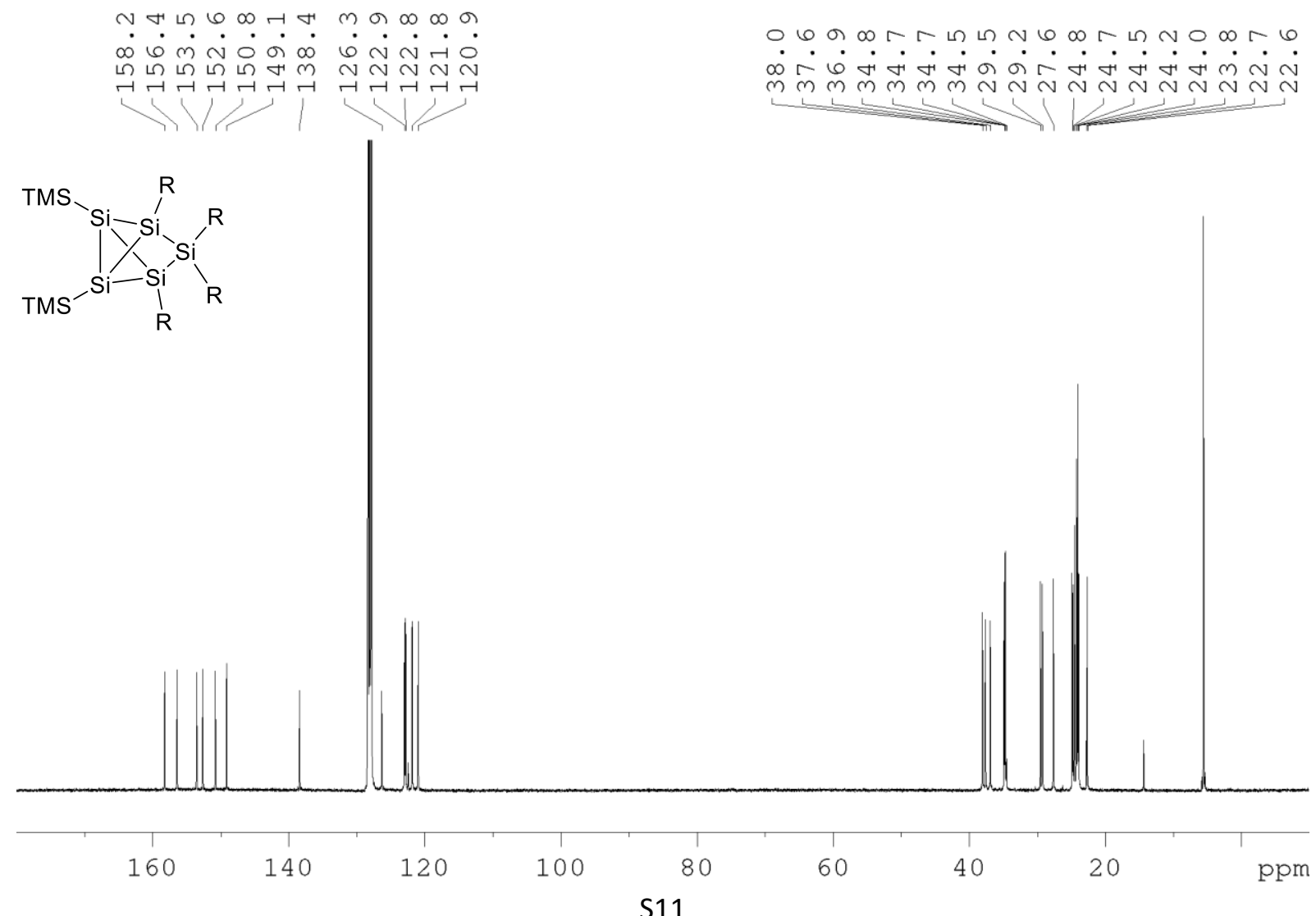


Supplementary Figure $11:{ }^{13} \mathrm{C}$ NMR spectrum of 7 in $\mathrm{C}_{6} \mathrm{D}_{6}$ at $300 \mathrm{~K}$.
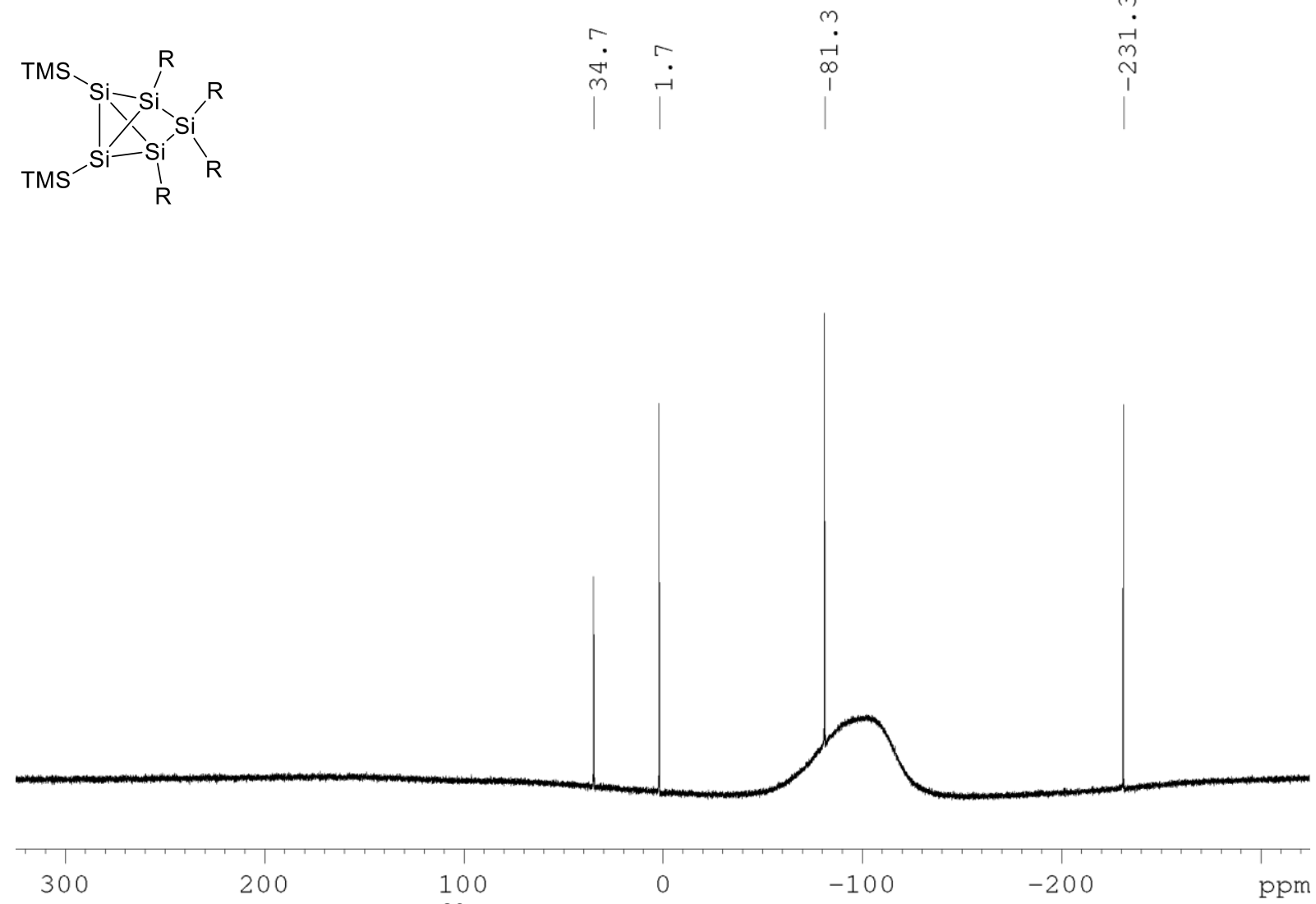

Supplementary Figure 12: ${ }^{29} \mathrm{Si}$ NMR spectrum of 7 in $\mathrm{C}_{6} \mathrm{D}_{6}$ at $300 \mathrm{~K}$.

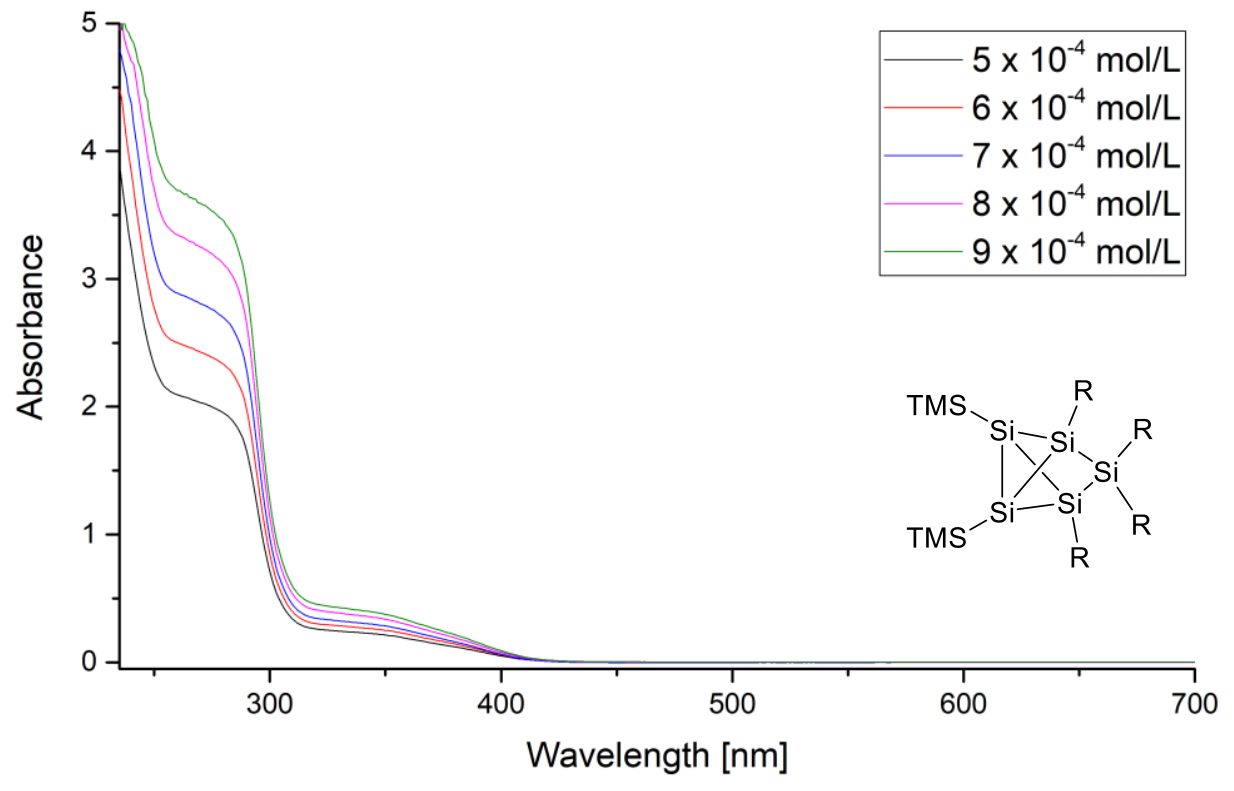

Supplementary Figure 13: UV/Vis spectra of 7 in hexane at different concentrations $\left(5 \cdot 10^{-4}-9 \cdot 10^{-4} \mathrm{~mol} / \mathrm{L}\right)$. 


\subsection{N,N-Diisopropyl-4,5,5,6-tetrakis(2,4,6-triisopropylphenyl)-1,3,4,5,6- pentasila-2-boratetracyclo[2.2.0.0 $\left.0^{1,3} \cdot 0^{3,6}\right]$ hexan-2-amine (8)}

$249 \mathrm{mg}(0.21 \mathrm{mmol})$ of dianionic Sis-cluster 4 are dissolved in $2.5 \mathrm{~mL}$ of benzene. 40 $\mu \mathrm{L}(0.23 \mathrm{mmol})$ of dichloro(diisopropylamino)borane are added at r.t. and the resulting reaction mixture is stirred for 30 minutes. All volatiles are removed in vacuo. The solid residue is digested with hexane and filtered. Crystallization from hexane at r.t. yields $132 \mathrm{mg}(59 \%)$ of $\mathrm{BSi}_{5}$ cluster 8 as orange crystals (m.p. $>220^{\circ} \mathrm{C}$, stable). ${ }^{1} \mathrm{H}$ NMR $\left(300.13 \mathrm{MHz}, \mathrm{C}_{6} \mathrm{D}_{6}, 300 \mathrm{~K}\right): \delta=7.25,7.11,6.99,6.83$ (each d, each $2 \mathrm{H}$, TipH), $5.26,4.66,4.14$ (each sept, each 2H, Tip-Pr-CH), 3.43 (sept, 2H, B-N-Pr-CH), 3.17 (sept, 2H, Tip-IPr-CH), 2.84 to 2.63 (m, 4H, Tip-Pr-CH), 2.10, 1.66, 1.57, 1.53 (each d, each 6H, Tip-Pr- $\mathrm{CH}_{3}$ ), 1.25 to 1.21 (m, 18H, Tip-Pr-CH $\mathrm{CH}_{3}, 1.10$ (dd, $12 \mathrm{H}$, Tip-Pr- $\mathrm{CH}_{3}$ ), 0.95, 0.87 (each d, each 6H, B-N-Pr-CH3), 0.71, 0.62, 0.31 (each d, each $6 \mathrm{H}$, Tip- $\left.-\mathrm{Pr}-\mathrm{CH}_{3}\right)$.

${ }^{13} \mathrm{C}$ NMR $\left(75.5 \mathrm{MHz}, \mathrm{C}_{6} \mathrm{D}_{6}, 300 \mathrm{~K}\right): \delta=157.7,155.8,153.3,152.1,150.3,149.2$, 139.5, 128.0, 123.0, 122.5, 121.3, 120.4 (Tip- $C$ and Tip- $C H$ ), 53.9 (B-N-Pr-CH), 37.3, 36.5, 36.0, 35.1, 34.7 (Tip-'Pr- $\mathrm{CH}$ and Tip-PPr- $\mathrm{CH}_{3}$ ) 32.0 (hexane), 30.1, 27.8, 27.3, 24.7, 24.5, 24.2, 24.2, 24.1, 24.0, 24.0,23.5 (Tip-'Pr-CH and Tip- ${ }^{-} \mathrm{Pr}-\mathrm{CH}_{3}$ ), 23.4 (B-N-Pr- $\mathrm{CH}_{3}$ ), 23.06 (hexane), 21.4 (Tip- $-\mathrm{Pr}-\mathrm{CH}_{3}$ ), 14.4 (hexane).

${ }^{29} \mathrm{Si} \mathrm{NMR}\left(59.6 \mathrm{MHz}, \mathrm{C}_{6} \mathrm{D}_{6}, 300 \mathrm{~K}\right): \delta=39.0$ (SiTip 2 ), -43.4 (SITip), -288.6 (SI).

${ }^{11} \mathrm{~B}$ NMR (128.4 MHz, $\left.\mathrm{C}_{6} \mathrm{D}_{6}, 300 \mathrm{~K}\right): \delta=83.3$.

UV/Vis (hexane): $\lambda_{\max }=291 \mathrm{~nm}\left(\varepsilon=37820 \mathrm{M}^{-1} \mathrm{~cm}^{-1}\right) ; \lambda_{\max }=472 \mathrm{~nm}$ (very unintense).

Elemental analysis: calculated for $\mathrm{C}_{66} \mathrm{H}_{106} \mathrm{BNSi}_{5}$ : C, $74.45 \% ; \mathrm{H}, 10.03 \%$; N, $1.32 \%$. Found: C, 73.89; $\mathrm{H}, 9.58 ; \mathrm{N}, 1.38$. 


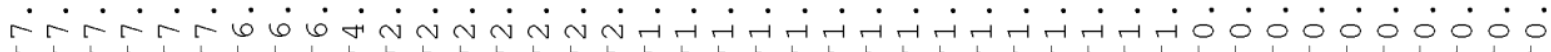
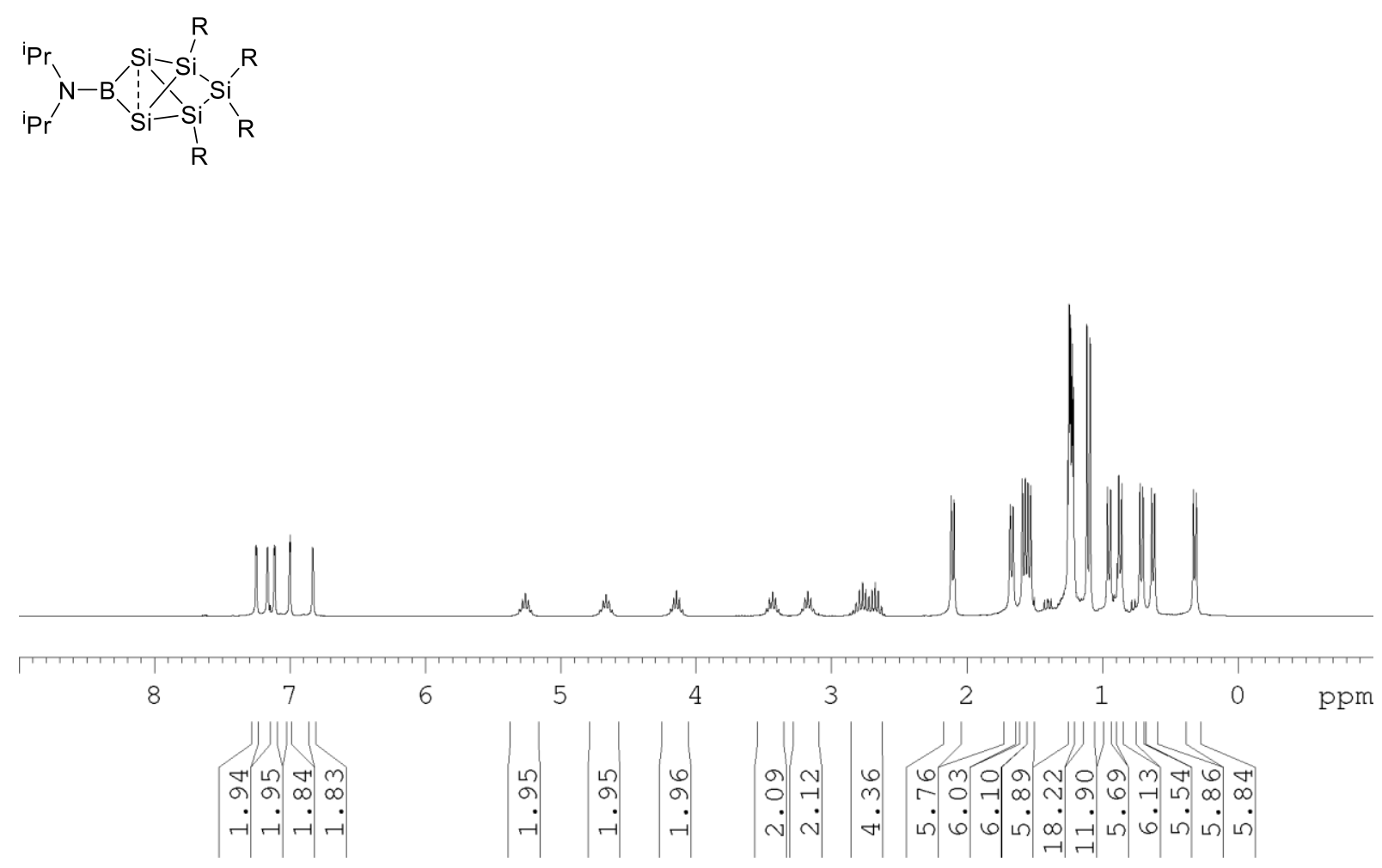

Supplementary Figure 14: ${ }^{1} \mathrm{H}$ NMR spectrum of 8 in $\mathrm{C}_{6} \mathrm{D}_{6}$ at $300 \mathrm{~K}$. 


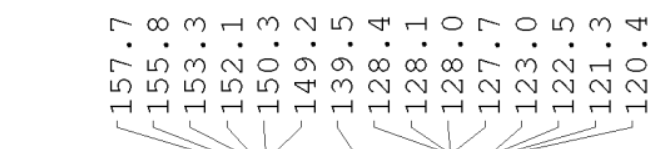

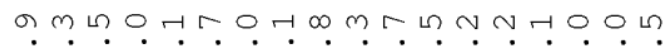

mं́ம்

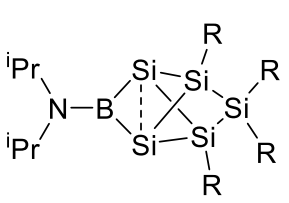

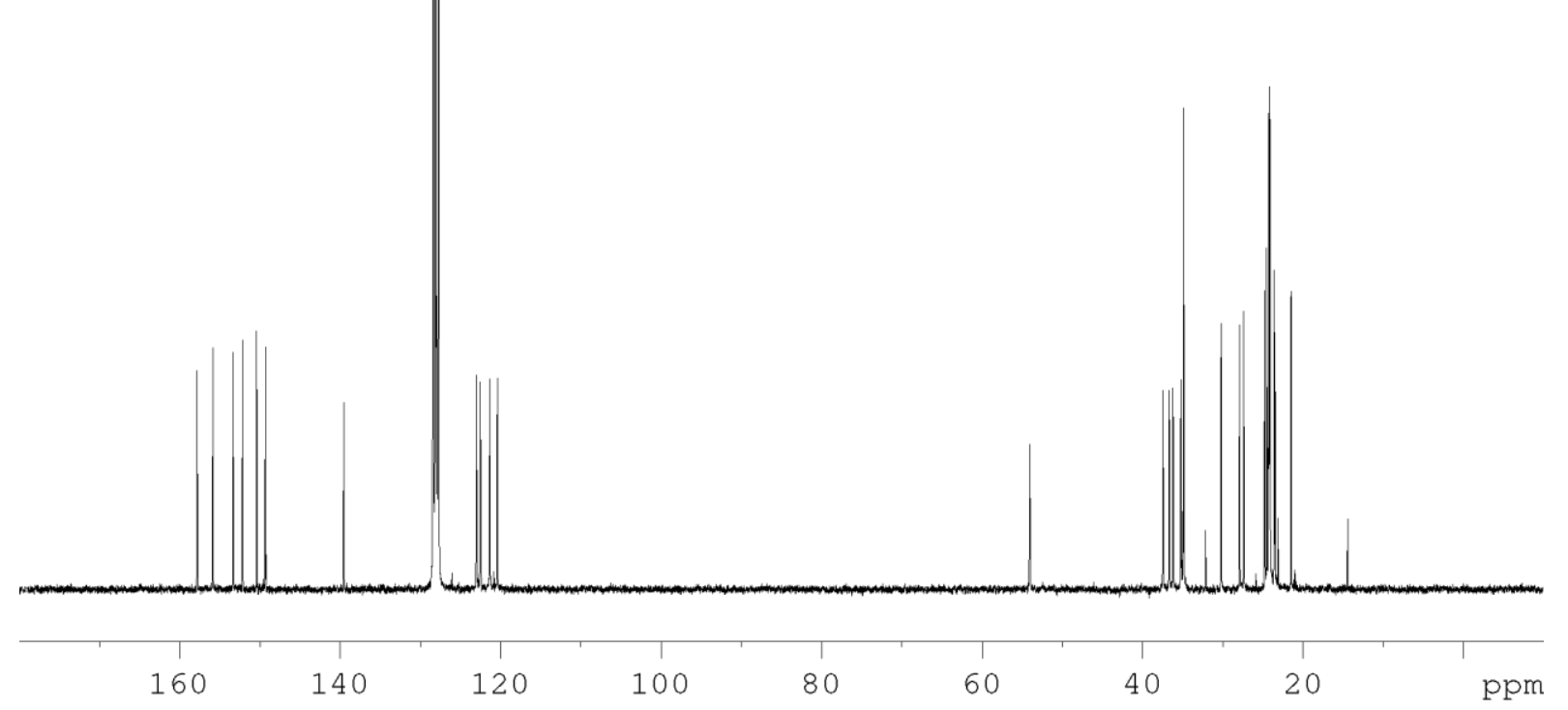

Supplementary Figure 15: ${ }^{13} \mathrm{C}$ NMR spectrum of 8 in $\mathrm{C}_{6} \mathrm{D}_{6}$ at $300 \mathrm{~K}$.

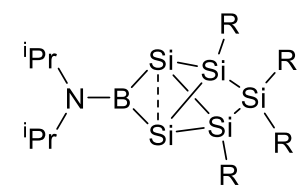

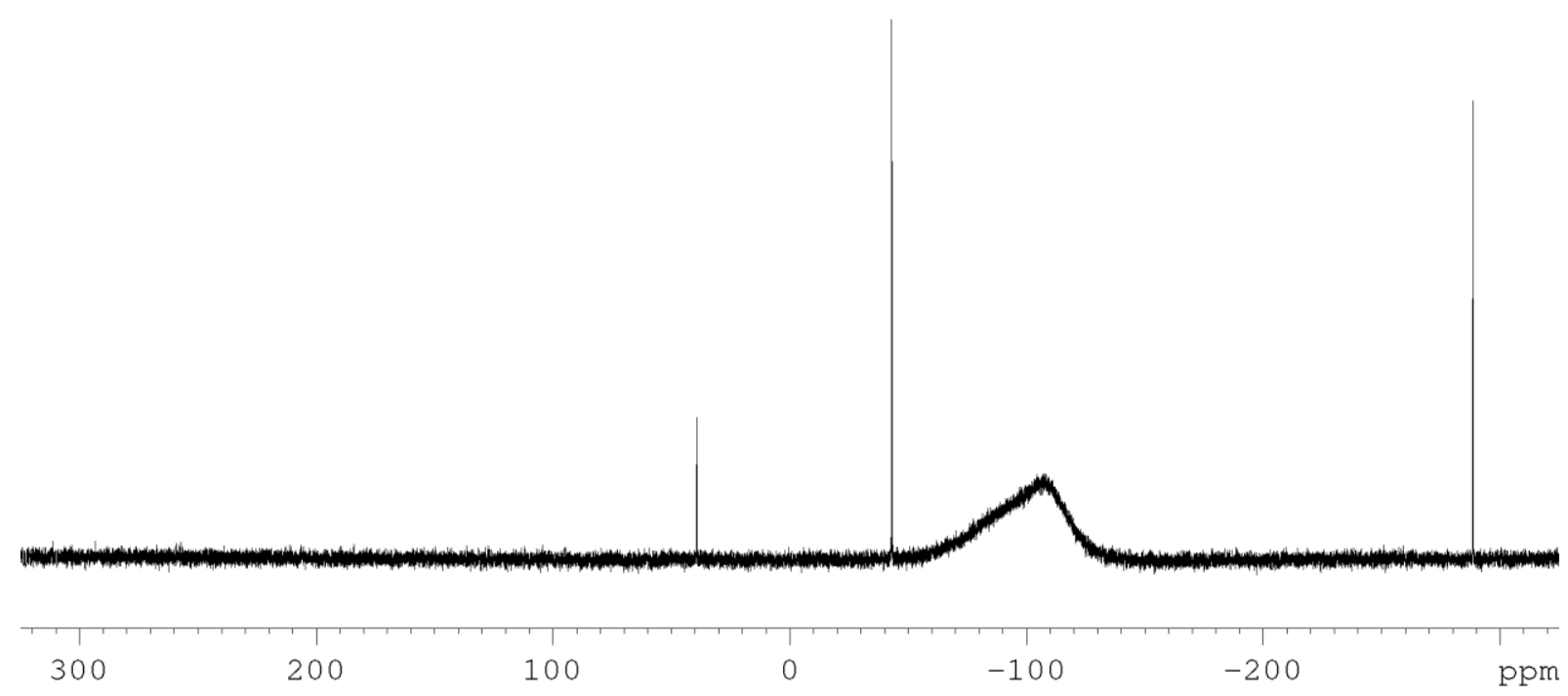

Supplementary Figure 16: ${ }^{29} \mathrm{Si}$ NMR spectrum of 8 in $\mathrm{C}_{6} \mathrm{D}_{6}$ at $300 \mathrm{~K}$. 

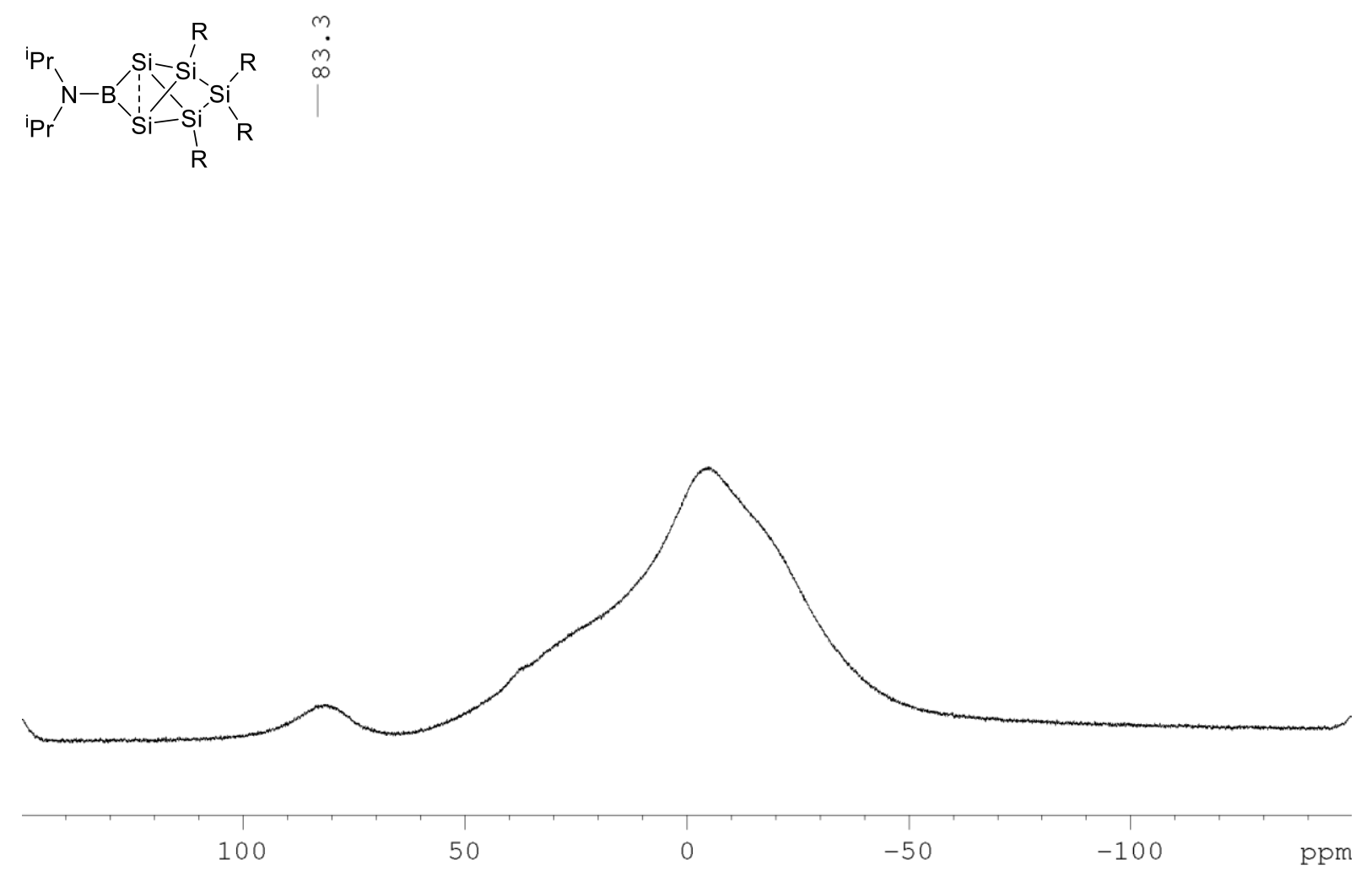

Supplementary Figure 17: ${ }^{11} \mathrm{~B}$ NMR spectrum of 8 in $\mathrm{C}_{6} \mathrm{D}_{6}$ at $300 \mathrm{~K}$.

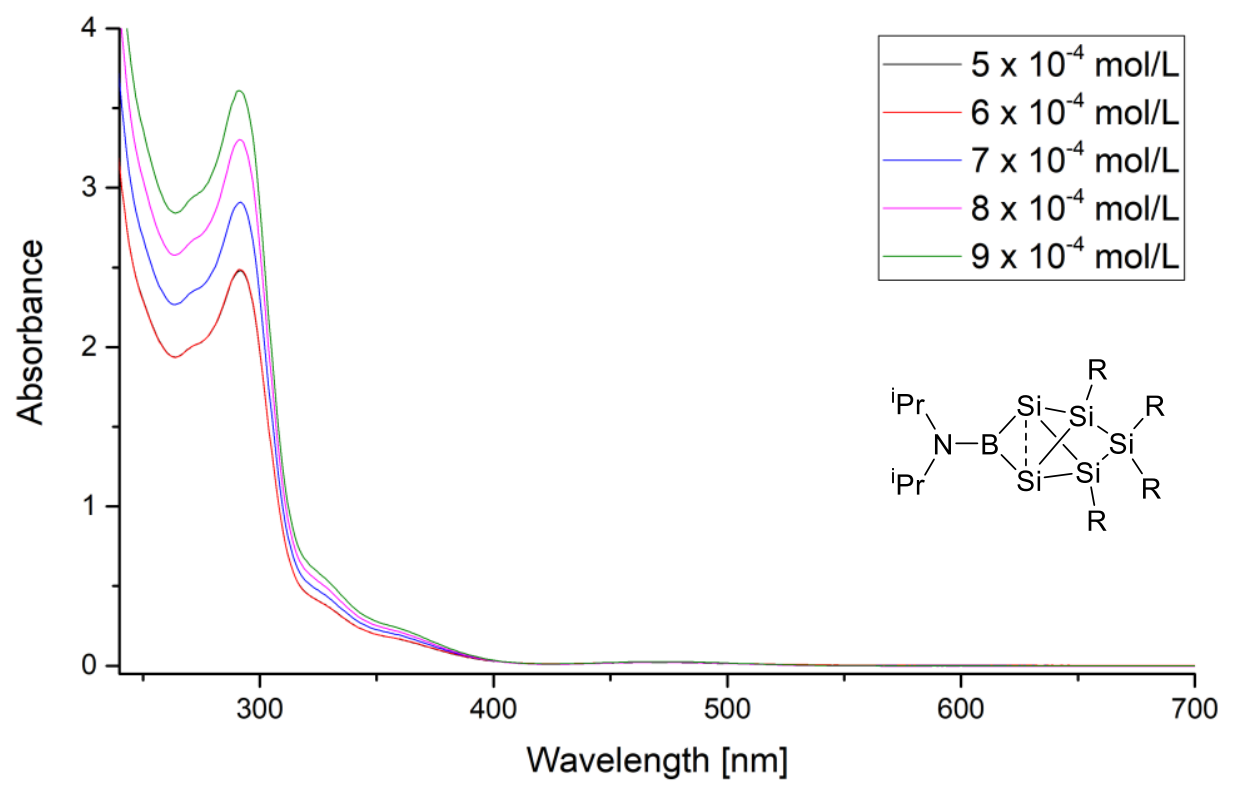

Supplementary Figure 18: UV/Vis spectra of 8 in hexane at different concentrations $\left(5 \cdot 10^{-4}-9 \cdot 10^{-4} \mathrm{~mol} / \mathrm{L}\right)$. 


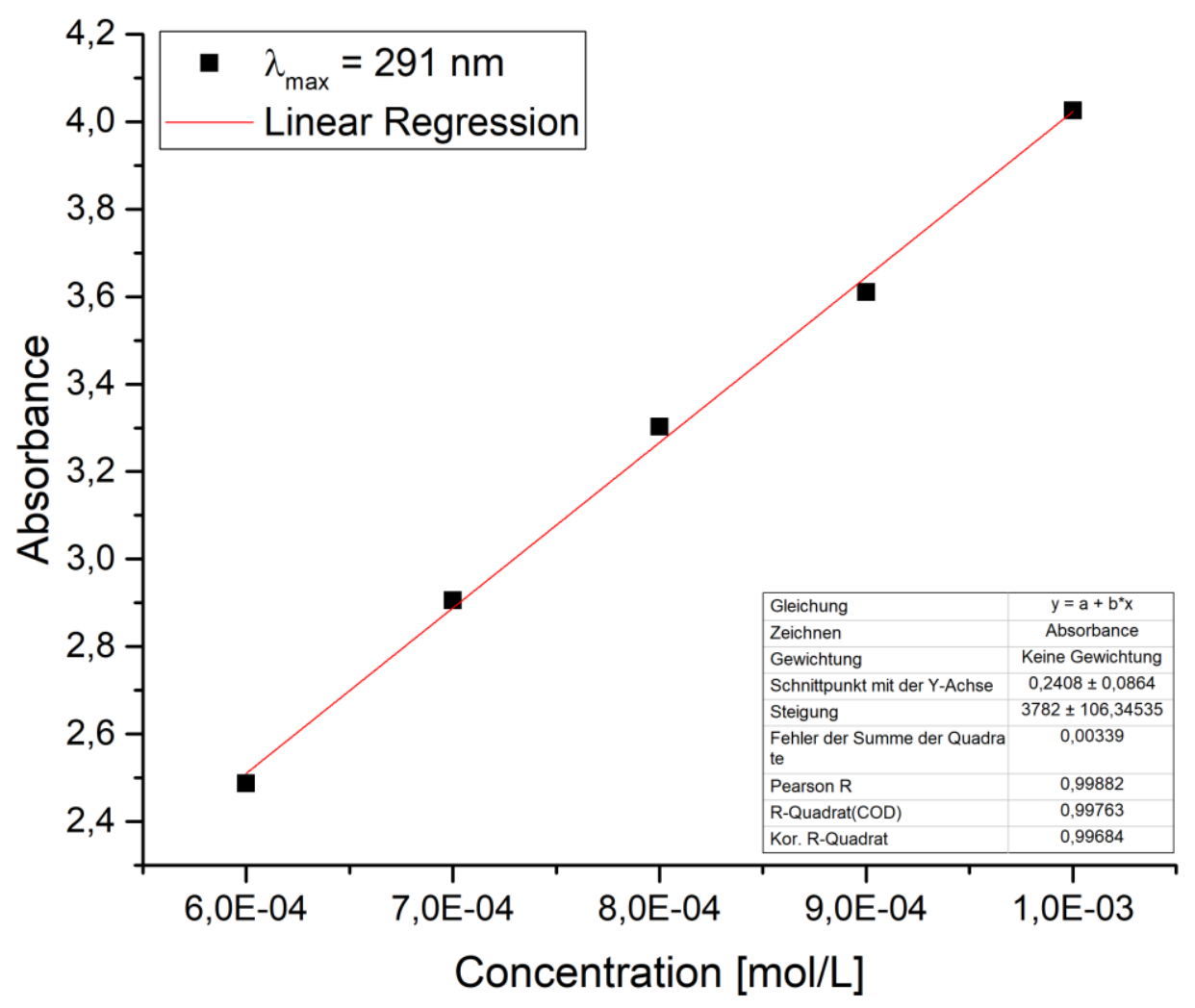

Supplementary Figure 19: Determination of extinction coefficient $\varepsilon\left(37820 \mathrm{M}^{-1} \mathrm{~cm}^{-1}\right)$ by linear regression of absorbance $(\lambda=291 \mathrm{~nm})$ of 8 against concentration.

\subsection{N,N-Diisopropyl-4,5,5,6-tetrakis(2,4,6-triisopropylphenyl)-2-phospha-}

\section{1,3,4,5,6-pentasilatetracyclo[2.2.0.0 $\left.0^{1,3} \cdot 0^{3,6}\right]$ hexan-2-amine (9)}

$257 \mathrm{mg}(0.21 \mathrm{mmol})$ of dianionic Sis-cluster 4 are dissolved in $4 \mathrm{~mL}$ of benzene.

Following $38 \mu \mathrm{L}(0.21 \mathrm{mmol})$ of dichloro(diisopropylamino)phosphine are added at r.t. and the resulting reaction mixture is stirred for 30 minutes. All volatiles are removed in vacuo. The solid residue is digested with hexane and filtered. Crystallization from hexane at $-26^{\circ} \mathrm{C}$ yields $89 \mathrm{mg}(42 \%)$ of PSis cluster 9 (m.p. $>220^{\circ} \mathrm{C}$, dec.). 
${ }^{1} \mathrm{H}$ NMR $\left(400.13 \mathrm{MHz}, \mathrm{C}_{6} \mathrm{D}_{6}, 300 \mathrm{~K}\right.$ ): $\delta=7.25,7.23,7.13,7.06$ (each d, each $1 \mathrm{H}$, TipH), 6.99 (s, 1,3,5-trisopropylbenzene), 6.97, 6.94, 6.85, 6.80 (each d, each $1 \mathrm{H}$, TipH), 5.18 (sept, $1 \mathrm{H}$, Tip-Pr-CH), 4.65 to 4.54 (m, 2H, Tip- $\mathrm{Pr}-\mathrm{CH}$ ), 4.06, 3.91, 3.46 (each sept, each 1H, Tip-iPr-CH), 3.40 to $3.33(\mathrm{~m}, 2 \mathrm{H}$, Tip-Pr- $\mathrm{CH}$ ), 2.83 to $2.65(\mathrm{~m}$, $6 \mathrm{H}$, overlapping septets of 1,3,5-trisopropylbenzene, Tip- $\mathrm{Pr}-\mathrm{CH}$ and $\mathrm{P}-\mathrm{N}-\mathrm{Pr}-\mathrm{CH}$ ), 2.09, 1.98 (each d, each 3H, Tip- $\mathrm{Pr}-\mathrm{CH}_{3}$ ), 1.63 to 1.56 (m, 18H, Tip- $\left.-\mathrm{Pr}-\mathrm{CH}_{3}\right), 1.50$, 1.42 (each d, each $3 \mathrm{H}$, Tip-iPr- $\mathrm{CH}_{3}$ ), 1.24 (d, 1,3,5-trisopropylbenzene), 1.22 to 1.18 (m, 12H, Tip-iPr-CH $\mathrm{CH}_{3}$ and hexane), 1.13 to $1.11\left(\mathrm{~m}, 12 \mathrm{H}\right.$, Tip- $\left.\mathrm{Pr}-\mathrm{CH}_{3}\right), 0.89(\mathrm{~m}, 3 \mathrm{H}$, Tip-Pr- $\mathrm{CH}_{3}$ and hexane), 0.83, 0.77 (each d, each 6H, P-N-Pr- $\left.\mathrm{CH}_{3}\right), 0.67$ to $0.63(\mathrm{~m}$, 9H, Tip-'Pr- $\mathrm{CH}_{3}$ ), 0.43 to 0.39 (m, 6H, Tip- $-\mathrm{Pr}-\mathrm{CH}_{3}$ ).

${ }^{13} \mathrm{C}$ NMR $\left(100.6 \mathrm{MHz}, \mathrm{C}_{6} \mathrm{D}_{6}, 300 \mathrm{~K}\right): \delta=157.9,156.9,156.5,155.0,154.0,153.5$, 152.5, 151.4, 151.0, 150.6, 149.5, 149.4, 149.1, 138.3, 136.8, 130.4, 125.1, 123.2, 123.1, 122.8, 122.5, 122.4 (Tip- $C$ and Tip- $C H$ ), 122.2 (1,3,5-trisopropylbenzene), 122.0, 121.5, 121.4 (Tip- $\mathrm{CH}$ ), 52.6 (br, P-N-iPr-CH), 37.9, 37.9, 37.6, 37.2, 36.8, 36.2, 35.5, 35.0, 34.8, 34.6, 34.6, 34.6 (Tip-'Pr-CH and Tip-iPr- $\mathrm{CH}_{3}$ ), 32.0 (hexane), 28.6, 28.5, 27.6, 27.5, 27.3, 26.8, 26.6, 25.6, 25.2, 25.0, 24.8, 24.6, 24.5, 24.2, 24.2, 24.2, 24.1, 24.0, 24.0, 23.5 (Tip-Pr-CH and Tip-Pr- $\mathrm{CH}_{3}$ ), 23.3 (br, P-N-Pr- $\mathrm{CH}_{3}$ ), 23.1 (hexane), 21.7 (br, $\left.\mathrm{P}-\mathrm{N}-\mathrm{Pr}-\mathrm{CH}_{3}\right), 14.4$ (hexane).

$\left.{ }^{29} \mathrm{Si} \mathrm{NMR} \mathrm{(79.5} \mathrm{MHz,} \mathrm{C}_{6} \mathrm{D}_{6}, 300 \mathrm{~K}\right): \delta=153.7$ (s, SiTip), 8.2 (d, $\left.{ }^{3} \mathrm{JSi}_{\mathrm{P}}=29.6 \mathrm{~Hz}, \mathrm{SiTip}_{2}\right)$ -76.7 (d, ${ }^{2} \mathrm{JSi}_{\mathrm{P}}=41.0 \mathrm{~Hz}$, SiTip), $-225.8\left(\mathrm{~d},{ }^{1} \mathrm{~J} \mathrm{Si}-\mathrm{P}=124.4 \mathrm{~Hz}, \mathrm{Si}\right),-240.0\left(\mathrm{~d},{ }^{1} \mathrm{JSi}_{\mathrm{P}} \mathrm{P}=\right.$ $105.4 \mathrm{~Hz}, \mathrm{Si})$.

${ }^{31}$ P NMR $\left(162.0 \mathrm{MHz}, \mathrm{C}_{6} \mathrm{D}_{6}, 300 \mathrm{~K}\right): \delta=386.7$.

UV/Vis (hexane): broad plateau at $\lambda_{\max }=250-265 \mathrm{~nm}\left(\varepsilon=40930 \mathrm{M}^{-1} \mathrm{~cm}^{-1}\right) ; \lambda_{\max }=$ $355 \mathrm{~nm}\left(\varepsilon=2860 \mathrm{M}^{-1} \mathrm{~cm}^{-1}\right) ; \lambda_{\max }=412 \mathrm{~nm}\left(\varepsilon=5010 \mathrm{M}^{-1} \mathrm{~cm}^{-1}\right) ; \lambda \max =511 \mathrm{~nm}$ (very unintense).

Elemental analysis: calculated for $\mathrm{C}_{66} \mathrm{H}_{106} \mathrm{NPSi}$ : $\mathrm{C}, 73.06 \% ; \mathrm{H}, 9.85 \% ; \mathrm{N}, 1.29 \%$. Found: C, 72.74; H, 9.43; N, 1.22 . 


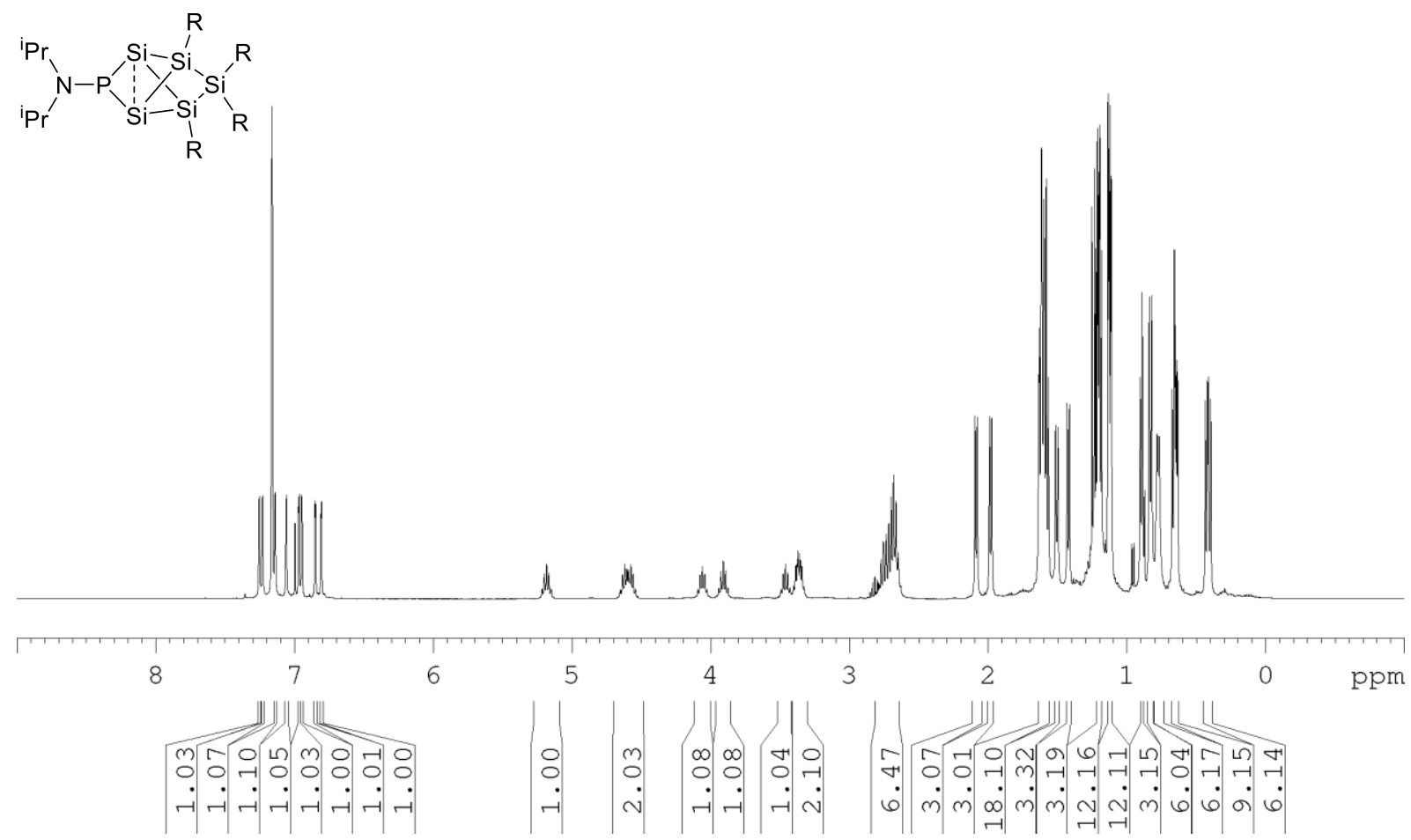

Supplementary Figure 20: ${ }^{1} \mathrm{H}$ NMR spectrum of 9 in $\mathrm{C}_{6} \mathrm{D}_{6}$ at $300 \mathrm{~K}$.

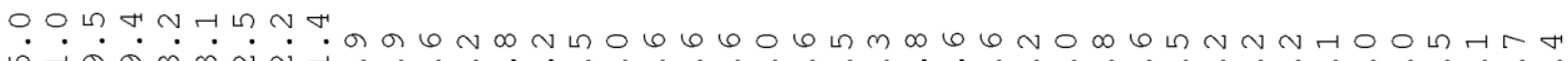
约 भी

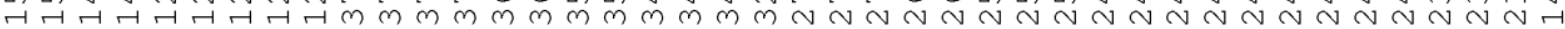

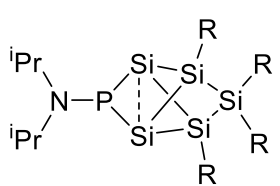

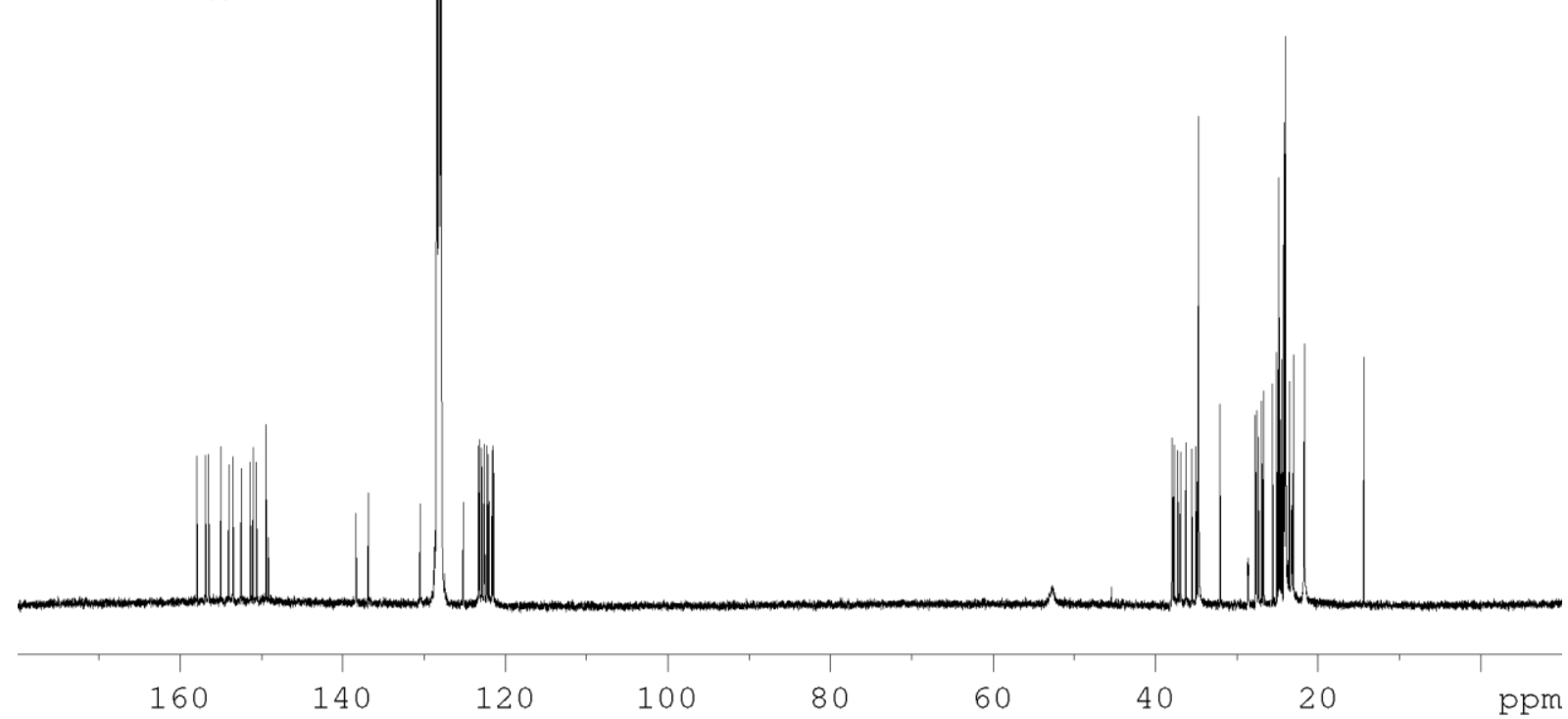

Supplementary Figure 21: ${ }^{13} \mathrm{C}$ NMR spectrum of 9 in $\mathrm{C}_{6} \mathrm{D}_{6}$ at $300 \mathrm{~K}$. 

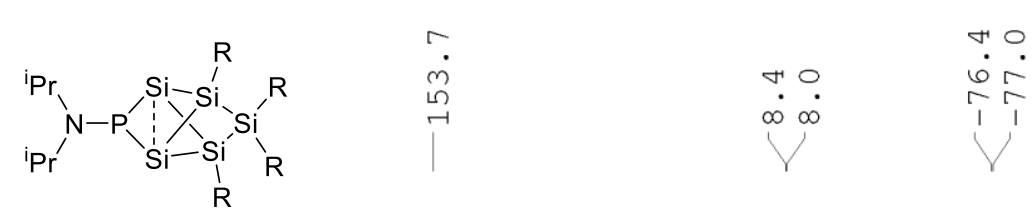

oLn H

เi $6 \dot{0}$

N $N \stackrel{\text { N }}{\sim}$ $\mathrm{R}$

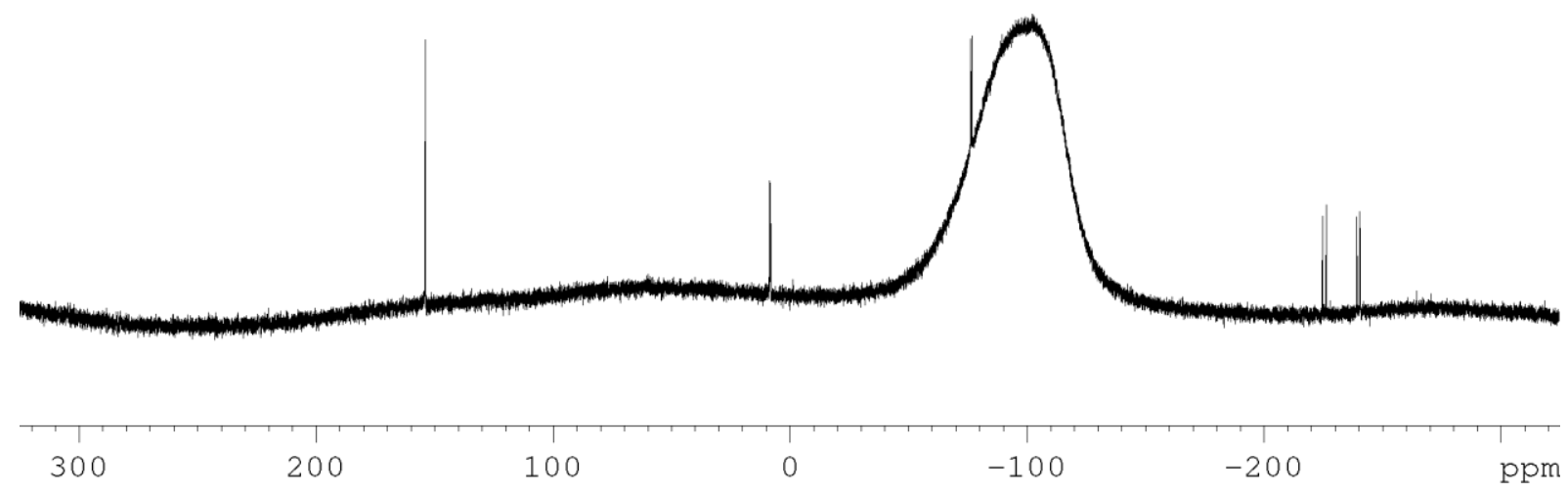

Supplementary Figure 22: ${ }^{29} \mathrm{Si}$ NMR spectrum of 9 in $\mathrm{C}_{6} \mathrm{D}_{6}$ at $300 \mathrm{~K}$.
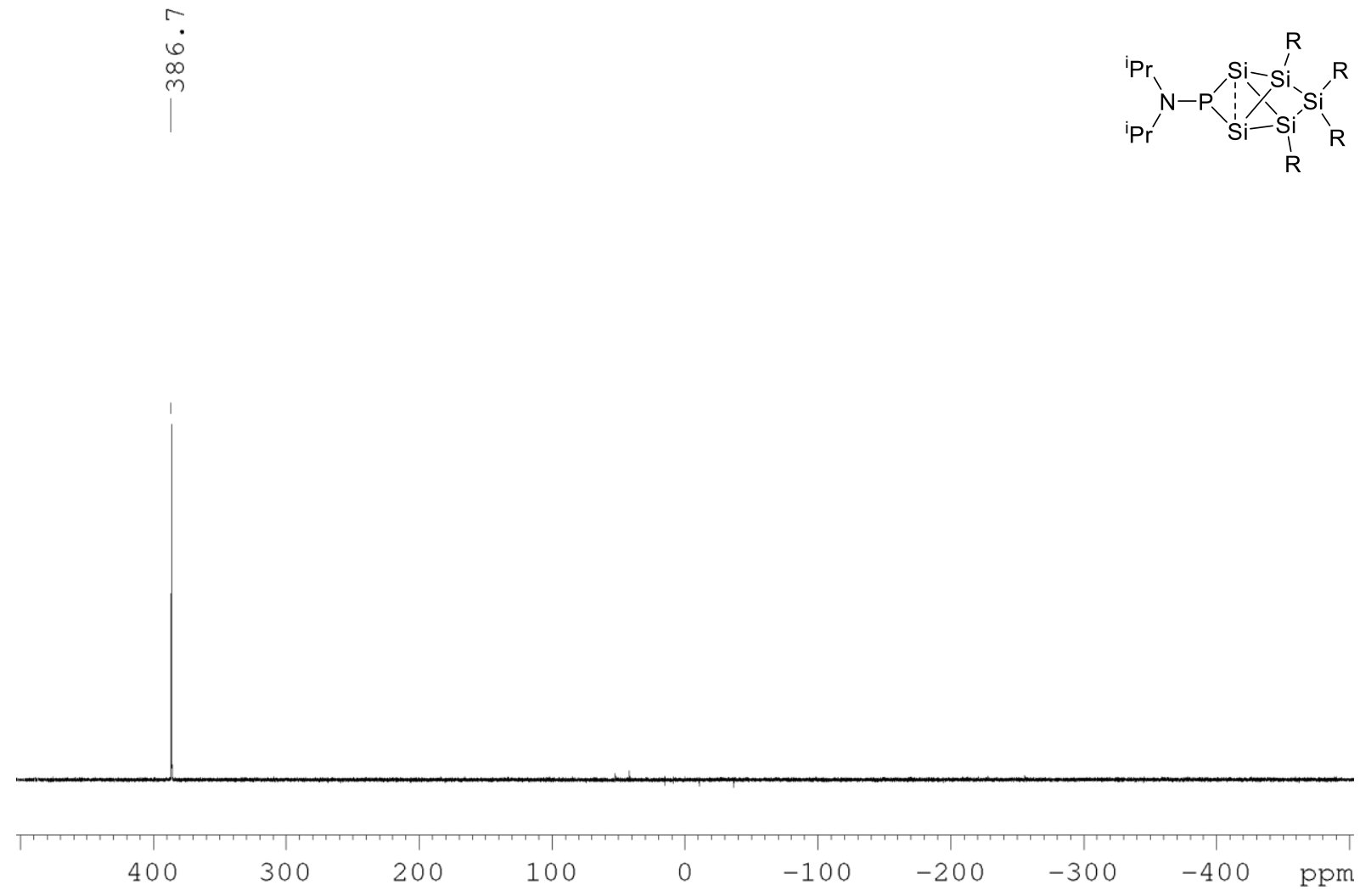

Supplementary Figure 23: ${ }^{31} \mathrm{P}$ NMR spectrum of 9 in $\mathrm{C}_{6} \mathrm{D}_{6}$ at $300 \mathrm{~K}$. 


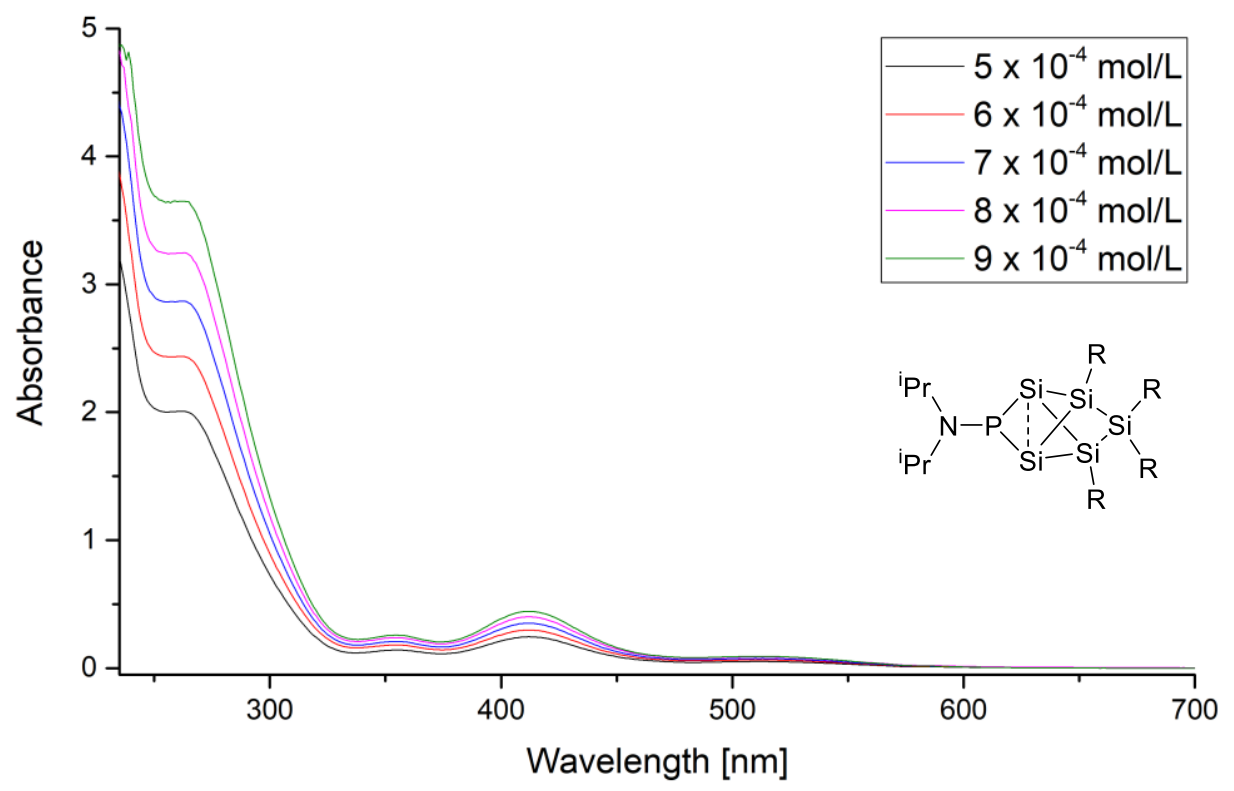

Supplementary Figure 24: UV/Vis spectra of 9 in hexane at different concentrations $\left(5 \cdot 10^{-4}-9 \cdot 10^{-4} \mathrm{~mol} / \mathrm{L}\right)$. 


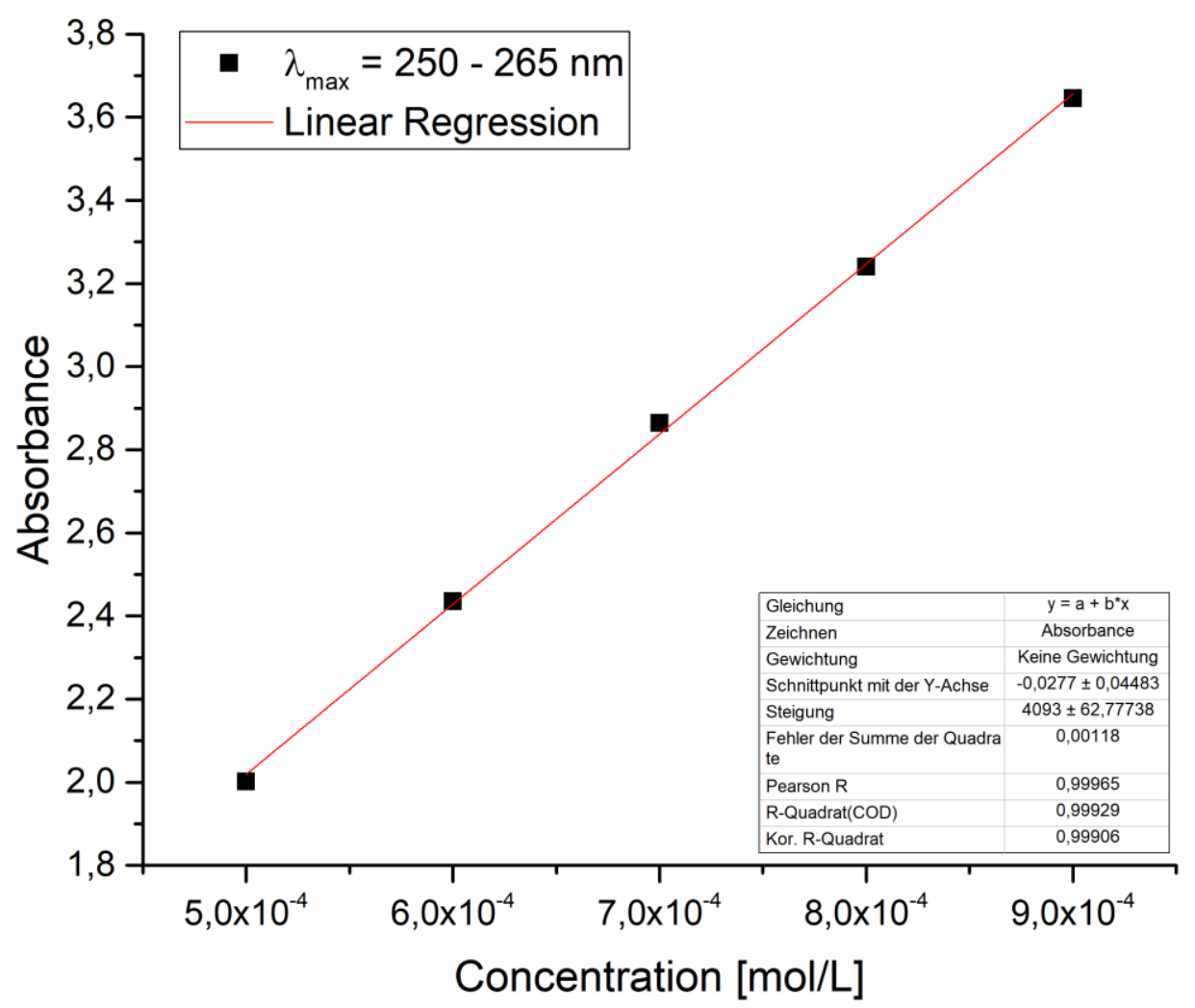

Supplementary Figure 25: Determination of extinction coefficient $\varepsilon\left(40930 \mathrm{M}^{-1} \mathrm{~cm}^{-1}\right)$ by linear regression of absorbance $\left(\lambda_{\max }=250-265 \mathrm{~nm}\right)$ of 9 against concentration. 


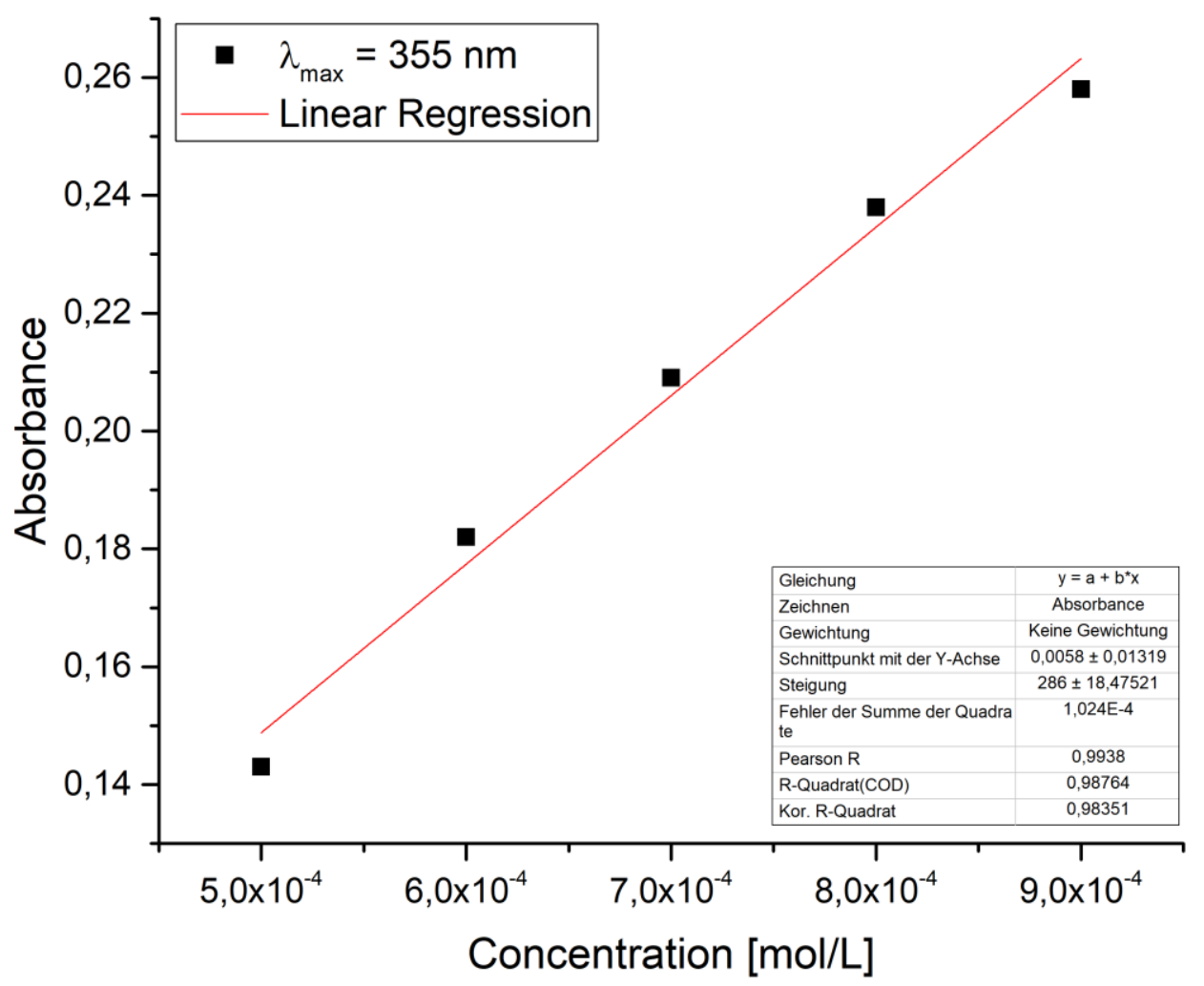

Supplementary Figure 26: Determination of extinction coefficient $\varepsilon\left(2860 \mathrm{M}^{-1} \mathrm{~cm}^{-1}\right)$ by linear regression of absorbance $\left(\lambda_{\max }=355 \mathrm{~nm}\right)$ of 9 against concentration. 


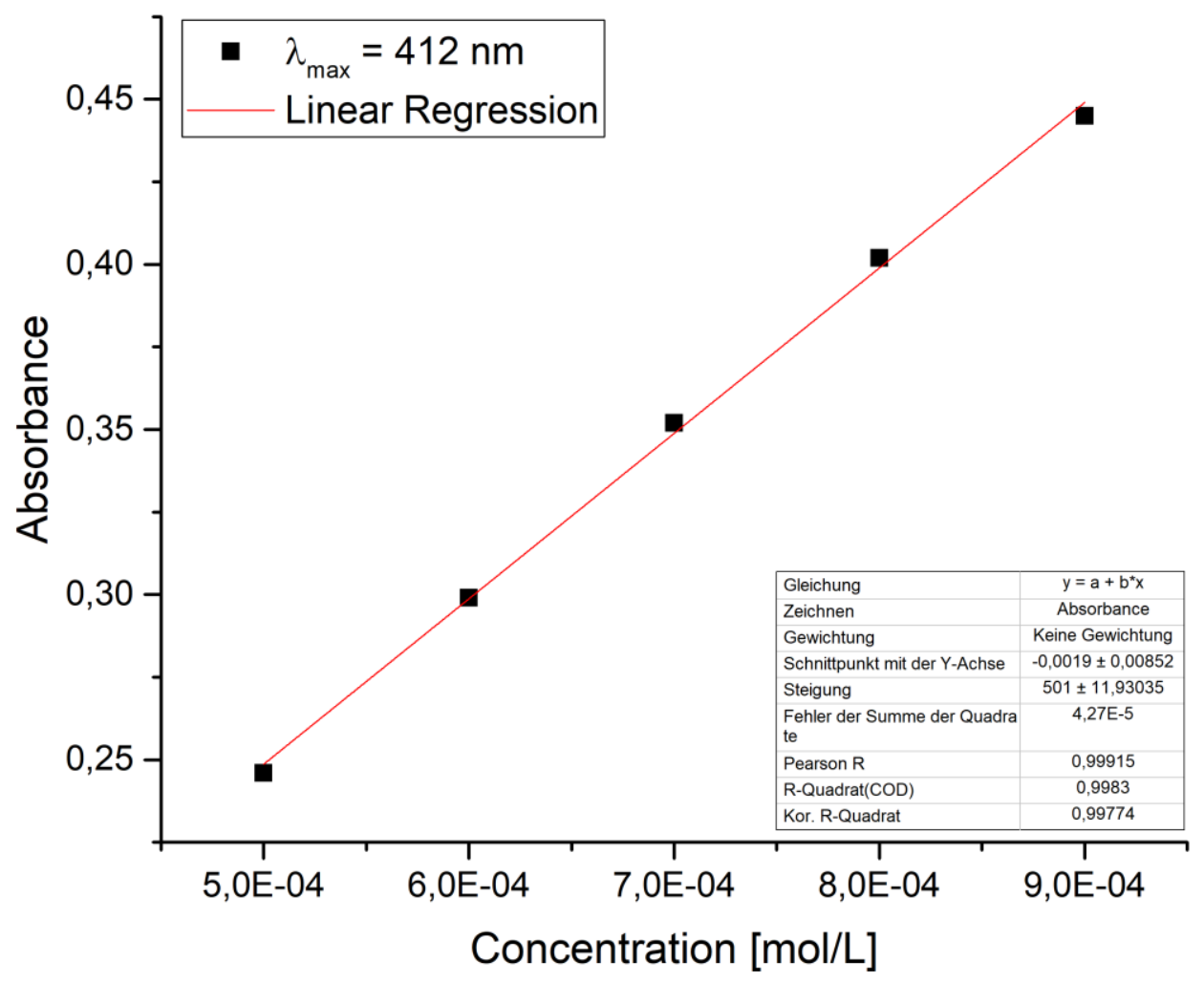

Supplementary Figure 27: Determination of extinction coefficient $\varepsilon\left(5010 \mathrm{M}^{-1} \mathrm{~cm}^{-1}\right)$ by linear regression of absorbance $\left(\lambda_{\max }=412 \mathrm{~nm}\right)$ of 9 against concentration. 


\section{Details on X-ray Diffraction Studies}

Supplementary Table 1: Crystal data and structure refinement for $[4]_{2} \cdot(\mathrm{THF})_{3}$ (CCDC-1953248).

Identification code

Empirical formula

Formula weight

Temperature

Wavelength

Crystal system

Space group

Unit cell dimensions

Volume

Z

Density (calculated)

Absorption coefficient

$\mathrm{F}(000)$

Crystal size

Theta range for data collection

Index ranges

Reflections collected

Independent reflections

Completeness to theta $=25.242^{\circ}$

Absorption correction

Max. and min. transmission

Refinement method

Data / restraints / parameters

Goodness-of-fit on $\mathrm{F}^{2}$

Final $R$ indices [l>2sigma(I)]

$R$ indices (all data)

Extinction coefficient

Largest diff. peak and hole sh3898

C132 H208 Li4 O3 Si10, 1.125 (C6 H14)

2248.57

152(2) K

$0.71073 \AA$

Triclinic

$\mathrm{P}-1$

$a=16.0030(11) \AA \quad \alpha=107.539(3)^{\circ}$.

$\mathrm{b}=29.9444(19) \AA \quad \beta=97.162(3)^{\circ}$.

$\mathrm{C}=31.757(2) \AA \quad \gamma=93.935(3)^{\circ}$.

14306.2(17) $\AA^{3}$

4

$1.044 \mathrm{Mg} / \mathrm{m}^{3}$

$0.138 \mathrm{~mm}^{-1}$

4929

$0.345 \times 0.276 \times 0.200 \mathrm{~mm}^{3}$

1.133 to $28.002^{\circ}$.

$-21<=\mathrm{h}<=21,-37<=\mathrm{k}<=39,-41<=\mathrm{k}<=41$

256396

$67386[R$ (int) $=0.0579]$

$99.4 \%$

Semi-empirical from equivalents

0.7456 and 0.6979

Full-matrix least-squares on $\mathrm{F}^{2}$

67386 / 282 / 2908

1.019

$\mathrm{R} 1=0.0716, \mathrm{wR} 2=0.1646$

$\mathrm{R} 1=0.1407, w R 2=0.1988$

$\mathrm{n} / \mathrm{a}$

0.935 and -0.619 e. $\AA^{-3}$ 


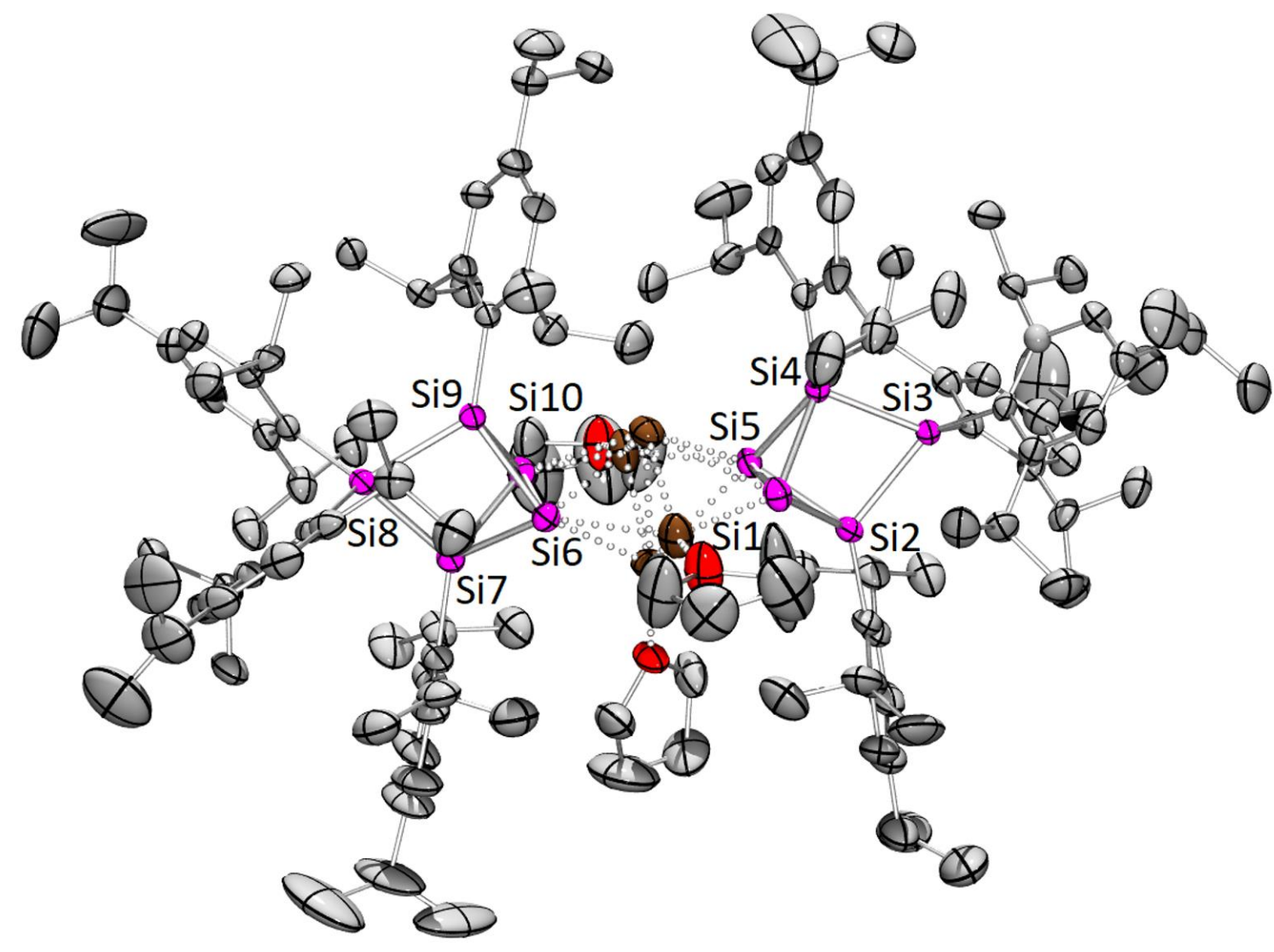

Supplementary Figure 28: Molecular structure of $[4]_{2} \cdot(\mathrm{THF})_{3}$ in the solid state Thermal ellipsoids are displayed at 50\% probability. Hydrogen atoms, and cocrystallized solvent molecules are omitted for clarity. Lithium bonds are drawn with dotted lines. Selected bond lengths [Å]: Si1-Si5 2.572(1), Si6-Si10 2.575(1), Si1-Si4 2.347(1), Si1-Li1 2.633(6), Si1-Li2 3.021(6), Li1-Li4 3.067(8), Li2-Li4 2.571(7). 
Supplementary Table 2: Crystal data and structure refinement for $[4]_{2} \cdot(\mathrm{THF})_{2}$ (CCDC-1953249).

Identification code

Empirical formula

Formula weight

Temperature

Wavelength

Crystal system

Space group

Unit cell dimensions

Volume

Z

Density (calculated)

Absorption coefficient

$\mathrm{F}(000)$

Crystal size

Theta range for data collection

Index ranges

Reflections collected

Independent reflections

Completeness to theta $=67.679^{\circ}$

Absorption correction

Max. and min. transmission

Refinement method

Data / restraints / parameters

Goodness-of-fit on $\mathrm{F}^{2}$

Final $R$ indices [l>2sigma(I)]

$R$ indices (all data)

Extinction coefficient

Largest diff. peak and hole sh4162cu

C128 H200 Li4 O2 Si10, 3(C6 H14)

2338.04

133(2) K

\section{$1.54178 \AA$}

Triclinic

P-1

$a=13.6454(5) \AA \quad \alpha=76.3140(10)^{\circ}$.

$\mathrm{b}=15.2125(5) \AA \quad \beta=74.2330(10)^{\circ}$.

$\mathrm{C}=19.0933(7) \AA \quad \gamma=76.5130(10)^{\circ}$.

$3645.0(2) \AA^{3}$

1

$1.065 \mathrm{Mg} / \mathrm{m}^{3}$

$1.197 \mathrm{~mm}^{-1}$

1286

$0.251 \times 0.083 \times 0.040 \mathrm{~mm}^{3}$

4.074 to $80.513^{\circ}$.

$-17<=\mathrm{h}<=17,-18<=\mathrm{k}<=19,-24<=\mathrm{k}<=24$

73063

$15601[\mathrm{R}(\mathrm{int})=0.0381]$

$99.4 \%$

Semi-empirical from equivalents

0.7543 and 0.6614

Full-matrix least-squares on $\mathrm{F}^{2}$

$15601 / 231 / 804$

1.049

$\mathrm{R} 1=0.0544, \mathrm{wR} 2=0.1504$

$R 1=0.0582, w R 2=0.1548$

$\mathrm{n} / \mathrm{a}$

0.961 and -0.758 e. $\AA^{-3}$ 


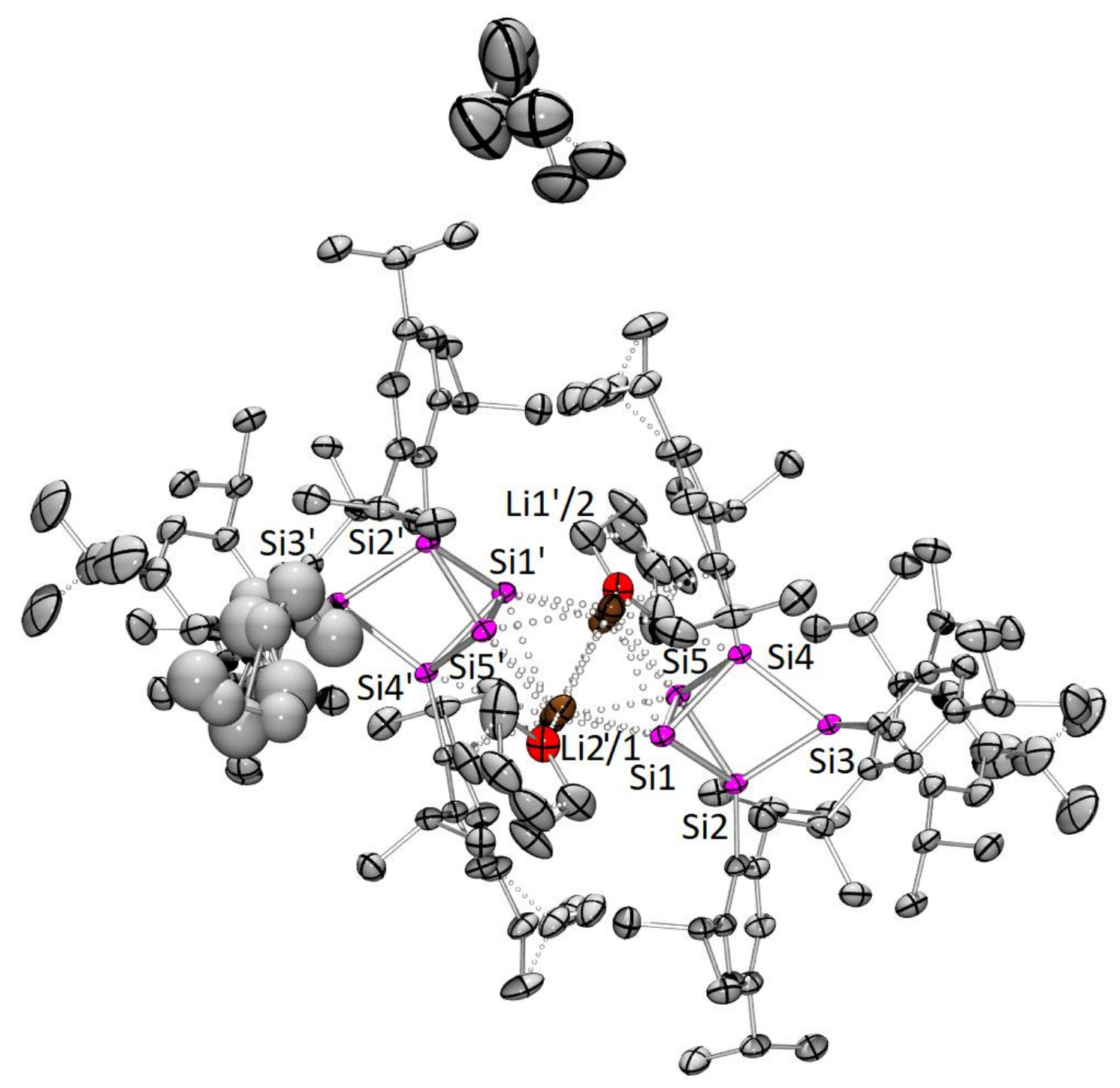

Supplementary Figure 29: Molecular structure of $[4]_{2} \cdot(\mathrm{THF})_{2}$ in the solid state Thermal ellipsoids are displayed at 50\% probability. Hydrogen atoms are omitted for clarity. Split positions are drawn with small dotted lines. Lithium bonds are drawn with bigger dotted lines. Selected bond lengths $[\AA]$ : Si1-Si5 2.5524(6), Si1-Si2 2.3822(6), Si1-Si4 2.3229(6), Si1-Li1 2.858(3), Si2-Li1 2.785(3), Li1-Li1'2.793(7). 
Supplementary Table 3: Crystal data and structure refinement for 7 (CCDC-1953250).

Identification code

Empirical formula

Formula weight

Temperature

Wavelength

Crystal system

Space group

Unit cell dimensions

Volume

Z

Density (calculated)

Absorption coefficient

$\mathrm{F}(000)$

Crystal size

Theta range for data collection

Index ranges

Reflections collected

Independent reflections

Completeness to theta $=25.242^{\circ}$

Absorption correction

Max. and min. transmission

Refinement method

Data / restraints / parameters

Goodness-of-fit on $\mathrm{F}^{2}$

Final $R$ indices [l>2sigma(I)]

$\mathrm{R}$ indices (all data)

Extinction coefficient

Largest diff. peak and hole sh4075

C66 H110 Si7, C5 H12

1172.31

142(2) K

$0.71073 \AA$

Monoclinic

$\mathrm{P} 21 / \mathrm{c}$

$a=24.7021(9) \AA \quad \alpha=90^{\circ}$.

$\mathrm{b}=27.0801(8) \AA \quad \beta=113.3692(15)^{\circ}$.

$\mathrm{c}=24.4934(7) \AA \quad \gamma=90^{\circ}$.

15040.4(8) $\AA^{3}$

8

$1.035 \mathrm{Mg} / \mathrm{m}^{3}$

$0.163 \mathrm{~mm}^{-1}$

5168

$0.522 \times 0.494 \times 0.376 \mathrm{~mm}^{3}$

1.171 to $29.689^{\circ}$.

$-31<=\mathrm{h}<=34,-35<=\mathrm{k}<=37,-34<=\mid<=33$

133330

$42515[R($ int $)=0.0677]$

$100.0 \%$

Semi-empirical from equivalents

0.7459 and 0.6764

Full-matrix least-squares on $\mathrm{F}^{2}$

42515 / 290 / 1522

1.024

$\mathrm{R} 1=0.0739, \mathrm{wR} 2=0.1395$

$\mathrm{R} 1=0.1520, \mathrm{wR} 2=0.1733$

$\mathrm{n} / \mathrm{a}$

0.917 and -0.823 e. $\AA^{-3}$ 


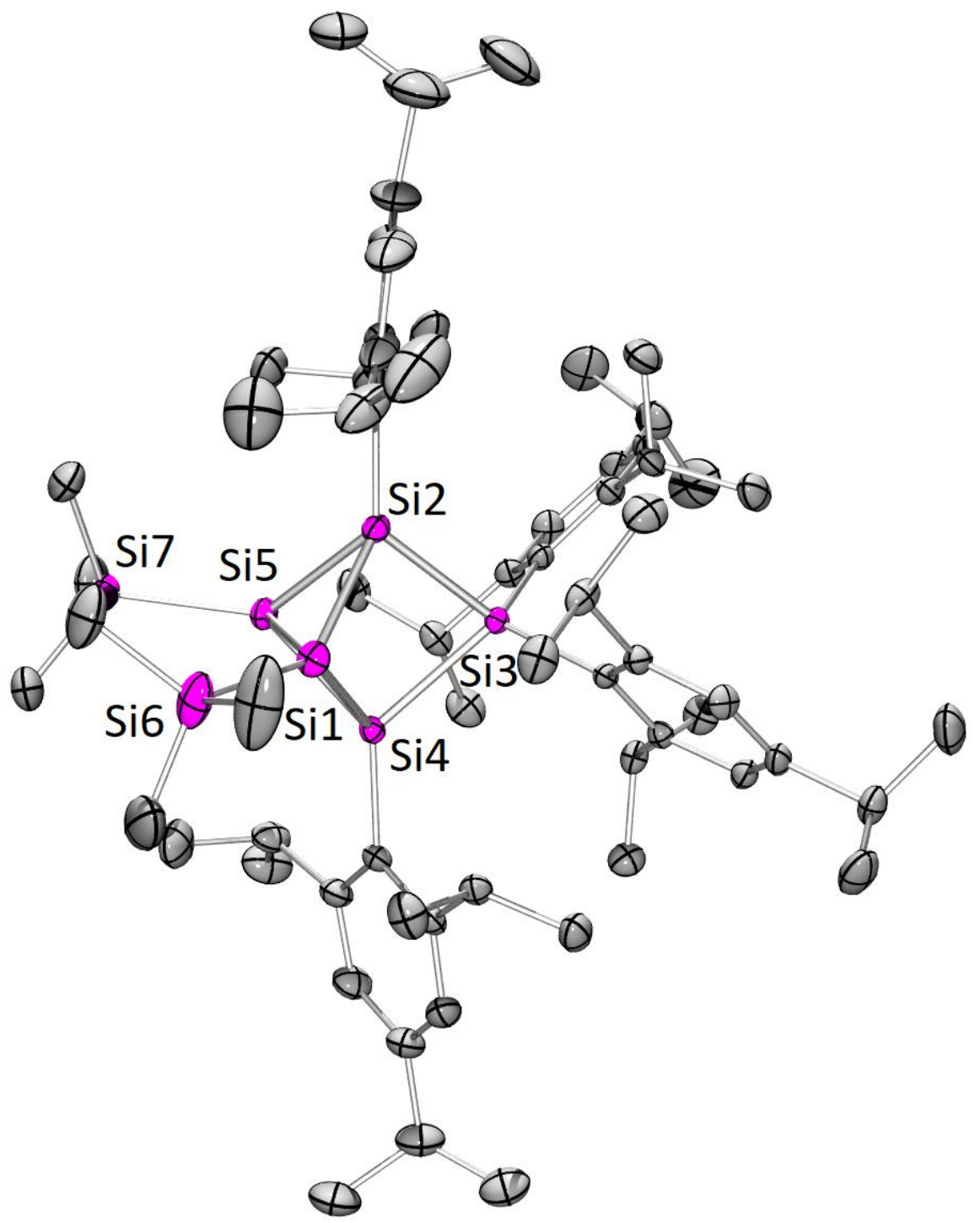

Supplementary Figure 30: Molecular structure of $\mathbf{7}$ in the solid state. Thermal ellipsoids are displayed at 50\% probability. Hydrogen atoms and co-crystallized solvent molecules are omitted for clarity. Selected bond lengths [Å]: Si1-Si5 2.469(1), Si1-Si2 2.302(1) Si1-Si4 2.338(1), Si1-Si6 2.332(1), Si2-Si3 2.376(1). 
Supplementary Table 4: Crystal data and structure refinement for 8 (CCDC-1953251).

Identification code

Empirical formula

Formula weight

Temperature

Wavelength

Crystal system

Space group

Unit cell dimensions

Volume

Z

Density (calculated)

Absorption coefficient

$\mathrm{F}(000)$

Crystal size

Theta range for data collection

Index ranges

Reflections collected

Independent reflections

Completeness to theta $=25.242^{\circ}$

Absorption correction

Max. and min. transmission

Refinement method

Data / restraints / parameters

Goodness-of-fit on $\mathrm{F}^{2}$

Final $R$ indices [l>2sigma(I)]

$\mathrm{R}$ indices (all data)

Extinction coefficient

Largest diff. peak and hole sh4060

C66 H106 B N Si5

1064.77

132(2) K

$0.71073 \AA$

Triclinic

P-1

$a=13.5006(6) \AA \quad \alpha=98.387(2)^{\circ}$.

$b=13.5798(6) \AA \quad \beta=92.938(2)^{\circ}$.

$\mathrm{C}=18.9871(8) \AA \quad \gamma=106.305(2)^{\circ}$.

3289.7(3) $\AA^{3}$

2

$1.075 \mathrm{Mg} / \mathrm{m}^{3}$

$0.146 \mathrm{~mm}^{-1}$

1168

$0.652 \times 0.377 \times 0.120 \mathrm{~mm}^{3}$

1.089 to $30.988^{\circ}$.

$-19<=\mathrm{h}<=19,-19<=\mathrm{k}<=19,-27<=\mathrm{k}<=27$

76421

$20730[R($ int $)=0.0277]$

$100.0 \%$

Semi-empirical from equivalents

0.7461 and 0.7190

Full-matrix least-squares on $\mathrm{F}^{2}$

20730 / 0 / 1082

1.027

$\mathrm{R} 1=0.0373, \mathrm{wR} 2=0.0888$

$R 1=0.0527, w R 2=0.0965$

$\mathrm{n} / \mathrm{a}$

0.402 and -0.228 e. $\AA^{-3}$ 


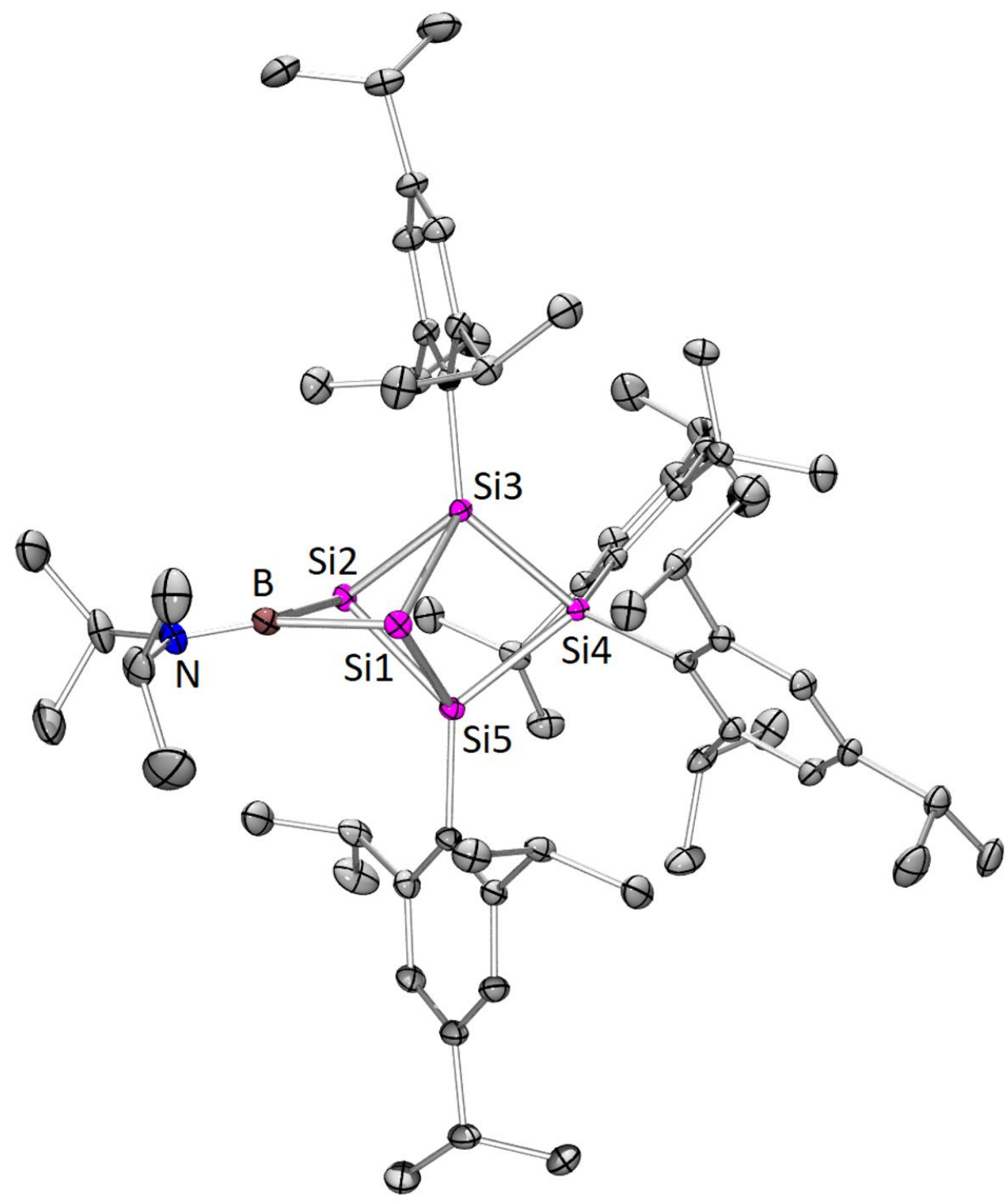

Supplementary Figure 31: Molecular structure of 8 in the solid state. Thermal ellipsoids are displayed at $50 \%$ probability. Hydrogen atoms are omitted for clarity. Selected bond lengths [Å]: Si1-B 2.031(1), Si1-Si2 2.5911(4), Si1-Si3 2.3270(4), Si3Si4 2.3816(4), B-N 1.374(2). 
Supplementary Table 5: Crystal data and structure refinement for 9 (CCDC-1953252).

Identification code

Empirical formula

Formula weight

Temperature

Wavelength

Crystal system

Space group

Unit cell dimensions

Volume

Z

Density (calculated)

Absorption coefficient

$\mathrm{F}(000)$

Crystal size

Theta range for data collection

Index ranges

Reflections collected

Independent reflections

Completeness to theta $=25.242^{\circ}$

Absorption correction

Max. and min. transmission

Refinement method

Data / restraints / parameters

Goodness-of-fit on $\mathrm{F}^{2}$

Final $\mathrm{R}$ indices [l>2sigma(I)]

$\mathrm{R}$ indices (all data)

Extinction coefficient

Largest diff. peak and hole sh4073

C66 H106 N P Si5

1084.93

142(2) $\mathrm{K}$

$0.71073 \AA$

Monoclinic

$\mathrm{P} 21 / \mathrm{n}$

$a=20.5557(11) \AA \quad \alpha=90^{\circ}$.

$b=13.3994(10) \AA \quad \beta=90.261(2)^{\circ}$.

$\mathrm{c}=24.2831(17) \AA \quad \gamma=90^{\circ}$.

6688.3(8) $\AA^{3}$

4

$1.077 \mathrm{Mg} / \mathrm{m}^{3}$

$0.168 \mathrm{~mm}^{-1}$

2376

$0.201 \times 0.200 \times 0.184 \mathrm{~mm}^{3}$

1.301 to $26.615^{\circ}$.

$-20<=\mathrm{h}<=25,-16<=\mathrm{k}<=16,-30<=\mathrm{k}<=29$

101267

$13657[R($ int $)=0.0857]$

$99.6 \%$

Semi-empirical from equivalents

0.7454 and 0.7211

Full-matrix least-squares on $\mathrm{F}^{2}$

13657 / 601 / 899

1.383

$\mathrm{R} 1=0.0776, \mathrm{wR} 2=0.1784$

$R 1=0.1617, w R 2=0.2022$

$\mathrm{n} / \mathrm{a}$

0.544 and -0.456 e. $\AA^{-3}$ 


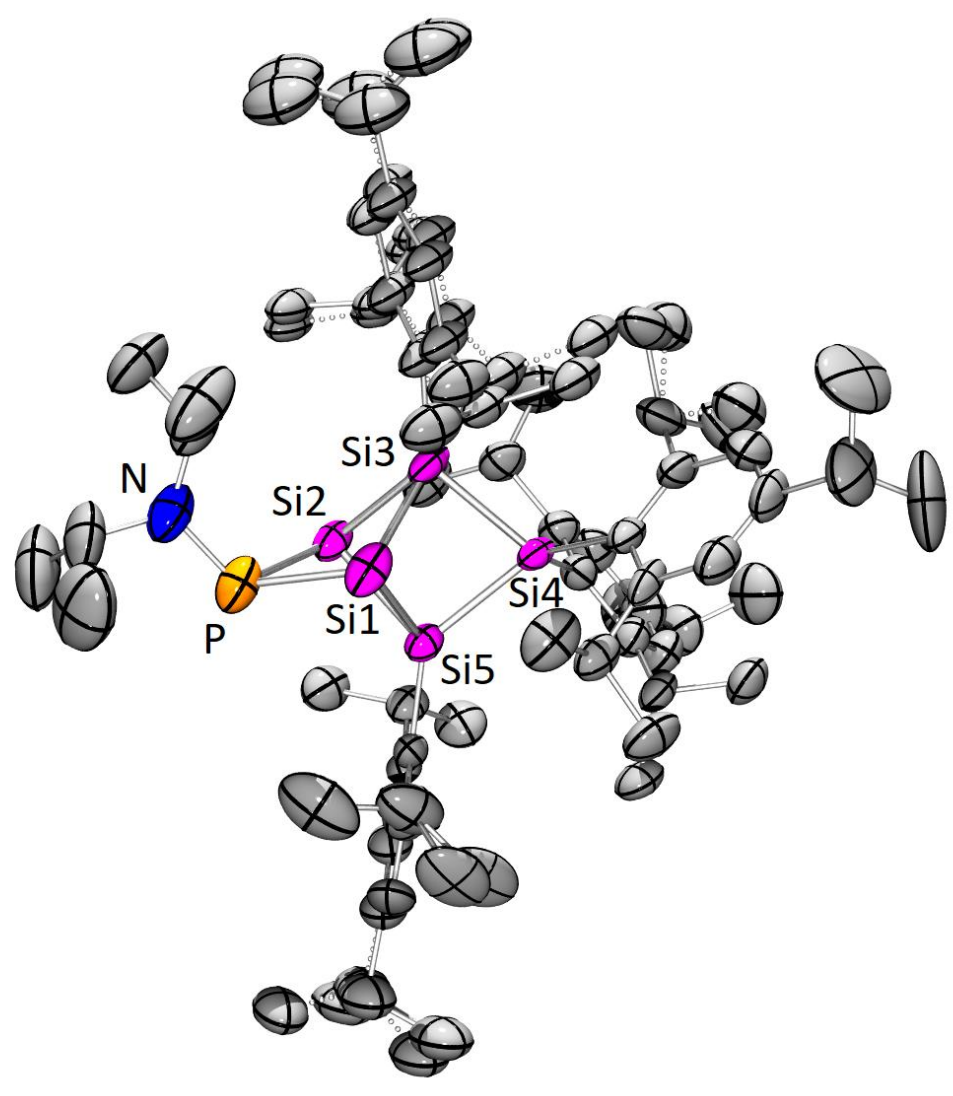

Supplementary Figure 32: Molecular structure of 9 in the solid state. Thermal ellipsoids are displayed at $50 \%$ probability. Hydrogen atoms and co-crystallized solvent molecules are omitted for clarity. Split positions are drawn with dotted lines. Selected bond lengths [Å]: Si1-P 2.314(2), Si1-Si2 2.613(1), Si1-Si3 2.391(2), Si3-Si4 2.38(1), P-N 1.651(3).

\section{Comment on structure refinement:}

Level B alerts caused by many positionally disordered parts of the molecule, which were refined on split positions. All attempts treating the problem as pseudomerohedral twinning in the higher orthorhombic symmetry remained unsuccessful. 


\section{Computational Details}

Structural optimizations were carried out using the Gaussian 09 program package ${ }^{[2]}$ and the functional BP86 ${ }^{[3,4]}$ with Grimme dispersion corrections D3 ${ }^{[5]}$ in combination with the def2-SVP basis set. ${ }^{[6]}$ To ensure the presence of a local minimum a subsequent frequency analysis at the same level was performed for each structure. Optimized structures were plotted using ChemCraft 1.8. ${ }^{[7]}$ Molecular orbitals were calculated using the functional M06-2X ${ }^{[8]}$ with the Grimme dispersion corrections D3 ${ }^{[5]}$ in combination with the def2-SVPP basis set. ${ }^{[6]}$ Chemical shifts were calculated with $\mathrm{GIAO}^{[9-13]}$ as implemented in the Gaussian 09 program package using the functional OLYP ${ }^{[14-17]}$ in combination with the two different basis sets def2-TZVPP ${ }^{[18]}$ and pcS$2 .{ }^{[19-21]}$ All chemical shifts were referenced to the values calculated for $\mathrm{Si}\left(\mathrm{CH}_{3}\right)_{4}$, $\mathrm{H}_{3} \mathrm{PO}_{4}$ and $\mathrm{BF}_{3} \cdot \mathrm{Et}_{2} \mathrm{O}$ at the same level of theory.

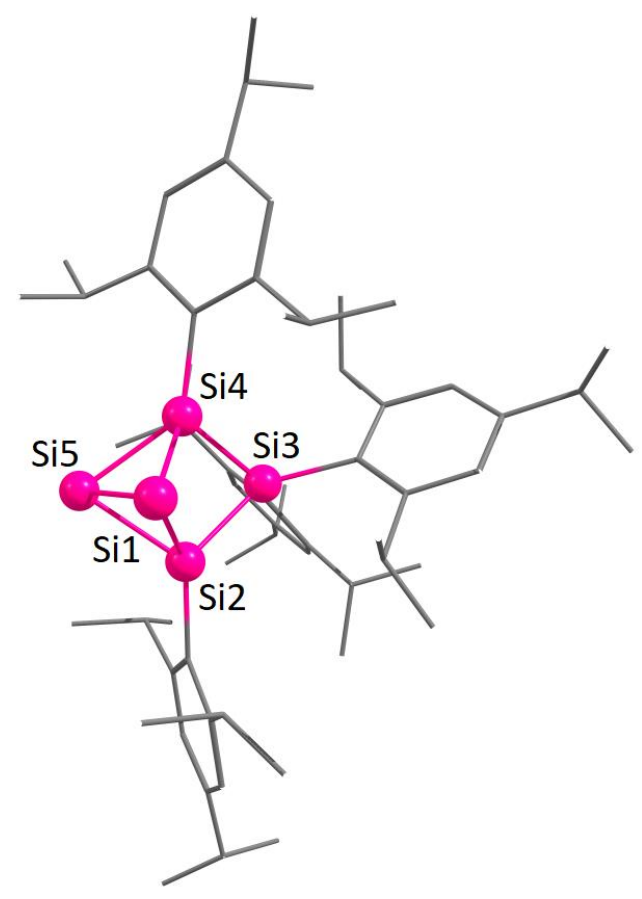

Supplementary Figure 33: Optimized structure of 4' (4 was calculated as the monomeric dianionic Sis-cluster core without lithium counter cations and coordinated donor molecules; level of theory: BP86/def2-SVP) 


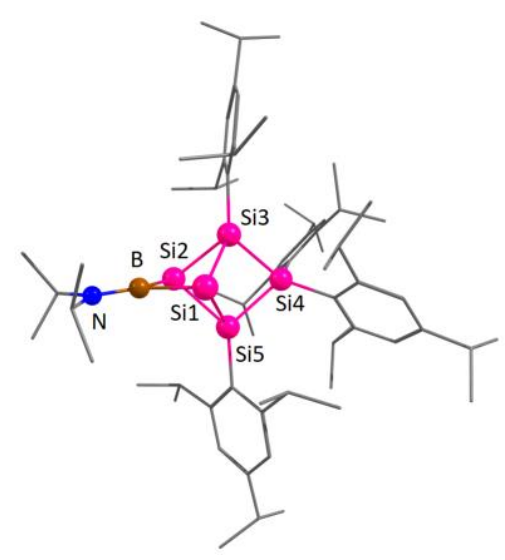

8

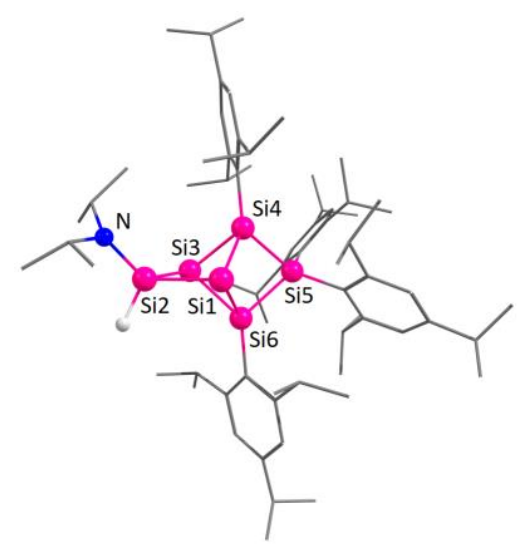

10

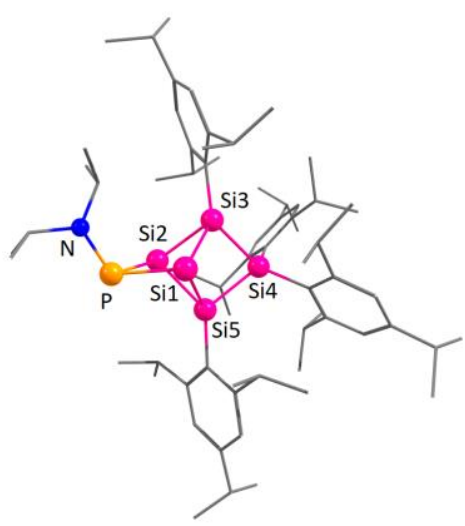

9

Supplementary Figure 34: Optimized structures of 8, 10 and 9 (level of theory: BP86/def2-SVP).

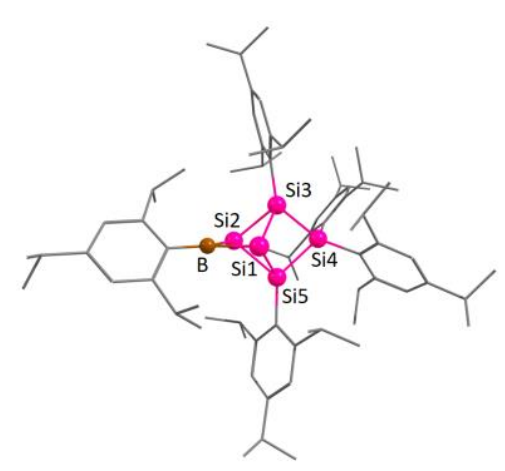

${ }^{8}$ Tip

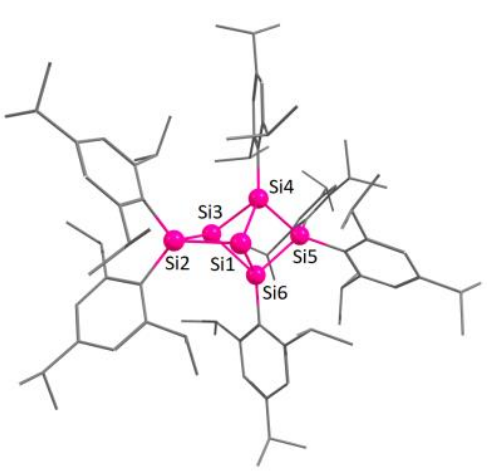

2

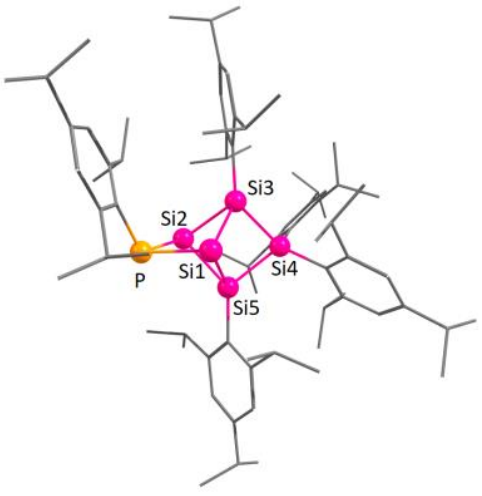

$9_{\text {Tip }}$

Supplementary Figure 35: Optimized structures of 8 Tip, 2 and 9 Tip (level of theory: BP86/def2-SVP). 
Supplementary Table 6: Atomic coordinates of the optimized structure of 4'.

$\begin{array}{lrrr}\text { C } & -3.697038000 & -2.772747000 & -2.417484000 \\ \text { C } & 3.665859000 & -4.319457000 & -2.403690000 \\ \text { C } & -0.242282000 & -4.138826000 & -0.884223000 \\ \text { C } & -3.707128000 & -4.973775000 & 3.290269000 \\ \text { C } & 3.106647000 & -2.668191000 & -4.240802000 \\ \text { C } & -4.007807000 & -2.734506000 & 0.099492000 \\ \text { C } & 3.147241000 & -2.907602000 & -2.716404000 \\ \text { C } & 1.594819000 & -4.144551000 & 0.877587000 \\ \text { C } & -3.481618000 & -1.965218000 & -1.122137000 \\ \text { C } & -1.705298000 & -4.896834000 & 4.861343000 \\ \text { C } & -0.494820000 & -3.315623000 & 0.188480000 \\ \text { C } & -2.709498000 & -4.064530000 & 4.039060000 \\ \text { C } & -1.112097000 & -3.565163000 & 2.104446000 \\ \text { C } & -8.477308000 & 0.167122000 & -1.464514000 \\ \text { C } & -5.425136000 & -0.357879000 & -1.010282000 \\ \text { C } & -2.019595000 & -3.105342000 & 3.074663000 \\ \text { C } & -0.488585000 & -2.697945000 & 1.184437000 \\ \text { C } & -4.059223000 & -0.560041000 & -1.292620000 \\ \text { C } & 3.907913000 & -1.772198000 & -2.036389000 \\ \text { C } & 5.272916000 & -1.937692000 & -1.719201000 \\ \text { C } & 7.635139000 & -1.028160000 & 0.775811000 \\ \text { C } & -7.493380000 & 1.122823000 & -0.759673000 \\ \text { C } & -6.046728000 & 0.892778000 & -1.185047000 \\ \text { C } & -3.255575000 & 0.522199000 & -1.781095000 \\ \text { C } & -2.320089000 & -1.733226000 & 3.078265000 \\ \text { C } & -0.782080000 & -1.298624000 & 1.201312000 \\ \text { C } & 7.493603000 & -1.123112000 & -0.758877000 \\ \text { C } & 3.255777000 & -0.522847000 & -1.780509000 \\ \text { C } & -7.633855000 & 1.030796000 & 0.775302000 \\ \text { C } & 6.046831000 & -0.893353000 & -1.183995000 \\ \text { C } & -1.744583000 & -0.835731000 & 2.154883000 \\ \text { C } & -5.272792000 & 1.936968000 & -1.720500000\end{array}$




$\begin{array}{lrrr}\text { C } & -3.907742000 & 1.771442000 & -2.037465000 \\ \text { C } & -3.106317000 & 2.666739000 & -4.242093000 \\ \text { C } & 1.663214000 & -1.296054000 & 3.528583000 \\ \text { C } & 5.425285000 & 0.357399000 & -1.009752000 \\ \text { C } & 4.059409000 & 0.559518000 & -1.292276000 \\ \text { C } & 8.477343000 & -0.169072000 & -1.466260000 \\ \text { C } & -3.705440000 & 0.808103000 & 2.079636000 \\ \text { C } & 2.182661000 & -0.630123000 & 2.238686000 \\ \text { C } & 3.705160000 & -0.807437000 & 2.080562000 \\ \text { C } & -3.147089000 & 2.906676000 & -2.717780000 \\ \text { C } & -2.182970000 & 0.630829000 & 2.238096000 \\ \text { C } & 0.781872000 & 1.298955000 & 1.201147000 \\ \text { C } & 3.481804000 & 1.964750000 & -1.122286000 \\ \text { C } & 1.744292000 & 0.836401000 & 2.154959000 \\ \text { C } & -3.665842000 & 4.318599000 & -2.405605000 \\ \text { C } & -1.663786000 & 1.297189000 & 3.527876000 \\ \text { C } & 3.697381000 & 2.771861000 & -2.417871000 \\ \text { C } & 0.488362000 & 2.698270000 & 1.183774000 \\ \text { C } & -0.494867000 & 3.315610000 & 0.187447000 \\ \text { C } & 4.007851000 & 2.734433000 & 0.099149000 \\ \text { C } & 2.319711000 & 1.734218000 & 3.078085000 \\ \text { C } & 0.242486000 & 4.138430000 & -0.885373000 \\ \text { C } & -1.595000000 & 4.144799000 & 0.876012000 \\ \text { C } & 1.111700000 & 3.565802000 & 2.103601000 \\ \text { C } & 2.019125000 & 3.106320000 & 3.074038000 \\ \text { C } & 2.708931000 & 4.065847000 & 4.038159000 \\ \text { C } & 1.704671000 & 4.898324000 & 4.860198000 \\ \text { C } & 3.706465000 & 4.975017000 & 3.289103000 \\ \text { H } & -3.260199000 & -3.790858000 & -2.322212000 \\ \text { H } & 2.943436000 & -5.080557000 & -2.769227000 \\ \text { H } & 2.497001000 & -3.447758000 & -4.746886000 \\ \text { H } & -3.172349000 & -5.622532000 & 2.563707000 \\ \text { H } & -4.783726000 & -2.875777000 & -2.640212000 \\ \text { H } & -0.842010000 & -4.959538000 & -0.428247000\end{array}$




$\begin{array}{llll}\mathrm{H} & -1.481776000 & -4.589594000 & -1.596859000 \\ \mathrm{H} & -4.267457000 & -5.633520000 & 3.990549000 \\ \mathrm{H} & 4.641201000 & -4.531571000 & -2.898185000 \\ \mathrm{H} & -3.192645000 & -2.273161000 & -3.270549000 \\ \mathrm{H} & 4.135663000 & -2.682138000 & -4.667373000 \\ \mathrm{H} & -4.435003000 & -4.368284000 & 2.712485000 \\ \mathrm{H} & -0.907852000 & -3.482885000 & -1.482320000 \\ \mathrm{H} & -3.387930000 & -3.640041000 & 0.267170000 \\ \mathrm{H} & -5.062429000 & -3.070534000 & -0.030221000 \\ \mathrm{H} & 3.802795000 & -4.476070000 & -1.313119000 \\ \mathrm{H} & -1.092063000 & -5.546466000 & 4.201019000 \\ \mathrm{H} & 1.186244000 & -5.043039000 & 1.391993000 \\ \mathrm{H} & 2.331694000 & -4.499818000 & 0.128390000 \\ \mathrm{H} & -0.876119000 & -4.642646000 & 2.057698000 \\ \mathrm{H} & 2.639290000 & -1.688067000 & -4.469425000 \\ \mathrm{H} & -2.226664000 & -5.556776000 & 5.590296000 \\ \mathrm{H} & -8.266163000 & -0.890054000 & -1.195652000 \\ \mathrm{H} & 2.089195000 & -2.828107000 & -2.369346000 \\ \mathrm{H} & -6.015393000 & -1.202455000 & -0.616161000 \\ \mathrm{H} & -8.392250000 & 0.251322000 & -2.567929000 \\ \mathrm{H} & -2.383031000 & -1.839370000 & -0.999061000 \\ \mathrm{H} & 5.758274000 & -2.914568000 & -1.887703000 \\ \mathrm{H} & -3.947289000 & -2.124497000 & 1.022390000 \\ \mathrm{H} & -9.530555000 & 0.382426000 & -1.175503000 \\ \mathrm{H} & 6.938840000 & -1.729457000 & 1.280064000 \\ \mathrm{H} & 0.988955000 & -2.476428000 & -0.353499000 \\ \mathrm{H} & 2.142009000 & -3.544485000 & 1.632881000 \\ \mathrm{H} & -3.294853000 & -3.441814000 & 4.754789000 \\ \mathrm{H} & -1.006351000 & -4.239525000 & 5.418984000 \\ \mathrm{H} & 8.672974000 & -1.256570000 & 1.108747000 \\ \mathrm{H} & 7.762643000 & -2.163186000 & -1.057508000 \\ \mathrm{H} & -7.376603000 & 0.009856000 & 1.130257000 \\ & 7.379235000 & -0.006254000 & 1.128981000 \\ -2.638794000 & 1.686603000 & -4.470320000\end{array}$




$\begin{array}{lrrr}\mathrm{H} & -7.762932000 & 2.162238000 & -1.060138000 \\ \mathrm{H} & -8.671701000 & 1.258816000 & 1.108476000 \\ \mathrm{H} & -3.047487000 & -1.354553000 & 3.818836000 \\ \mathrm{H} & 1.917340000 & -2.377774000 & 3.547778000 \\ \mathrm{H} & -4.135299000 & 2.680377000 & -4.668757000 \\ \mathrm{H} & 3.970415000 & -1.887074000 & 2.084932000 \\ \mathrm{H} & 1.723376000 & -1.158226000 & 1.379664000 \\ \mathrm{H} & -4.059150000 & 0.383565000 & 1.119811000 \\ \mathrm{H} & -6.937938000 & 1.733800000 & 1.277703000 \\ \mathrm{H} & -5.758170000 & 2.913776000 & -1.889333000 \\ \mathrm{H} & -4.271503000 & 0.322388000 & 2.905116000 \\ \mathrm{H} & 0.560604000 & -1.211235000 & 3.603959000 \\ \mathrm{H} & 9.530710000 & -0.383739000 & -1.177203000 \\ \mathrm{H} & -2.496740000 & 3.446215000 & -4.748401000 \\ \mathrm{H} & 4.059042000 & -0.383088000 & 1.120716000 \\ \mathrm{H} & 8.391830000 & -0.255791000 & -2.569446000 \\ \mathrm{H} & -2.089074000 & 2.827402000 & -2.370575000 \\ \mathrm{H} & 8.266259000 & 0.888706000 & -1.199732000 \\ \mathrm{H} & 2.113657000 & -0.823384000 & 4.429833000 \\ \mathrm{H} & -1.723536000 & 1.158664000 & 1.378993000 \\ \mathrm{H} & 6.015567000 & 1.202087000 & -0.615908000 \\ \mathrm{H} & -3.970693000 & 1.887740000 & 2.083746000 \\ \mathrm{H} & 4.271052000 & -0.321544000 & 2.906054000 \\ \mathrm{H} & 3.193094000 & 2.271999000 & -3.270835000 \\ \mathrm{H} & 2.383205000 & 1.838933000 & -0.999284000 \\ \mathrm{H} & -4.641102000 & 4.530513000 & -2.900348000 \\ \mathrm{H} & -2.114369000 & 0.824780000 & 4.429191000 \\ \mathrm{H} & -0.988894000 & 2.476226000 & -0.354335000 \\ \mathrm{H} & -0.561183000 & 1.212477000 & 3.603486000 \\ \mathrm{H} & 4.784098000 & 2.874815000 & -2.640483000 \\ \mathrm{H} & -3.802993000 & 4.475542000 & -1.315108000 \\ \mathrm{H} & -2.943388000 & 5.079619000 & -2.771244000 \\ \mathrm{H} & 3.947350000 & 2.124701000 & 1.022235000 \\ \mathrm{H} & -1.917994000 & 2.378898000 & 3.546677000\end{array}$




$\begin{array}{lrrr}\mathrm{H} & 0.908501000 & 3.482340000 & -1.482799000 \\ \mathrm{H} & 3.260524000 & 3.789999000 & -2.322986000 \\ \mathrm{H} & 3.047091000 & 1.355816000 & 3.818813000 \\ \mathrm{H} & 5.062444000 & 3.070533000 & -0.030600000 \\ \mathrm{H} & -2.142419000 & 3.545018000 & 1.631369000 \\ \mathrm{H} & -0.481371000 & 4.588621000 & -1.598577000 \\ \mathrm{H} & -2.331667000 & 4.499844000 & 0.126506000 \\ \mathrm{H} & 3.387866000 & 3.639955000 & 0.266496000 \\ \mathrm{H} & 0.841793000 & 4.959532000 & -0.429555000 \\ \mathrm{H} & -1.186521000 & 5.043445000 & 1.390217000 \\ \mathrm{H} & 1.005819000 & 4.241164000 & 5.418134000 \\ \mathrm{H} & 0.875688000 & 4.643253000 & 2.056512000 \\ \mathrm{H} & 3.294364000 & 3.443406000 & 4.754059000 \\ \mathrm{H} & 1.091324000 & 5.547641000 & 4.199660000 \\ \mathrm{H} & 2.225985000 & 5.558626000 & 5.588868000 \\ \mathrm{H} & 4.434246000 & 4.369472000 & 2.711258000 \\ \mathrm{H} & 3.171536000 & 5.623690000 & 2.562575000 \\ \mathrm{H} & 4.266915000 & 5.634829000 & 3.989214000 \\ \mathrm{Si} & -0.289113000 & -1.253126000 & -3.435604000 \\ \mathrm{Si} & 1.334544000 & -0.309578000 & -1.948715000 \\ \mathrm{Si} & -1.334280000 & 0.309041000 & -1.948905000 \\ \mathrm{Si} & 0.289503000 & 1.252165000 & -3.435943000 \\ \mathrm{Si} & 0.000040000 & -0.000002000 & -0.040809000\end{array}$


Supplementary Table 7: Atomic coordinates of the optimized structure of 8.

$\begin{array}{lrrr}\text { C } & 2.243354000 & -6.203454000 & -3.925362000 \\ \text { C } & 7.709839000 & -1.254569000 & 1.524389000 \\ \text { C } & -1.731665000 & -5.631490000 & 4.787893000 \\ \text { C } & 1.559395000 & -4.287978000 & 1.087387000 \\ \text { C } & 2.448660000 & -4.725891000 & -4.312830000 \\ \text { C } & 3.626302000 & -2.883013000 & 0.566796000 \\ \text { C } & 8.412978000 & 1.190616000 & 1.381760000 \\ \text { C } & 3.944931000 & -4.392857000 & -4.495205000 \\ \text { C } & 2.153313000 & -3.800187000 & -1.951644000 \\ \text { C } & 7.566320000 & 0.071372000 & 0.746043000 \\ \text { C } & 2.095890000 & -3.000727000 & 0.429553000 \\ \text { C } & 1.804114000 & -3.772769000 & -3.313111000 \\ \text { C } & 1.652543000 & -1.721600000 & 3.989229000 \\ \text { C } & -2.749182000 & -4.781220000 & 4.002757000 \\ \text { C } & 1.618932000 & -2.889189000 & -1.021169000 \\ \text { C } & 6.093886000 & 0.443109000 & 0.610315000 \\ \text { C } & -2.075236000 & -3.809233000 & 3.041258000 \\ \text { C } & -3.707946000 & -4.029756000 & 4.950725000 \\ \text { C } & -1.117064000 & -2.882931000 & 3.489223000 \\ \text { C } & 5.353476000 & 0.914357000 & 1.711610000 \\ \text { C } & 5.421365000 & 0.273656000 & -0.610771000 \\ \text { C } & 3.861101000 & 1.474473000 & 4.190455000 \\ \text { C } & -1.887078000 & -4.166349000 & -1.372370000 \\ \text { C } & 0.881735000 & -2.798999000 & -3.726369000 \\ \text { C } & -2.432251000 & -3.768883000 & 1.684254000 \\ \text { C } & 3.910262000 & -0.851837000 & -2.935371000 \\ \text { C } & 0.526988000 & -1.001851000 & 3.224657000 \\ \text { C } & -0.502800000 & -1.957688000 & 2.623835000 \\ \text { C } & 0.655645000 & -1.922178000 & -1.449305000 \\ \text { C } & 3.974740000 & 1.182316000 & 1.624877000 \\ \text { C } & -1.868067000 & -2.841480000 & 0.784967000 \\ \text { C } & 4.038490000 & 0.511293000 & -0.745485000\end{array}$




$\begin{array}{lrrr}\text { C } & -0.866289000 & -1.932426000 & 1.244644000 \\ \text { C } & 0.298487000 & -1.881909000 & -2.828078000 \\ \text { C } & 3.242236000 & 1.791097000 & 2.819883000 \\ \text { C } & 3.291909000 & 0.949288000 & 0.390594000 \\ \text { C } & -0.159041000 & 0.057618000 & 4.107457000 \\ \text { C } & -2.352761000 & -2.876738000 & -0.667069000 \\ \text { C } & 3.397626000 & 0.348832000 & -2.123244000 \\ \text { C } & -3.874490000 & -2.669832000 & -0.796656000 \\ \text { C } & 3.106083000 & 3.317455000 & 2.624764000 \\ \text { C } & 3.553605000 & 1.660114000 & -2.920062000 \\ \text { C } & -0.686936000 & -0.862756000 & -3.397076000 \\ \text { C } & -1.838058000 & -1.506038000 & -4.190975000 \\ \text { C } & 0.048024000 & 0.197872000 & -4.237688000 \\ \text { C } & -4.059940000 & -0.784778000 & 2.756096000 \\ \text { C } & -3.497489000 & 0.437407000 & 2.011507000 \\ \text { C } & -3.597437000 & 1.706838000 & 2.881460000 \\ \text { C } & -3.370325000 & 1.133919000 & -0.480390000 \\ \text { C } & -4.135182000 & 0.698549000 & 0.646668000 \\ \text { C } & 2.153942000 & 5.817374000 & -1.522096000 \\ \text { C } & -3.918296000 & 1.752246000 & -4.260449000 \\ \text { C } & -3.284861000 & 2.036394000 & -2.888668000 \\ \text { C } & -4.043779000 & 1.450375000 & -1.698973000 \\ \text { C } & 1.420039000 & 6.731028000 & 1.896611000 \\ \text { C } & -5.529766000 & 0.555477000 & 0.518854000 \\ \text { C } & 0.125843000 & 6.063658000 & 1.405386000 \\ \text { C } & 0.741458000 & 6.193705000 & -1.051081000 \\ \text { C } & -5.440577000 & 1.280900000 & -1.777385000 \\ \text { C } & -6.201429000 & 0.822342000 & -0.687557000 \\ \text { C } & -3.084705000 & 3.554384000 & -2.688720000 \\ \text { C } & -8.055288000 & -0.895205000 & -0.673283000 \\ \text { C } & -0.291566000 & 6.148106000 & -2.185550000 \\ \text { C } & -1.057829000 & 7.041219000 & 1.336329000 \\ \text { C } & -7.704323000 & 0.600838000 & -0.815940000 \\ \text { C } & -8.502623000 & 1.463400000 & 0.182270000\end{array}$




$\begin{array}{llll}\mathrm{H} & 2.658953000 & -6.878235000 & -4.703393000 \\ \mathrm{H} & 1.166905000 & -6.439895000 & -3.798650000 \\ \mathrm{H} & 2.75499000 & -6.445352000 & -2.969538000 \\ \mathrm{H} & 8.771701000 & -1.576824000 & 1.577126000 \\ \mathrm{H} & 7.335721000 & -1.141416000 & 2.564487000 \\ \mathrm{H} & 1.982677000 & -5.188605000 & 0.593487000 \\ \mathrm{H} & -1.048799000 & -6.175056000 & 4.103065000 \\ \mathrm{H} & -2.249555000 & -6.377073000 & 5.427496000 \\ \mathrm{H} & 1.832937000 & -4.329094000 & 2.162245000 \\ \mathrm{H} & 4.153210000 & -3.715833000 & 0.054586000 \\ \mathrm{H} & 7.122014000 & -2.064392000 & 1.045136000 \\ \mathrm{H} & -1.106213000 & -5.001093000 & 5.455180000 \\ \mathrm{H} & 2.887093000 & -4.545066000 & -1.602490000 \\ \mathrm{H} & 9.488935000 & 0.916184000 & 1.385344000 \\ \mathrm{H} & 3.920864000 & -2.913619000 & 1.636950000 \\ \mathrm{H} & 4.411303000 & -5.048911000 & -5.260714000 \\ \mathrm{H} & 8.118475000 & 1.375458000 & 2.436776000 \\ \mathrm{H} & 1.945366000 & -4.561441000 & -5.292450000 \\ \mathrm{H} & 0.454763000 & -4.342911000 & 1.019388000 \\ \mathrm{H} & 4.500329000 & -4.533802000 & -3.543407000 \\ \mathrm{H} & 2.172109000 & -2.459517000 & 3.346502000 \\ \mathrm{H} & 1.269584000 & -2.260235000 & 4.881537000 \\ \mathrm{H} & 3.996102000 & -1.928161000 & 0.145148000 \\ \mathrm{H} & 7.954003000 & -0.099994000 & -0.283567000 \\ \mathrm{H} & 8.300446000 & 2.145511000 & 0.828234000 \\ \mathrm{H} & 2.404163000 & -0.988207000 & 4.344389000 \\ \mathrm{H} & -3.363034000 & -5.475562000 & 3.385622000 \\ \mathrm{H} & 4.085571000 & -3.337622000 & -4.806747000 \\ \mathrm{H} & 4.058915000 & 0.389911000 & 4.312537000 \\ \mathrm{H} & -4.254020000 & -4.736524000 & 5.611273000 \\ \mathrm{H} & 1.674994000 & -2.140288000 & 0.990608000 \\ \mathrm{H} & -0.833966000 & -2.879267000 & 4.554485000 \\ \mathrm{H} & 5.871718000 & 1.075723000 & 2.668760000 \\ \mathrm{H} & -0.785727000 & -4.277398000 & -1.318238000\end{array}$




$\begin{array}{llll}\mathrm{H} & 3.881180000 & -1.786930000 & -2.343254000 \\ \mathrm{H} & -3.147462000 & -3.323805000 & 5.600580000 \\ \mathrm{H} & -2.350148000 & -5.061646000 & -0.905376000 \\ \mathrm{H} & 5.994528000 & -0.076012000 & -1.484703000 \\ \mathrm{H} & 4.947662000 & -0.700521000 & -3.303249000 \\ \mathrm{H} & 4.820369000 & 2.012053000 & 4.349831000 \\ \mathrm{H} & -3.193840000 & -4.478102000 & 1.318467000 \\ \mathrm{H} & 3.174096000 & 1.791043000 & 5.002644000 \\ \mathrm{H} & -2.173193000 & -4.157315000 & -2.444905000 \\ \mathrm{H} & 0.607913000 & -2.754189000 & -4.794019000 \\ \mathrm{H} & 3.261207000 & -1.005529000 & -3.822043000 \\ \mathrm{H} & 1.001898000 & -0.456271000 & 2.378725000 \\ \mathrm{H} & -4.452419000 & -3.437533000 & 4.380099000 \\ \mathrm{H} & -0.665082000 & -0.410658000 & 4.979001000 \\ \mathrm{H} & 2.210278000 & 1.374590000 & 2.811299000 \\ \mathrm{H} & 0.57884000 & 0.793040000 & 4.493586000 \\ \mathrm{H} & 2.310541000 & 0.178814000 & -1.961015000 \\ \mathrm{H} & -1.885107000 & -2.021515000 & -1.198917000 \\ \mathrm{H} & -4.446474000 & -3.482338000 & -0.300699000 \\ \mathrm{H} & -1.472634000 & -2.029221000 & -5.099450000 \\ \mathrm{H} & -2.392480000 & -2.242847000 & -3.576748000 \\ \mathrm{H} & 4.628149000 & 1.886843000 & -3.088647000 \\ \mathrm{H} & -0.922962000 & 0.618452000 & 3.531682000 \\ \mathrm{H} & 4.106256000 & 3.801694000 & 2.629789000 \\ \mathrm{H} & 0.540782000 & -0.259572000 & -5.122320000 \\ \mathrm{H} & 2.492812000 & 3.769187000 & 3.433345000 \\ \mathrm{H} & -4.170588000 & -2.661499000 & -1.866859000 \\ \mathrm{H} & -3.411282000 & -1.029094000 & 3.622209000 \\ \mathrm{H} & 3.056381000 & 1.586137000 & -3.910657000 \\ \mathrm{H} & -1.140766000 & -0.329386000 & -2.532356000 \\ \mathrm{H} & -4.085040000 & -1.679734000 & 2.105144000 \\ \mathrm{H} & -4.190420000 & -1.704395000 & -0.355249000 \\ \mathrm{H} & 2.618614000 & 3.556797000 & 1.656836000 \\ & 0.830428000 & 0.703919000 & -3.636684000\end{array}$




$\begin{array}{llll}\mathrm{H} & 3.105718000 & 2.515439000 & -2.372381000 \\ \mathrm{H} & -2.555899000 & -0.729783000 & -4.523765000 \\ \mathrm{H} & -2.418206000 & 0.231341000 & 1.838282000 \\ \mathrm{H} & -5.084385000 & -0.604919000 & 3.147007000 \\ \mathrm{H} & -0.656414000 & 0.976256000 & -4.601062000 \\ \mathrm{H} & -3.115750000 & 1.549346000 & 3.869961000 \\ \mathrm{H} & -2.272367000 & 1.574635000 & -2.882951000 \\ \mathrm{H} & -4.173961000 & 0.679867000 & -4.381599000 \\ \mathrm{H} & 2.243875000 & 5.992308000 & 1.954231000 \\ \mathrm{H} & 2.169912000 & 4.777121000 & -1.909028000 \\ \mathrm{H} & 2.879459000 & 5.881188000 & -0.686335000 \\ \mathrm{H} & -3.101694000 & 2.569638000 & 2.389643000 \\ \mathrm{H} & -4.661542000 & 1.975542000 & 3.054806000 \\ \mathrm{H} & -3.214698000 & 2.032096000 & -5.071987000 \\ \mathrm{H} & -0.136707000 & 5.261484000 & 2.126476000 \\ \mathrm{H} & -6.108383000 & 0.210184000 & 1.390104000 \\ \mathrm{H} & 1.26555600 & 7.159078000 & 2.908435000 \\ \mathrm{H} & 2.490408000 & 6.494462000 & -2.334367000 \\ \mathrm{H} & 1.741668000 & 7.561321000 & 1.232952000 \\ \mathrm{H} & -7.493319000 & -1.510310000 & -1.405874000 \\ \mathrm{H} & -4.847022000 & 2.340523000 & -4.420416000 \\ \mathrm{H} & -0.358057000 & 5.126813000 & -2.613330000 \\ \mathrm{H} & -7.799828000 & -1.268299000 & 0.341354000 \\ \mathrm{H} & -2.571955000 & 3.772735000 & -1.729220000 \\ \mathrm{H} & -2.469471000 & 3.985173000 & -3.506937000 \\ \mathrm{H} & 0.779325000 & 7.239497000 & -0.678445000 \\ \mathrm{H} & -5.963814000 & 1.512151000 & -2.718727000 \\ \mathrm{H} & -4.065444000 & 4.076586000 & -2.676549000 \\ \mathrm{H} & -1.241574000 & 7.491923000 & 2.333395000 \\ \mathrm{H} & -0.005894000 & 6.846930000 & -2.998837000 \\ \mathrm{H} & -1.979691000 & 6.515271000 & 1.015529000 \\ \mathrm{H} & -9.141044000 & -1.067048000 & -0.832545000 \\ \mathrm{H} & -1.298378000 & 6.429153000 & -1.815794000 \\ \mathrm{H} & -7.994870000 & 0.918706000 & -1.842736000\end{array}$




$\begin{array}{lrrr}\mathrm{H} & -0.868734000 & 7.874534000 & 0.626421000 \\ \mathrm{H} & -8.276228000 & 1.178124000 & 1.231632000 \\ \mathrm{H} & -8.259606000 & 2.539825000 & 0.067573000 \\ \mathrm{H} & -9.595456000 & 1.335269000 & 0.031071000 \\ \mathrm{~N} & 0.321710000 & 5.352880000 & 0.111239000 \\ \mathrm{~B} & 0.130231000 & 3.978057000 & 0.016882000 \\ \mathrm{Si} & -0.081036000 & -0.779735000 & -0.083556000 \\ \mathrm{Si} & 1.392353000 & 1.052237000 & 0.258757000 \\ \mathrm{Si} & -0.263260000 & 2.410358000 & 1.286932000 \\ \mathrm{Si} & -1.468825000 & 1.137268000 & -0.351219000 \\ \mathrm{Si} & 0.255155000 & 2.440918000 & -1.329754000\end{array}$


Supplementary Table 8: Atomic coordinates of the optimized structure of $\mathbf{1 0 .}$

$\begin{array}{lrrr}\text { C } & 8.384343000 & -0.119258000 & 0.027100000 \\ \text { C } & 3.478430000 & 2.318572000 & 3.142632000 \\ \text { C } & 7.562253000 & -0.966323000 & 1.020486000 \\ \text { C } & 3.684301000 & 0.138283000 & 4.420352000 \\ \text { C } & 5.348670000 & -0.122238000 & 1.968374000 \\ \text { C } & 7.860867000 & -2.471059000 & 0.860167000 \\ \text { C } & 6.071932000 & -0.671728000 & 0.896153000 \\ \text { C } & 3.273643000 & 0.790928000 & 3.087199000 \\ \text { C } & 3.974968000 & 0.178471000 & 1.874254000 \\ \text { C } & 5.375581000 & -0.940900000 & -0.296220000 \\ \text { C } & 3.293179000 & -0.064935000 & 0.644450000 \\ \text { C } & 4.006921000 & -0.652297000 & -0.446896000 \\ \text { C } & 3.809046000 & 0.090852000 & -2.855154000 \\ \text { C } & -0.601301000 & 0.320536000 & 4.149969000 \\ \text { C } & 3.359182000 & -0.941885000 & -1.802674000 \\ \text { C } & 0.549668000 & -1.940358000 & 4.259992000 \\ \text { C } & -0.226382000 & -0.953345000 & 3.369373000 \\ \text { C } & 3.611418000 & -2.374871000 & -2.308411000 \\ \text { C } & -3.200887000 & 2.918022000 & 2.506865000 \\ \text { C } & 2.376586000 & -3.752136000 & 1.095673000 \\ \text { C } & -1.840549000 & 3.975903000 & -3.108456000 \\ \text { C } & -1.456037000 & -1.578534000 & 2.713671000 \\ \text { C } & -3.455071000 & 1.595803000 & 1.754818000 \\ \text { C } & 0.885963000 & -3.487114000 & 0.808375000 \\ \text { C } & -2.968234000 & 1.980140000 & -0.759705000 \\ \text { C } & 0.163082000 & -0.220363000 & -4.217731000 \\ \text { C } & -2.472701000 & 2.571741000 & -3.218119000 \\ \text { C } & -3.898631000 & 1.894564000 & 0.322953000 \\ \text { C } & -1.684038000 & -1.547277000 & 1.305353000 \\ \text { C } & -2.398584000 & -2.193147000 & 3.559996000 \\ \text { C } & -0.032501000 & -2.191773000 & -1.247779000 \\ \text { C } & -3.440789000 & 2.345713000 & -2.057133000\end{array}$




$\begin{array}{lrrr}\text { C } & 0.579267000 & -3.356061000 & -0.685876000 \\ \text { C } & -4.410771000 & 0.684713000 & 2.542182000 \\ \text { C } & -0.031896000 & -4.537265000 & 1.465182000 \\ \text { C } & -5.263372000 & 2.138777000 & 0.080287000 \\ \text { C } & -0.885431000 & -0.953599000 & -3.360898000 \\ \text { C } & -0.266387000 & -2.157077000 & -2.652310000 \\ \text { C } & 0.895155000 & -4.449169000 & -1.513313000 \\ \text { C } & -4.819552000 & 2.565209000 & -2.248882000 \\ \text { C } & -3.081179000 & 2.364735000 & -4.614637000 \\ \text { C } & -5.749715000 & 2.454086000 & -1.200630000 \\ \text { C } & -7.858062000 & 3.737888000 & -0.575696000 \\ \text { C } & -3.565560000 & -2.802846000 & 3.068955000 \\ \text { C } & -2.885822000 & -2.130320000 & 0.797797000 \\ \text { C } & 2.556314000 & -5.865985000 & -3.773831000 \\ \text { C } & 0.091736000 & -3.268314000 & -3.444178000 \\ \text { C } & -3.966495000 & -4.439630000 & 4.982737000 \\ \text { C } & 0.661722000 & -4.429763000 & -2.899248000 \\ \text { C } & -5.362627000 & -2.319333000 & 4.765752000 \\ \text { C } & -7.242723000 & 2.629846000 & -1.453263000 \\ \text { C } & -3.784131000 & -2.759913000 & 1.683284000 \\ \text { C } & -4.592752000 & -3.423200000 & 4.008601000 \\ \text { C } & -2.126533000 & -1.317678000 & -4.196316000 \\ \text { C } & -3.248728000 & -2.127187000 & -0.688895000 \\ \text { C } & 1.033480000 & -5.617718000 & -3.778763000 \\ \text { C } & -4.621501000 & -1.483136000 & -0.967263000 \\ \text { C } & -3.148069000 & -3.548528000 & -1.279096000 \\ \text { C } & -7.985894000 & 1.289104000 & -1.270678000 \\ \text { C } & 0.255454000 & -6.886910000 & -3.375566000 \\ \text { H } & 9.473237000 & -0.289290000 & 0.164886000 \\ \text { H } & 8.181225000 & 0.963400000 & 0.159999000 \\ \text { H } & 4.555424000 & 2.563631000 & 3.266944000 \\ \text { H } & 7.869176000 & -0.670345000 & 2.049034000 \\ \text { H } & 4.715292000 & 0.421098000 & 4.721843000 \\ \text { H } & 8.134465000 & -0.380495000 & -1.023258000\end{array}$




$\begin{array}{lrrr}\mathrm{H} & 2.925008000 & 2.759357000 & 3.999292000 \\ \mathrm{H} & 5.882975000 & 0.080884000 & 2.910896000 \\ \mathrm{H} & 8.940292000 & -2.680260000 & 1.016865000 \\ \mathrm{H} & 3.125533000 & 2.815987000 & 2.215939000 \\ \mathrm{H} & 3.008021000 & 0.469905000 & 5.235987000 \\ \mathrm{H} & 3.643516000 & -0.968432000 & 4.361589000 \\ \mathrm{H} & 7.280666000 & -3.075027000 & 1.587752000 \\ \mathrm{H} & 7.595648000 & -2.827178000 & -0.158116000 \\ \mathrm{H} & 2.184406000 & 0.612286000 & 2.951610000 \\ \mathrm{H} & 5.919079000 & -1.384567000 & -1.146268000 \\ \mathrm{H} & 4.913876000 & 0.082341000 & -2.970325000 \\ \mathrm{H} & 3.506678000 & 1.119106000 & -2.568280000 \\ \mathrm{H} & 0.304515000 & 0.815188000 & 4.561238000 \\ \mathrm{H} & 1.465554000 & -1.462188000 & 4.659379000 \\ \mathrm{H} & 3.359962000 & -0.133950000 & -3.845243000 \\ \mathrm{H} & -1.118733000 & 1.051596000 & 3.496342000 \\ \mathrm{H} & -1.279393000 & 0.087808000 & 4.999088000 \\ \mathrm{H} & 4.663130000 & -2.523170000 & -2.634650000 \\ \mathrm{H} & -2.444606000 & 3.538978000 & 1.982967000 \\ \mathrm{H} & 0.456996000 & -0.639026000 & 2.548900000 \\ \mathrm{H} & -2.834989000 & 2.725773000 & 3.537943000 \\ \mathrm{H} & -0.054377000 & -2.274105000 & 5.129823000 \\ \mathrm{H} & 2.258784000 & -0.839380000 & -1.678809000 \\ \mathrm{H} & 3.011438000 & -2.931716000 & 0.704534000 \\ \mathrm{H} & -1.346183000 & 4.121416000 & -2.126606000 \\ \mathrm{H} & 0.853721000 & -2.843920000 & 3.695788000 \\ \mathrm{H} & 2.551831000 & -3.818471000 & 2.190230000 \\ \mathrm{H} & -4.138000000 & 3.510582000 & 2.579358000 \\ \mathrm{H} & -1.075628000 & 4.130928000 & -3.898673000 \\ \mathrm{H} & 3.380619000 & -3.129712000 & -1.533291000 \\ \mathrm{H} & 2.953136000 & -2.585723000 & -3.176065000 \\ \mathrm{H} & -2.619226000 & 4.761246000 & -3.213860000 \\ & 2.723470000 & -4.706184000 & 0.645531000 \\ 0.658962000 & -2.511824000 & 1.286077000 \\ & & & \end{array}$




\begin{tabular}{|c|c|c|c|}
\hline & 1.022346000 & 0.108591000 & -3.598539000 \\
\hline & -2.487633000 & 1.051359000 & 1.692402000 \\
\hline & -2.212144000 & -2.194273000 & 4.646169000 \\
\hline & -1.647609000 & 1.835312000 & -3.099308000 \\
\hline & -0.273327000 & 0.681719000 & -4.696892000 \\
\hline & -3.926188000 & 0.356260000 & 3.484030000 \\
\hline & 0.140596000 & -4.587792000 & 2.560572000 \\
\hline & 0.555837000 & -0.878748000 & -5.021785000 \\
\hline & -1.214307000 & -0.243480000 & -2.569530000 \\
\hline & -7.328202000 & 4.702606000 & -0.714762000 \\
\hline & -5.355340000 & 1.200826000 & 2.817619000 \\
\hline & -3.262006000 & -3.946149000 & 5.685645000 \\
\hline & -2.280071000 & 2.352696000 & -5.382707000 \\
\hline & -5.972712000 & 2.058470000 & 0.918363000 \\
\hline & 3.113559000 & -4.959665000 & -4.087559000 \\
\hline & -7.803089000 & 3.476441000 & 0.502485000 \\
\hline & 0.154722000 & -5.548368000 & 1.044702000 \\
\hline & 1.352138000 & -5.343259000 & -1.058636000 \\
\hline & -4.673922000 & -1.733001000 & 5.411092000 \\
\hline & -1.099737000 & -4.286293000 & 1.304232000 \\
\hline & 2.913303000 & -6.140556000 & -2.758277000 \\
\hline & -3.779548000 & 3.183858000 & -4.889160000 \\
\hline & -4.663060000 & -0.228313000 & 1.968306000 \\
\hline & -5.192671000 & 2.832038000 & -3.250160000 \\
\hline & -3.403404000 & -5.225458000 & 4.438581000 \\
\hline & -2.506785000 & -1.491843000 & -1.216951000 \\
\hline & -8.929346000 & 3.890674000 & -0.825391000 \\
\hline & -3.638993000 & 1.408735000 & -4.684896000 \\
\hline & -0.085730000 & -3.231860000 & -4.532304000 \\
\hline & 2.826701000 & -6.696172000 & -4.460352000 \\
\hline & -2.579156000 & -0.403861000 & -4.629670000 \\
\hline & -4.749959000 & -4.933478000 & 5.595430000 \\
\hline & -4.670515000 & -0.451238000 & -0.567119000 \\
\hline & -7.360492000 & 2.937879000 & -2.516718000 \\
\hline
\end{tabular}




$\begin{array}{llll}\mathrm{H} & -5.842405000 & -1.609339000 & 4.061388000 \\ \mathrm{H} & -6.151539000 & -2.753499000 & 5.416147000 \\ \mathrm{H} & -1.873784000 & -1.993392000 & -5.040376000 \\ \mathrm{H} & -2.896297000 & -1.822147000 & -3.580521000 \\ \mathrm{H} & 0.737391000 & -5.358077000 & -4.820245000 \\ \mathrm{H} & -2.135202000 & -3.974470000 & -1.131298000 \\ \mathrm{H} & -7.914775000 & 0.936361000 & -0.219570000 \\ \mathrm{H} & -4.702060000 & -3.219828000 & 1.280281000 \\ \mathrm{H} & -5.325846000 & -3.970385000 & 3.373997000 \\ \mathrm{H} & 0.524596000 & -7.212766000 & -2.348193000 \\ \mathrm{H} & -4.805684000 & -1.426994000 & -2.060778000 \\ \mathrm{H} & -5.453348000 & -2.068112000 & -0.520975000 \\ \mathrm{H} & -3.356153000 & -3.542861000 & -2.369275000 \\ \mathrm{H} & -9.063283000 & 1.391687000 & -1.520958000 \\ \mathrm{H} & -7.550915000 & 0.498997000 & -1.916797000 \\ \mathrm{H} & -3.884149000 & -4.227236000 & -0.797982000 \\ \mathrm{H} & 0.484026000 & -7.728557000 & -4.063279000 \\ \mathrm{H} & -0.839533000 & -6.709543000 & -3.394425000 \\ \mathrm{Si} & 1.238891000 & 3.989759000 & -0.204430000 \\ \mathrm{Si} & 0.288359000 & 2.335042000 & 1.243696000 \\ \mathrm{Si} & 1.488100000 & 0.482063000 & 0.389106000 \\ \mathrm{Si} & 0.930090000 & 1.979640000 & -1.435554000 \\ \mathrm{Si} & -1.165620000 & 1.444014000 & -0.452053000 \\ \mathrm{Si} & -0.479732000 & -0.795373000 & 0.001049000 \\ \mathrm{H} & 0.249903000 & 5.102928000 & -0.474324000 \\ \mathrm{~N} & 2.833176000 & 4.688749000 & 0.057612000 \\ \mathrm{C} & 2.963208000 & 5.916948000 & 0.881220000 \\ \mathrm{H} & 3.996132000 & 5.889997000 & 1.303843000 \\ \mathrm{C} & 2.000335000 & 5.924912000 & 2.079384000 \\ \mathrm{C} & 2.826617000 & 7.202019000 & 0.040369000 \\ \mathrm{H} & 3.565742000 & 7.221939000 & -0.786669000 \\ \mathrm{H} & 1.811888000 & 7.264497000 & -0.406897000 \\ \mathrm{H} & 2.987705000 & 8.109187000 & 0.660723000 \\ \mathrm{H} & 0.938889000 & 6.004290000 & 1.761102000\end{array}$




$\begin{array}{llll}\mathrm{H} & 2.211131000 & 6.800116000 & 2.727875000 \\ \mathrm{H} & 2.108604000 & 5.003901000 & 2.686689000 \\ \mathrm{C} & 4.044558000 & 4.342001000 & -0.735260000 \\ \mathrm{H} & 4.803244000 & 5.104903000 & -0.446280000 \\ \mathrm{C} & 3.832761000 & 4.478616000 & -2.254639000 \\ \mathrm{C} & 4.619342000 & 2.968468000 & -0.364062000 \\ \mathrm{H} & 4.869032000 & 2.908739000 & 0.712490000 \\ \mathrm{H} & 3.891129000 & 2.160842000 & -0.574548000 \\ \mathrm{H} & 5.536435000 & 2.744533000 & -0.947787000 \\ \mathrm{H} & 3.113225000 & 3.719055000 & -2.627539000 \\ \mathrm{H} & 4.787597000 & 4.329001000 & -2.801133000 \\ \mathrm{H} & 3.433626000 & 5.480698000 & -2.512913000\end{array}$


Supplementary Table 9: Atomic coordinates of the optimized structure of 9.

$\begin{array}{lrrr}\text { C } & 5.590711000 & -4.372327000 & -3.619296000 \\ \text { C } & 7.539100000 & 2.292282000 & 1.779653000 \\ \text { C } & 0.919956000 & -5.903560000 & 4.666994000 \\ \text { C } & 3.605412000 & -3.085988000 & 1.259081000 \\ \text { C } & 5.100434000 & -2.968588000 & -4.025408000 \\ \text { C } & 4.792791000 & -0.853266000 & 0.873818000 \\ \text { C } & 7.076446000 & 4.798796000 & 1.777442000 \\ \text { C } & 6.264742000 & -1.956732000 & -4.080319000 \\ \text { C } & 4.184992000 & -2.328199000 & -1.728604000 \\ \text { C } & 6.853384000 & 3.463629000 & 1.042316000 \\ \text { C } & 3.525256000 & -1.689342000 & 0.610065000 \\ \text { C } & 3.988179000 & -2.464091000 & -3.113785000 \\ \text { C } & 2.310562000 & -0.904880000 & 4.080368000 \\ \text { C } & -0.366188000 & -5.525113000 & 3.904450000 \\ \text { C } & 3.190823000 & -1.808678000 & -0.877849000 \\ \text { C } & 5.380056000 & 3.142167000 & 0.819214000 \\ \text { C } & -0.132792000 & -4.352358000 & 2.958134000 \\ \text { C } & -1.532471000 & -5.243849000 & 4.874503000 \\ \text { C } & 0.304633000 & -3.104797000 & 3.437500000 \\ \text { C } & 4.452725000 & 3.187294000 & 1.877382000 \\ \text { C } & 4.920929000 & 2.726332000 & -0.441311000 \\ \text { C } & 2.766942000 & 2.899993000 & 4.269392000 \\ \text { C } & 0.608989000 & -4.637299000 & -1.382720000 \\ \text { C } & 2.746460000 & -2.067412000 & -3.633420000 \\ \text { C } & -0.356592000 & -4.473759000 & 1.577753000 \\ \text { C } & 4.212239000 & 1.120483000 & -2.852835000 \\ \text { C } & 1.039044000 & -0.712950000 & 3.233099000 \\ \text { C } & 0.549431000 & -2.009563000 & 2.586033000 \\ \text { C } & 1.919210000 & -1.436996000 & -1.415338000 \\ \text { C } & 3.109048000 & 2.801907000 & 1.712957000 \\ \text { C } & -0.150538000 & -3.401056000 & 0.687770000 \\ \text { C } & 3.591301000 & 2.311147000 & -0.654487000\end{array}$




$\begin{array}{lrrr}\text { C } & 0.330013000 & -2.149810000 & 1.183129000 \\ \text { C } & 1.710848000 & -1.566228000 & -2.818441000 \\ \text { C } & 2.128372000 & 2.970407000 & 2.872835000 \\ \text { C } & 2.673200000 & 2.322296000 & 0.438679000 \\ \text { C } & -0.079195000 & -0.056507000 & 4.063531000 \\ \text { C } & -0.418586000 & -3.647882000 & -0.799292000 \\ \text { C } & 3.159321000 & 1.915188000 & -2.064931000 \\ \text { C } & -1.873448000 & -4.061906000 & -1.089570000 \\ \text { C } & 1.341220000 & 4.288968000 & 2.707566000 \\ \text { C } & 2.709968000 & 3.170448000 & -2.840771000 \\ \text { C } & 0.403335000 & -1.167348000 & -3.497767000 \\ \text { C } & -0.180787000 & -2.291234000 & -4.372153000 \\ \text { C } & 0.559741000 & 0.137114000 & -4.301320000 \\ \text { C } & -3.368604000 & -2.732054000 & 2.271866000 \\ \text { C } & -3.248507000 & -1.248130000 & 1.870107000 \\ \text { C } & -3.688299000 & -0.331726000 & 3.025596000 \\ \text { C } & -3.317967000 & -0.565129000 & -0.626887000 \\ \text { C } & -3.994715000 & -0.960615000 & 0.566538000 \\ \text { C } & -3.542288000 & 5.831054000 & 1.516966000 \\ \text { C } & -3.719026000 & -1.446678000 & -4.165182000 \\ \text { C } & -3.343274000 & -0.305872000 & -3.197711000 \\ \text { C } & -4.044253000 & -0.488852000 & -1.850706000 \\ \text { C } & -4.963549000 & 2.571764000 & 0.853347000 \\ \text { C } & -5.391443000 & -1.134194000 & 0.534042000 \\ \text { C } & -4.224545000 & 2.945678000 & -0.442006000 \\ \text { C } & -3.810002000 & 5.396235000 & 0.064664000 \\ \text { C } & -5.445106000 & -0.637160000 & -1.824171000 \\ \text { C } & -6.143762000 & -0.928499000 & -0.638370000 \\ \text { C } & -3.589577000 & 1.072044000 & -3.833734000 \\ \text { C } & -8.226588000 & -2.236398000 & 0.063025000 \\ \text { C } & -3.324275000 & 6.439083000 & -0.954904000 \\ \text { C } & -5.204381000 & 3.223263000 & -1.593415000 \\ \text { C } & -7.667627000 & -0.977362000 & -0.626228000 \\ \text { C } & -8.231174000 & 0.310759000 & 0.013546000\end{array}$




$\begin{array}{llll}\mathrm{H} & 6.357447000 & -4.745655000 & -4.330651000 \\ \mathrm{H} & 4.753043000 & -5.099464000 & -3.597746000 \\ \mathrm{H} & 6.052172000 & -4.361876000 & -2.608956000 \\ \mathrm{H} & 8.629259000 & 2.472181000 & 1.895327000 \\ \mathrm{H} & 7.104486000 & 2.160970000 & 2.793817000 \\ \mathrm{H} & 4.430651000 & -3.679542000 & 0.811138000 \\ \mathrm{H} & 1.754846000 & -6.112486000 & 3.966903000 \\ \mathrm{H} & 0.758671000 & -6.804870000 & 5.295492000 \\ \mathrm{H} & 3.794602000 & -3.011586000 & 2.350027000 \\ \mathrm{H} & 5.704961000 & -1.346517000 & 0.476033000 \\ \mathrm{H} & 7.397363000 & 1.338257000 & 1.231087000 \\ \mathrm{H} & 1.244521000 & -5.081407000 & 5.339768000 \\ \mathrm{H} & 5.154858000 & -2.619322000 & -1.293175000 \\ \mathrm{H} & 8.159410000 & 5.034616000 & 1.843596000 \\ \mathrm{H} & 4.941972000 & -0.712988000 & 1.964948000 \\ \mathrm{H} & 7.050307000 & -2.290219000 & -4.791245000 \\ \mathrm{H} & 6.685272000 & 4.762497000 & 2.816431000 \\ \mathrm{H} & 4.672548000 & -3.044610000 & -5.050584000 \\ \mathrm{H} & 2.659697000 & -3.647079000 & 1.118506000 \\ \mathrm{H} & 6.738862000 & -1.842298000 & -3.082022000 \\ \mathrm{H} & 3.135707000 & -1.341877000 & 3.485397000 \\ \mathrm{H} & 2.130758000 & -1.572814000 & 4.949066000 \\ \mathrm{H} & 4.714165000 & 0.151540000 & 0.413985000 \\ \mathrm{H} & 7.323281000 & 3.549349000 & 0.036537000 \\ \mathrm{H} & 6.570037000 & 5.636656000 & 1.255608000 \\ \mathrm{H} & 2.655650000 & 0.069299000 & 4.480320000 \\ \mathrm{H} & -0.654674000 & -6.397813000 & 3.276292000 \\ \mathrm{H} & 5.910066000 & -0.954452000 & -4.396492000 \\ \mathrm{H} & 3.428430000 & 2.016496000 & 4.378538000 \\ \mathrm{H} & -1.717004000 & -6.114095000 & 5.539593000 \\ \mathrm{H} & 2.693488000 & -1.138189000 & 1.098032000 \\ \mathrm{H} & 0.475226000 & -2.980432000 & 4.519391000 \\ \mathrm{H} & 4.792659000 & 3.532182000 & 2.865116000 \\ & 1.642466000 & -4.262654000 & -1.234750000\end{array}$




$\begin{array}{lrrr}\mathrm{H} & 4.596134000 & 0.265317000 & -2.263225000 \\ \mathrm{H} & -1.310169000 & -4.367904000 & 5.520686000 \\ \mathrm{H} & 0.527091000 & -5.633200000 & -0.897741000 \\ \mathrm{H} & 5.633565000 & 2.706396000 & -1.281609000 \\ \mathrm{H} & 5.073669000 & 1.751570000 & -3.159421000 \\ \mathrm{H} & 3.371741000 & 3.804329000 & 4.494534000 \\ \mathrm{H} & -0.709721000 & -5.441350000 & 1.182621000 \\ \mathrm{H} & 1.978425000 & 2.838187000 & 5.048057000 \\ \mathrm{H} & 0.453686000 & -4.777009000 & -2.472927000 \\ \mathrm{H} & 2.582236000 & -2.150893000 & -4.720853000 \\ \mathrm{H} & 3.759126000 & 0.707307000 & -3.777276000 \\ \mathrm{H} & 1.289954000 & -0.004465000 & 2.412407000 \\ \mathrm{H} & -2.469896000 & -5.025042000 & 4.323512000 \\ \mathrm{H} & -0.391380000 & -0.709692000 & 4.906406000 \\ \mathrm{H} & 1.391452000 & 2.140382000 & 2.796140000 \\ \mathrm{H} & 0.257300000 & 0.912660000 & 4.488826000 \\ \mathrm{H} & 2.274423000 & 1.248449000 & -1.957546000 \\ \mathrm{H} & -0.274887000 & -2.684770000 & -1.330746000 \\ \mathrm{H} & -2.144483000 & -5.017047000 & -0.592265000 \\ \mathrm{H} & 0.489587000 & -2.544018000 & -5.219919000 \\ \mathrm{H} & -0.344741000 & -3.217021000 & -3.785782000 \\ \mathrm{H} & 3.556440000 & 3.882340000 & -2.945620000 \\ \mathrm{H} & -0.974086000 & 0.139482000 & 3.439365000 \\ \mathrm{H} & 2.030136000 & 5.158774000 & 2.765716000 \\ \mathrm{H} & 1.260721000 & 0.000473000 & -5.152359000 \\ \mathrm{H} & 0.574865000 & 4.395196000 & 3.504546000 \\ \mathrm{H} & -2.023259000 & -4.201169000 & -2.181454000 \\ \mathrm{H} & -2.696605000 & -2.944202000 & 3.128481000 \\ \mathrm{H} & 2.356248000 & 2.903359000 & -3.859443000 \\ \mathrm{H} & -0.332244000 & -0.959150000 & -2.688119000 \\ \mathrm{H} & -3.066201000 & -3.401465000 & 1.444196000 \\ \mathrm{H} & -2.582986000 & -3.279321000 & -0.751015000 \\ \mathrm{H} & 0.817485000 & 4.337363000 & 1.730916000 \\ \mathrm{H} & 0.953646000 & 0.955401000 & -3.666186000\end{array}$




\begin{tabular}{|c|c|c|c|}
\hline & 1.885102000 & 3000 & -2.315829000 \\
\hline & -1.152445000 & -1.982594000 & -4.803452000 \\
\hline & -2.167466000 & -1.061478000 & 1.682656000 \\
\hline & -4.407286000 & -2.992543000 & 2.568595000 \\
\hline & -0.418337000 & 0.469281000 & -4.709969000 \\
\hline & -3.171516000 & -0.614653000 & 3.965712000 \\
\hline & -2.249647000 & -0.382786000 & -3.004272000 \\
\hline & -3.485254000 & -2.438135000 & -3.725947000 \\
\hline & -4.241146000 & 2.396817000 & 1.675732000 \\
\hline & -2.454108000 & 5.967796000 & 1.691046000 \\
\hline & -3.909064000 & 5.066181000 & 2.230996000 \\
\hline & -3.447016000 & 0.729425000 & 2.809590000 \\
\hline & -4.781383000 & -0.401389000 & 3.208561000 \\
\hline & -3.163840000 & -1.347857000 & -5.121307000 \\
\hline & -3.614359000 & 2.068551000 & -0.741303000 \\
\hline & -5.910672000 & -1.421994000 & 1.462412000 \\
\hline & -5.544701000 & 1.638641000 & 0.709117000 \\
\hline & -4.049686000 & 6.793225000 & 1.740185000 \\
\hline & -5.662827000 & 3.375751000 & 1.168032000 \\
\hline & -7.806494000 & -3.161417000 & -0.382529000 \\
\hline & -4.800706000 & -1.431733000 & -4.414221000 \\
\hline & -2.221657000 & 6.558310000 & -0.893505000 \\
\hline & -7.990200000 & -2.246574000 & 1.148170000 \\
\hline & -3.200226000 & 1.881431000 & -3.184281000 \\
\hline & -3.073436000 & 1.148195000 & -4.814153000 \\
\hline & -4.910535000 & 5.300757000 & -0.054312000 \\
\hline & -6.017167000 & -0.531975000 & -2.761939000 \\
\hline & -4.671687000 & 1.254720000 & -4.001768000 \\
\hline & -5.757164000 & 2.292131000 & -1.835902000 \\
\hline & -3.788406000 & 7.429198000 & -0.762448000 \\
\hline & -4.667051000 & 3.557193000 & -2.502751000 \\
\hline & -9.332073000 & -2.276769000 & -0.031049000 \\
\hline & -3.578050000 & 6.124040000 & -1.987286000 \\
\hline & -8.000310000 & -0.998390000 & -1.688550000 \\
\hline
\end{tabular}




$\begin{array}{llll}\mathrm{H} & -5.951560000 & 4.000153000 & -1.325974000 \\ \mathrm{H} & -7.917107000 & 0.389966000 & 1.076397000 \\ \mathrm{H} & -7.860233000 & 1.215381000 & -0.511137000 \\ \mathrm{H} & -9.341477000 & 0.321609000 & -0.016374000 \\ \mathrm{~N} & -3.258571000 & 4.046907000 & -0.237445000 \\ \mathrm{P} & -1.568002000 & 3.819743000 & -0.012275000 \\ \mathrm{Si} & 0.612353000 & -0.791860000 & -0.154762000 \\ \mathrm{Si} & 0.960026000 & 1.529544000 & 0.203172000 \\ \mathrm{Si} & -1.160714000 & 1.823839000 & 1.179816000 \\ \mathrm{Si} & -1.553428000 & 0.138485000 & -0.520858000 \\ \mathrm{Si} & -0.699151000 & 2.158323000 & -1.440333000\end{array}$


Supplementary Table 10: Atomic coordinates of the optimized structure of 8Tip.

$\begin{array}{lrrr}\text { C } & 7.427558000 & 1.769045000 & -3.582034000 \\ \text { C } & 1.025133000 & 8.151007000 & 2.219037000 \\ \text { C } & 5.903429000 & -3.430614000 & 4.653162000 \\ \text { C } & 5.349309000 & 0.755872000 & 1.319085000 \\ \text { C } & 6.017103000 & 2.314307000 & -3.885611000 \\ \text { C } & 4.102003000 & 2.988045000 & 1.110252000 \\ \text { C } & -1.373388000 & 8.078547000 & 1.358287000 \\ \text { C } & 5.973322000 & 3.852600000 & -3.777773000 \\ \text { C } & 4.988189000 & 1.822353000 & -1.601153000 \\ \text { C } & 0.081770000 & 7.625884000 & 1.117818000 \\ \text { C } & 4.126624000 & 1.493369000 & 0.739599000 \\ \text { C } & 4.963174000 & 1.662208000 & -2.997694000 \\ \text { C } & 2.835833000 & 0.735950000 & 4.233502000 \\ \text { C } & 4.887768000 & -4.163369000 & 3.754157000 \\ \text { C } & 4.012720000 & 1.248061000 & -0.766242000 \\ \text { C } & 0.168746000 & 6.110793000 & 0.970734000 \\ \text { C } & 4.115341000 & -3.199011000 & 2.860514000 \\ \text { C } & 3.925774000 & -5.036220000 & 4.587360000 \\ \text { C } & 3.384549000 & -2.126286000 & 3.402401000 \\ \text { C } & -0.155755000 & 5.256382000 & 2.041193000 \\ \text { C } & 0.578481000 & 5.522809000 & -0.237729000 \\ \text { C } & 0.290128000 & 3.513068000 & 4.422131000 \\ \text { C } & 4.775849000 & -2.514844000 & -1.461717000 \\ \text { C } & 3.922017000 & 0.895009000 & -3.544425000 \\ \text { C } & 4.082917000 & -3.367355000 & 1.467564000 \\ \text { C } & 2.398261000 & 4.139976000 & -2.267476000 \\ \text { C } & 1.922713000 & -0.085209000 & 3.304274000 \\ \text { C } & 2.662172000 & -1.221995000 & 2.599567000 \\ \text { C } & 2.973374000 & 0.447030000 & -1.332278000 \\ \text { C } & -0.103476000 & 3.854722000 & 1.924114000 \\ \text { C } & 3.351662000 & -2.506068000 & 0.625679000 \\ \text { C } & 0.626324000 & 4.125595000 & -0.414729000\end{array}$




$\begin{array}{lrrr}\text { C } & 2.642732000 & -1.400402000 & 1.184508000 \\ \text { C } & 2.933462000 & 0.281396000 & -2.746687000 \\ \text { C } & -0.426603000 & 3.007225000 & 3.155490000 \\ \text { C } & 0.250947000 & 3.274272000 & 0.667573000 \\ \text { C } & 0.688895000 & -0.611902000 & 4.059998000 \\ \text { C } & 3.375755000 & -2.787517000 & -0.878008000 \\ \text { C } & 1.047210000 & 3.584594000 & -1.781222000 \\ \text { C } & 2.848706000 & -4.190588000 & -1.233647000 \\ \text { C } & -1.943606000 & 2.885779000 & 3.393349000 \\ \text { C } & -0.059605000 & 3.831237000 & -2.822994000 \\ \text { C } & 1.852204000 & -0.530252000 & -3.459013000 \\ \text { C } & 2.431223000 & -1.630084000 & -4.367684000 \\ \text { C } & 0.890377000 & 0.385589000 & -4.237966000 \\ \text { C } & 0.845271000 & -4.668377000 & 2.128784000 \\ \text { C } & -0.238366000 & -3.688760000 & 1.642961000 \\ \text { C } & -1.355413000 & -3.532764000 & 2.690601000 \\ \text { C } & -0.860924000 & -3.159041000 & -0.819043000 \\ \text { C } & -0.824329000 & -4.068316000 & 0.282552000 \\ \text { C } & -0.855560000 & -3.461964000 & -4.563087000 \\ \text { C } & -1.374492000 & -2.715081000 & -3.319400000 \\ \text { C } & -1.386398000 & -3.597690000 & -2.070869000 \\ \text { C } & -1.371201000 & -5.355516000 & 0.128694000 \\ \text { C } & -1.938761000 & -4.890975000 & -2.166080000 \\ \text { C } & -1.957561000 & -5.780240000 & -1.078299000 \\ \text { C } & -2.751274000 & -2.080329000 & -3.591490000 \\ \text { C } & -1.576496000 & -8.288585000 & -0.935065000 \\ \text { C } & -2.593804000 & -7.160388000 & -1.200679000 \\ \text { C } & -3.825577000 & -7.284620000 & -0.279478000 \\ \text { H } & 8.178735000 & 2.200364000 & -4.277259000 \\ \text { H } & 7.459752000 & 0.664189000 & -3.677155000 \\ \text { H } & 7.742837000 & 2.026119000 & -2.548129000 \\ \text { H } & 0.995333000 & 9.259989000 & 2.270705000 \\ \text { H } & 0.733296000 & 7.762493000 & 3.217905000 \\ \text { H } & 6.292195000 & 1.128729000 & 0.865655000\end{array}$




$\begin{array}{lrrr}\mathrm{H} & 6.592489000 & -2.802011000 & 4.052386000 \\ \mathrm{H} & 6.511324000 & -4.154660000 & 5.235722000 \\ \mathrm{H} & 5.421044000 & 0.900904000 & 2.417296000 \\ \mathrm{H} & 4.962396000 & 3.540445000 & 0.676789000 \\ \mathrm{H} & 2.073504000 & 7.840295000 & 2.031552000 \\ \mathrm{H} & 5.391316000 & -2.766396000 & 5.381335000 \\ \mathrm{H} & 5.788009000 & 2.428712000 & -1.144609000 \\ \mathrm{H} & -1.444853000 & 9.186267000 & 1.397351000 \\ \mathrm{H} & 4.149052000 & 3.110843000 & 2.212875000 \\ \mathrm{H} & 6.710649000 & 4.320440000 & -4.464053000 \\ \mathrm{H} & -1.761869000 & 7.683006000 & 2.321038000 \\ \mathrm{H} & 5.771048000 & 2.043225000 & -4.937193000 \\ \mathrm{H} & 5.276839000 & -0.333992000 & 1.125775000 \\ \mathrm{H} & 6.214372000 & 4.188361000 & -2.746677000 \\ \mathrm{H} & 3.729276000 & 1.109918000 & 3.695717000 \\ \mathrm{H} & 3.190363000 & 0.135854000 & 5.097824000 \\ \mathrm{H} & 3.166597000 & 3.469163000 & 0.758913000 \\ \mathrm{H} & 0.415576000 & 8.066501000 & 0.151424000 \\ \mathrm{H} & -2.044295000 & 7.715036000 & 0.552885000 \\ \mathrm{H} & 2.287954000 & 1.608024000 & 4.640906000 \\ \mathrm{H} & 5.460097000 & -4.841930000 & 3.082221000 \\ \mathrm{H} & 4.967052000 & 4.246065000 & -4.028181000 \\ \mathrm{H} & 1.371760000 & 3.675845000 & 4.238074000 \\ \mathrm{H} & 4.486523000 & -5.757784000 & 5.218891000 \\ \mathrm{H} & 3.228444000 & 1.054117000 & 1.221041000 \\ \mathrm{H} & 3.383047000 & -1.981814000 & 4.495130000 \\ \mathrm{H} & -0.456754000 & 5.699086000 & 3.004514000 \\ \mathrm{H} & 5.080917000 & -1.464442000 & -1.278051000 \\ \mathrm{H} & 3.193124000 & 4.005233000 & -1.508894000 \\ \mathrm{H} & 3.304791000 & -4.408072000 & 5.261512000 \\ \mathrm{H} & 5.535890000 & -3.183357000 & -1.004640000 \\ \mathrm{H} & 0.860144000 & 6.181401000 & -1.076622000 \\ & 2.338531000 & 5.220006000 & -2.520943000 \\ -0.136584000 & 4.471450000 & 4.787086000\end{array}$




$\begin{array}{llll}\mathrm{H} & 4.641172000 & -4.208751000 & 1.022985000 \\ \mathrm{H} & 0.182115000 & 2.777320000 & 5.246694000 \\ \mathrm{H} & 4.791632000 & -2.684793000 & -2.558559000 \\ \mathrm{H} & 3.882027000 & 0.769141000 & -4.639547000 \\ \mathrm{H} & 2.718827000 & 3.593022000 & -3.177843000 \\ \mathrm{H} & 1.548144000 & 0.603574000 & 2.513991000 \\ \mathrm{H} & 3.235770000 & -5.608522000 & 3.934310000 \\ \mathrm{H} & 0.981316000 & -1.342979000 & 4.843644000 \\ \mathrm{H} & -0.050093000 & 1.982630000 & 2.946214000 \\ \mathrm{H} & 0.137653000 & 0.217153000 & 4.552698000 \\ \mathrm{H} & 1.176231000 & 2.483671000 & -1.680981000 \\ \mathrm{H} & 2.678211000 & -2.074710000 & -1.365854000 \\ \mathrm{H} & 3.475025000 & -4.994230000 & -0.792067000 \\ \mathrm{H} & 3.010063000 & -1.202301000 & -5.213308000 \\ \mathrm{H} & 3.106799000 & -2.306047000 & -3.807481000 \\ \mathrm{H} & -0.267620000 & 4.917068000 & -2.931778000 \\ \mathrm{H} & -0.013489000 & -1.116714000 & 3.366414000 \\ \mathrm{H} & -2.401644000 & 3.886543000 & 3.546451000 \\ \mathrm{H} & 1.422219000 & 0.938310000 & -5.041688000 \\ \mathrm{H} & -2.153018000 & 2.268207000 & 4.292525000 \\ \mathrm{H} & 2.848525000 & -4.334403000 & -2.334810000 \\ \mathrm{H} & 1.333795000 & -4.264061000 & 3.038869000 \\ \mathrm{H} & 0.236312000 & 3.439500000 & -3.818559000 \\ \mathrm{H} & 1.245067000 & -1.031055000 & -2.671936000 \\ \mathrm{H} & 1.635943000 & -4.810418000 & 1.367028000 \\ \mathrm{H} & 1.807355000 & -4.327002000 & -0.877537000 \\ \mathrm{H} & -2.448861000 & 2.406685000 & 2.530792000 \\ \mathrm{H} & 0.421761000 & 1.129954000 & -3.563701000 \\ \mathrm{H} & -1.002748000 & 3.332449000 & -2.522837000 \\ \mathrm{H} & 1.615784000 & -2.241288000 & -4.801831000 \\ \mathrm{H} & 0.257754000 & -2.698971000 & 1.528059000 \\ \mathrm{H} & 0.422765000 & -5.664267000 & 2.382518000 \\ & 0.075022000 & -0.204013000 & -4.708827000 \\ -0.936255000 & -3.260151000 & 3.681475000\end{array}$




$\begin{array}{llll}\mathrm{H} & -0.667902000 & -1.881412000 & -3.115061000 \\ \mathrm{H} & 0.107250000 & -3.972592000 & -4.356621000 \\ \mathrm{H} & -2.071932000 & -2.742029000 & 2.389689000 \\ \mathrm{H} & -1.924361000 & -4.479371000 & 2.809644000 \\ \mathrm{H} & -0.701641000 & -2.751996000 & -5.402844000 \\ \mathrm{H} & -1.350397000 & -6.047116000 & 0.986986000 \\ \mathrm{H} & -0.697506000 & -8.203356000 & -1.606465000 \\ \mathrm{H} & -1.574890000 & -4.230237000 & -4.917970000 \\ \mathrm{H} & -1.203867000 & -8.256989000 & 0.110954000 \\ \mathrm{H} & -3.072452000 & -1.440411000 & -2.745221000 \\ \mathrm{H} & -2.718778000 & -1.444303000 & -4.501497000 \\ \mathrm{H} & -2.371343000 & -5.223730000 & -3.124262000 \\ \mathrm{H} & -3.526420000 & -2.861847000 & -3.743302000 \\ \mathrm{H} & -2.040267000 & -9.285151000 & -1.093559000 \\ \mathrm{H} & -2.946703000 & -7.265378000 & -2.251352000 \\ \mathrm{H} & -3.533153000 & -7.201058000 & 0.789028000 \\ \mathrm{H} & -4.564729000 & -6.483843000 & -0.488266000 \\ \mathrm{H} & -4.327958000 & -8.265980000 & -0.414774000 \\ \mathrm{~B} & -3.026176000 & 0.518097000 & -0.085032000 \\ \mathrm{Si} & 1.709670000 & -0.285493000 & -0.078970000 \\ \mathrm{Si} & 0.093339000 & 1.402736000 & 0.363912000 \\ \mathrm{Si} & -1.522353000 & -0.099826000 & 1.185881000 \\ \mathrm{Si} & -0.366788000 & -1.343221000 & -0.539970000 \\ \mathrm{Si} & -1.404460000 & 0.605700000 & -1.360568000 \\ \mathrm{C} & -4.557768000 & 0.787744000 & -0.082425000 \\ \mathrm{C} & -5.501729000 & -0.235338000 & 0.306553000 \\ \mathrm{C} & -5.097220000 & 2.071567000 & -0.463675000 \\ \mathrm{C} & -6.881362000 & 0.037204000 & 0.290349000 \\ \mathrm{C} & -6.488598000 & 2.287380000 & -0.424290000 \\ \mathrm{C} & -7.407508000 & 1.292137000 & -0.059974000 \\ \mathrm{H} & -7.571496000 & -0.773819000 & 0.578280000 \\ \mathrm{H} & -6.871682000 & 3.283891000 & -0.704897000 \\ \mathrm{C} & -4.270986000 & 3.267805000 & -0.954134000 \\ \mathrm{H} & -4.964904000 & 4.136970000 & -0.920984000\end{array}$




$\begin{array}{llll}\mathrm{C} & -3.876246000 & 3.096718000 & -2.436940000 \\ \mathrm{C} & -3.089229000 & 3.635371000 & -0.046591000 \\ \mathrm{H} & -3.428302000 & 3.869968000 & 0.981566000 \\ \mathrm{H} & -2.354546000 & 2.807666000 & 0.030392000 \\ \mathrm{H} & -2.523564000 & 4.507410000 & -0.435472000 \\ \mathrm{H} & -3.203042000 & 2.226571000 & -2.581165000 \\ \mathrm{H} & -3.348540000 & 3.999712000 & -2.808934000 \\ \mathrm{H} & -4.776307000 & 2.934492000 & -3.064935000 \\ \mathrm{C} & -5.125114000 & -1.644313000 & 0.783536000 \\ \mathrm{H} & -6.068905000 & -2.232198000 & 0.738578000 \\ \mathrm{C} & -4.704292000 & -1.624647000 & 2.269113000 \\ \mathrm{C} & -4.125272000 & -2.373972000 & -0.122345000 \\ \mathrm{H} & -4.506135000 & -2.461430000 & -1.158975000 \\ \mathrm{H} & -3.153026000 & -1.841403000 & -0.172022000 \\ \mathrm{H} & -3.891918000 & -3.392335000 & 0.252420000 \\ \mathrm{H} & -3.783809000 & -1.023959000 & 2.420900000 \\ \mathrm{H} & -4.504496000 & -2.653089000 & 2.635796000 \\ \mathrm{H} & -5.504232000 & -1.179255000 & 2.895663000 \\ \mathrm{C} & -8.907396000 & 1.558488000 & -0.053320000 \\ \mathrm{H} & -9.050041000 & 2.625890000 & -0.335790000 \\ \mathrm{C} & -9.514327000 & 1.361231000 & 1.351295000 \\ \mathrm{C} & -9.634762000 & 0.695204000 & -1.105360000 \\ \mathrm{H} & -9.213144000 & 0.856054000 & -2.118767000 \\ \mathrm{H} & -9.539492000 & -0.386149000 & -0.869520000 \\ \mathrm{H} & -10.718066000 & 0.938099000 & -1.136877000 \\ \mathrm{H} & -9.425227000 & 0.304573000 & 1.682200000 \\ \mathrm{H} & -10.593643000 & 1.622721000 & 1.355840000 \\ \mathrm{H} & -9.000506000 & 1.993556000 & 2.104177000\end{array}$


Supplementary Table 11: Atomic coordinates of the optimized structure of 2.

\begin{tabular}{|c|c|c|c|}
\hline$n$ & -6.467679000 & 5.945015000 & 1.920427000 \\
\hline$C$ & -7.870427000 & 4.763139000 & 0.149893000 \\
\hline C & -6.999330000 & 4.589816000 & 1.412123000 \\
\hline C & -5.876808000 & 3.592003000 & 1.155282000 \\
\hline & -5.840788000 & 2.346875000 & 1.800013000 \\
\hline & -4.838888000 & 3.879716000 & 0.253645000 \\
\hline & -6.186243000 & -0.687764000 & 2.157996000 \\
\hline & -4.526903000 & 0.363030000 & 3.798475000 \\
\hline C & -8.413969000 & -3.811530000 & 0.060922000 \\
\hline C & -4.850182000 & 1.387442000 & 1.512694000 \\
\hline n & 0.212234000 & 8.304475000 & -0.223288000 \\
\hline C & -4.870634000 & 0.093648000 & 2.319993000 \\
\hline C & -3.813574000 & 2.963929000 & -0.053366000 \\
\hline C & -6.131706000 & 1.409483000 & -2.043089000 \\
\hline C & -2.152317000 & 4.824648000 & -0.520164000 \\
\hline C & -3.843377000 & 1.659987000 & 0.535700000 \\
\hline C & -1.558962000 & 3.119585000 & 2.907835000 \\
\hline C & 1.221013000 & 7.61 & 0.720 \\
\hline C & -3.196763000 & 3.585079000 & -2.457891000 \\
\hline C & -2.717185000 & 3.470859000 & -0.995266000 \\
\hline C & -7.600094000 & -3.716446000 & -1.246931000 \\
\hline C & 0.464163000 & 3.820361000 & 4.253108000 \\
\hline C & 0.657331000 & 5.348493000 & 1.744191000 \\
\hline C & 2.663613000 & 8.086187000 & 0.445352000 \\
\hline C & -6.171625000 & -1.646803000 & -1.692028000 \\
\hline$\checkmark$ & 1.092397000 & 6.100221000 & 0.640538000 \\
\hline$\checkmark$ & -4.937936000 & 0.462200000 & -2.257642000 \\
\hline C & -0.021095000 & 3.215549000 & 2.923273000 \\
\hline C & -6.345103000 & -2.877078000 & -1.040771000 \\
\hline C & -7.276677000 & -5.113919000 & -1.812354000 \\
\hline C & -5.052023000 & -0.826908000 & -1.450535000 \\
\hline & 0.495106000 & 3.949462000 & 1.684854000 \\
\hline
\end{tabular}




$\begin{array}{lrrr}\text { C } & -5.316326000 & -3.301664000 & -0.183718000 \\ \text { C } & -4.051177000 & -1.230023000 & -0.513587000 \\ \text { C } & 1.389056000 & 5.410160000 & -0.547648000 \\ \text { C } & -4.694415000 & 0.156747000 & -3.748845000 \\ \text { C } & -3.549849000 & -3.253913000 & 2.449441000 \\ \text { C } & -4.168162000 & -2.528333000 & 0.076744000 \\ \text { C } & 0.770918000 & 3.266403000 & 0.461325000 \\ \text { C } & 1.242411000 & 4.015298000 & -0.664070000 \\ \text { C } & -3.107834000 & -3.173722000 & 0.973119000 \\ \text { C } & 0.396873000 & 3.501128000 & -2.997908000 \\ \text { C } & 1.533253000 & -0.277050000 & 4.138213000 \\ \text { C } & 1.576410000 & 3.376841000 & -2.015429000 \\ \text { C } & -2.719497000 & -4.575125000 & 0.460288000 \\ \text { C } & 3.377722000 & 1.467700000 & 4.079537000 \\ \text { C } & 2.621516000 & 0.392517000 & 3.279182000 \\ \text { C } & 2.857575000 & 3.932457000 & -2.665425000 \\ \text { C } & 0.040610000 & -3.587165000 & 2.897657000 \\ \text { C } & 4.596329000 & 3.456668000 & 0.711945000 \\ \text { C } & -1.966548000 & -2.922453000 & -2.951187000 \\ \text { C } & 3.542782000 & -0.656638000 & 2.658470000 \\ \text { C } & 1.212679000 & -3.613834000 & 1.899504000 \\ \text { C } & 4.672529000 & 1.925903000 & 0.552469000 \\ \text { C } & 0.381375000 & -3.380821000 & -0.556475000 \\ \text { C } & 1.507826000 & 0.074203000 & -4.202570000 \\ \text { C } & -0.451773000 & -3.202303000 & -2.997243000 \\ \text { C } & 0.773110000 & -4.195592000 & 0.553738000 \\ \text { C } & 3.503934000 & -0.999493000 & 1.273912000 \\ \text { C } & 4.452913000 & -1.308339000 & 3.512677000 \\ \text { C } & 3.581739000 & 0.559151000 & -1.376309000 \\ \text { C } & -0.010191000 & -4.010095000 & -1.776888000 \\ \text { C } & 4.582034000 & 1.467117000 & -0.905828000 \\ \text { C } & 2.419365000 & -4.333898000 & 2.529513000 \\ \text { C } & 5.930563000 & 1.345575000 & 1.230495000 \\ \text { C } & 0.720752000 & -5.596530000 & 0.429014000\end{array}$




$\begin{array}{llll}\text { C } & 2.531100000 & -0.720935000 & -3.371600000 \\ \text { C } & 3.574185000 & 0.211528000 & -2.758499000 \\ \text { C } & 5.557030000 & 1.952818000 & -1.797009000 \\ \text { C } & -0.046048000 & -5.417259000 & -1.845592000 \\ \text { C } & -0.062516000 & -3.841596000 & -4.342150000 \\ \text { C } & 0.297964000 & -6.229605000 & -0.752887000 \\ \text { C } & -0.998570000 & -8.226744000 & 0.060740000 \\ \text { C } & 5.357673000 & -2.279169000 & 3.048622000 \\ \text { C } & 4.389364000 & -2.016325000 & 0.798058000 \\ \text { C } & 6.391212000 & 3.707565000 & -4.298182000 \\ \text { C } & 4.555293000 & 0.749056000 & -3.617207000 \\ \text { C } & 7.195381000 & -1.983454000 & 4.785632000 \\ \text { C } & 5.565240000 & 1.609913000 & -3.159627000 \\ \text { C } & 5.566805000 & -3.937768000 & 4.940440000 \\ \text { C } & 0.167508000 & -7.745874000 & -0.829770000 \\ \text { C } & 5.305146000 & -2.614233000 & 1.687036000 \\ \text { C } & 6.324327000 & -2.980143000 & 3.996271000 \\ \text { C } & 3.147757000 & -1.865374000 & -4.195241000 \\ \text { C } & 4.420670000 & -2.476213000 & -0.662160000 \\ \text { C } & 6.603220000 & 2.189877000 & -4.113330000 \\ \text { C } & 4.165549000 & -3.986427000 & -0.832123000 \\ \text { C } & 5.733525000 & -2.040609000 & -1.344350000 \\ \text { C } & 1.482443000 & -8.468761000 & -0.478715000 \\ \text { C } & 8.043081000 & 1.872438000 & -3.663270000 \\ \text { H } & -7.304866000 & 6.631391000 & 2.167902000 \\ \text { H } & -8.726599000 & 5.443678000 & 0.344270000 \\ \text { H } & -5.843623000 & 5.816915000 & 2.828715000 \\ \text { H } & -5.842534000 & 6.447534000 & 1.151852000 \\ \text { H } & -7.277197000 & 5.195552000 & -0.684043000 \\ \text { H } & -8.271080000 & 3.788428000 & -0.196962000 \\ \text { H } & -6.614790000 & 2.112473000 & 2.549910000 \\ \text { H } & -4.824066000 & 4.865684000 & -0.236858000 \\ \text { H } & -7.040624000 & -0.136220000 & 2.604869000 \\ \text { H } & -5.286268000 & 1.015579000 & 4.279017000 \\ & & & \\ & & 568\end{array}$




$\begin{array}{llll}\mathrm{H} & -8.658970000 & -2.803523000 & 0.454469000 \\ \mathrm{H} & -9.363638000 & -4.364804000 & -0.098889000 \\ \mathrm{H} & 0.257148000 & 9.409329000 & -0.118998000 \\ \mathrm{H} & -0.825440000 & 7.975318000 & -0.009006000 \\ \mathrm{H} & -2.884970000 & 5.648122000 & -0.655741000 \\ \mathrm{H} & -6.281371000 & 1.623426000 & -0.965855000 \\ \mathrm{H} & -2.020378000 & 4.129612000 & 2.938189000 \\ \mathrm{H} & -7.840235000 & -4.346183000 & 0.847778000 \\ \mathrm{H} & -6.411345000 & -0.867299000 & 1.087869000 \\ \mathrm{H} & -6.122535000 & -1.673446000 & 2.662796000 \\ \mathrm{H} & -4.486830000 & -0.586926000 & 4.372805000 \\ \mathrm{H} & -3.541298000 & 0.864220000 & 3.887670000 \\ \mathrm{H} & -4.132789000 & 4.179412000 & -2.520163000 \\ \mathrm{H} & -7.070986000 & 0.973934000 & -2.445151000 \\ \mathrm{H} & 0.963201000 & 7.912120000 & 1.762442000 \\ \mathrm{H} & -5.961366000 & 2.375388000 & -2.561105000 \\ \mathrm{H} & -1.862185000 & 4.793561000 & 0.546762000 \\ \mathrm{H} & -0.057811000 & 4.771900000 & 4.489915000 \\ \mathrm{H} & 0.428404000 & 8.057514000 & -1.284649000 \\ \mathrm{H} & -1.926984000 & 2.549756000 & 3.787077000 \\ \mathrm{H} & 0.428848000 & 5.880475000 & 2.681296000 \\ \mathrm{H} & -8.228319000 & -3.185782000 & -1.997466000 \\ \mathrm{H} & 2.754360000 & 9.185669000 & 0.573241000 \\ \mathrm{H} & -1.246633000 & 5.087867000 & -1.099769000 \\ \mathrm{H} & -1.935129000 & 2.611162000 & 1.999357000 \\ \mathrm{H} & 0.253972000 & 3.122150000 & 5.089994000 \\ \mathrm{H} & -4.054091000 & -0.551475000 & 1.931382000 \\ \mathrm{H} & -6.939315000 & -1.311352000 & -2.409387000 \\ \mathrm{H} & -3.390182000 & 2.595265000 & -2.913741000 \\ \mathrm{H} & -8.207634000 & -5.686854000 & -2.008757000 \\ \mathrm{H} & -2.434989000 & 4.095153000 & -3.081190000 \\ \mathrm{H} & -4.030579000 & 0.995010000 & -1.902515000 \\ & -1.878146000 & 2.742165000 & -0.960666000 \\ 1.553230000 & 4.028900000 & 4.239687000\end{array}$




$\begin{array}{lrrr}\mathrm{H} & -3.624974000 & -2.253441000 & 2.916479000 \\ \mathrm{H} & 3.383203000 & 7.595699000 & 1.132658000 \\ \mathrm{H} & -6.665956000 & -5.705898000 & -1.097962000 \\ \mathrm{H} & 2.974871000 & 7.844789000 & -0.593292000 \\ \mathrm{H} & -4.540077000 & -3.748716000 & 2.537907000 \\ \mathrm{H} & -5.550009000 & -0.392698000 & -4.195294000 \\ \mathrm{H} & -4.559210000 & 1.095846000 & -4.326570000 \\ \mathrm{H} & 0.368560000 & 2.174820000 & 2.864742000 \\ \mathrm{H} & -5.410396000 & -4.281845000 & 0.310177000 \\ \mathrm{H} & -6.706429000 & -5.041598000 & -2.761187000 \\ \mathrm{H} & 1.741607000 & 5.978250000 & -1.422755000 \\ \mathrm{H} & -2.825574000 & -3.846142000 & 3.043934000 \\ \mathrm{H} & 0.096233000 & 4.563526000 & -3.119315000 \\ \mathrm{H} & -0.483848000 & 2.929423000 & -2.646080000 \\ \mathrm{H} & 0.807059000 & 0.471838000 & 4.520251000 \\ \mathrm{H} & -3.784747000 & -0.464468000 & -3.879900000 \\ \mathrm{H} & 2.669799000 & 2.225808000 & 4.467120000 \\ \mathrm{H} & -2.192649000 & -2.544217000 & 0.919270000 \\ \mathrm{H} & -3.544349000 & -5.306328000 & 0.595267000 \\ \mathrm{H} & 0.675653000 & 3.110202000 & -3.998588000 \\ \mathrm{H} & 0.968769000 & -1.024397000 & 3.546350000 \\ \mathrm{H} & 1.977869000 & -0.801608000 & 5.011172000 \\ \mathrm{H} & 2.698819000 & 4.941738000 & -3.101980000 \\ \mathrm{H} & -0.767139000 & -2.916933000 & 2.545174000 \\ \mathrm{H} & 2.099310000 & 0.909490000 & 2.442674000 \\ \mathrm{H} & 0.375576000 & -3.221689000 & 3.890710000 \\ \mathrm{H} & 3.908754000 & 1.036601000 & 4.954026000 \\ \mathrm{H} & 1.754336000 & 2.294734000 & -1.837095000 \\ \mathrm{H} & 3.640949000 & 3.857234000 & 0.320270000 \\ \mathrm{H} & -2.264241000 & -2.403281000 & -2.019763000 \\ \mathrm{H} & 4.127910000 & 1.988925000 & 3.452953000 \\ \mathrm{H} & 4.657953000 & 3.733926000 & 1.785469000 \\ \mathrm{H} & -1.841801000 & -4.959680000 & 1.014544000 \\ & -2.448605000 & -4.555997000 & -0.611884000\end{array}$




$\begin{array}{lrrr}\mathrm{H} & -0.384595000 & -4.604293000 & 3.034631000 \\ \mathrm{H} & -2.276353000 & -2.282700000 & -3.804402000 \\ \mathrm{H} & 3.695592000 & 3.994415000 & -1.946692000 \\ \mathrm{H} & 3.177142000 & 3.258585000 & -3.486119000 \\ \mathrm{H} & -2.545458000 & -3.869144000 & -3.002728000 \\ \mathrm{H} & 5.430893000 & 3.969984000 & 0.188863000 \\ \mathrm{H} & 3.786178000 & 1.524815000 & 1.085914000 \\ \mathrm{H} & 1.014722000 & 0.849594000 & -3.583279000 \\ \mathrm{H} & 1.525965000 & -2.563100000 & 1.720673000 \\ \mathrm{H} & 4.459987000 & -1.039743000 & 4.581499000 \\ \mathrm{H} & 0.061183000 & -2.217383000 & -2.932401000 \\ \mathrm{H} & 0.715521000 & -0.592638000 & -4.604434000 \\ \mathrm{H} & 2.811552000 & -3.727520000 & 3.370835000 \\ \mathrm{H} & 5.950784000 & 1.595022000 & 2.311496000 \\ \mathrm{H} & 1.997386000 & 0.584405000 & -5.059660000 \\ \mathrm{H} & 1.970757000 & -1.183404000 & -2.527914000 \\ \mathrm{H} & -1.943650000 & -7.710720000 & -0.206672000 \\ \mathrm{H} & 2.142327000 & -5.330424000 & 2.935494000 \\ \mathrm{H} & 6.580059000 & -1.357805000 & 5.466833000 \\ \mathrm{H} & -0.201263000 & -3.112566000 & -5.167624000 \\ \mathrm{H} & 1.007300000 & -6.215255000 & 1.293718000 \\ \mathrm{H} & 5.367870000 & 3.928386000 & -4.664842000 \\ \mathrm{H} & -0.793098000 & -8.010579000 & 1.131063000 \\ \mathrm{H} & 6.852892000 & 1.759420000 & 0.770034000 \\ \mathrm{H} & 6.329589000 & 2.639256000 & -1.413468000 \\ \mathrm{H} & 4.865939000 & -3.375521000 & 5.594325000 \\ \mathrm{H} & 5.964796000 & 0.241792000 & 1.139939000 \\ \mathrm{H} & 6.521549000 & 4.246408000 & -3.335473000 \\ \mathrm{H} & -0.695513000 & -4.722454000 & -4.581670000 \\ \mathrm{H} & 3.245779000 & -4.470885000 & 1.807366000 \\ \mathrm{H} & -0.369143000 & -5.905201000 & -2.779060000 \\ \mathrm{H} & 7.741422000 & -1.299435000 & 4.103973000 \\ \mathrm{H} & 3.584205000 & -1.971305000 & -1.187860000 \\ & -1.155478000 & -9.321746000 & -0.039999000\end{array}$




\begin{tabular}{lrrr}
$\mathrm{H}$ & 0.994704000 & -4.176298000 & -4.348582000 \\
$\mathrm{H}$ & 4.538369000 & 0.481930000 & -4.687091000 \\
$\mathrm{H}$ & 7.119765000 & 4.127876000 & -5.023684000 \\
$\mathrm{H}$ & 2.355470000 & -2.552369000 & -4.551302000 \\
$\mathrm{H}$ & 7.940010000 & -2.518990000 & 5.411666000 \\
$\mathrm{H}$ & 3.172307000 & -4.275776000 & -0.436948000 \\
$\mathrm{H}$ & -0.087032000 & -7.999021000 & -1.883629000 \\
$\mathrm{H}$ & 4.969570000 & -4.676462000 & 4.367572000 \\
$\mathrm{H}$ & 6.270645000 & -4.491999000 & 5.597220000 \\
$\mathrm{H}$ & 3.682461000 & -1.487851000 & -5.091897000 \\
$\mathrm{H}$ & 3.868722000 & -2.455602000 & -3.595950000 \\
$\mathrm{H}$ & 6.445138000 & 1.704810000 & -5.103109000 \\
$\mathrm{H}$ & 5.890922000 & -0.947507000 & -1.253976000 \\
$\mathrm{H}$ & 1.775230000 & -8.286225000 & 0.577047000 \\
$\mathrm{H}$ & 5.997322000 & -3.384200000 & 1.306810000 \\
$\mathrm{H}$ & 7.005183000 & -3.597603000 & 3.367836000 \\
$\mathrm{H}$ & 8.281167000 & 2.363215000 & -2.695609000 \\
$\mathrm{H}$ & 4.187600000 & -4.258360000 & -1.908510000 \\
$\mathrm{H}$ & 4.937328000 & -4.599265000 & -0.320013000 \\
$\mathrm{H}$ & 5.721294000 & -2.291182000 & -2.425502000 \\
$\mathrm{H}$ & 1.376329000 & -9.566654000 & -0.607441000 \\
$\mathrm{H}$ & 2.316399000 & -8.122243000 & -1.123057000 \\
$\mathrm{H}$ & 6.605417000 & -2.555686000 & -0.887703000 \\
$\mathrm{H}$ & 8.780808000 & 2.236005000 & -4.409527000 \\
$\mathrm{H}$ & 8.190958000 & 0.780892000 & -3.530898000 \\
$\mathrm{Si}$ & -2.779501000 & 0.136270000 & -0.005929000 \\
$\mathrm{Si}$ & -0.801690000 & -0.285235000 & 1.323254000 \\
$\mathrm{Si}$ & 0.587345000 & 1.375152000 & 0.305840000 \\
$\mathrm{Si}$ & -0.793485000 & 0.317828000 & -1.370125000 \\
$\mathrm{H}$ & 0.433149000 & -1.482983000 & -0.384788000 \\
& 2.380771000 & -0.153819000 & -0.046159000 \\
\hline
\end{tabular}


Supplementary Table 12: Atomic coordinates of the optimized structure of 9тip.

\begin{tabular}{|c|c|c|c|}
\hline$C$ & -6.031921000 & 1.492084000 & -5.421443000 \\
\hline C & -7.419290000 & -3.933609000 & 2.251525000 \\
\hline C & -3.893954000 & 6.796818000 & 2.424496000 \\
\hline C & -4.962623000 & 2.407253000 & -0.191005000 \\
\hline ᄀ & -5.157608000 & 0.236879000 & -5.228923000 \\
\hline & -5.454512000 & -0.083612000 & 0.102826000 \\
\hline & -6.046758000 & -6.040400000 & 2.669222000 \\
\hline & -6.020697000 & -1.032063000 & -5.064539000 \\
\hline & -4.652070000 & 0.634142000 & -2.758253000 \\
\hline & -6.273548000 & -4.831167000 & 1.739407000 \\
\hline C & -4.401410000 & 0.973944000 & -0.281371000 \\
\hline C & -4.188565000 & 0.389943000 & -4.062707000 \\
\hline C & -3.807290000 & 1.711961000 & 3.405421000 \\
\hline C & -2.465503000 & 6.634326000 & 1.868847000 \\
\hline C & -3.780113000 & 0.725504000 & -1.657407000 \\
\hline C & -4.995931000 & -4.025051000 & 1.532527000 \\
\hline C & -2.194463000 & 5.216183000 & 1.379146000 \\
\hline C & -1.404856000 & 7.067947000 & 2.902 \\
\hline C & -2.387184000 & 4.103725000 & 2.217781000 \\
\hline C & -4.335217000 & -3.420097000 & 2.618979000 \\
\hline C & -4.448886000 & -3.852785000 & 0.250487000 \\
\hline C & -3.363350000 & -1.789180000 & 4.873398000 \\
\hline C & -1.950138000 & 3.892417000 & -2.832527000 \\
\hline C & -2.804101000 & 0.247490000 & -4.241788000 \\
\hline C & -1.700331000 & 4.977649000 & 0.087151000 \\
\hline C & -3.807803000 & -2.836834000 & -2.490173000 \\
\hline C & -2.397098000 & 1.656693000 & 2.790463000 \\
\hline C & -2.131905000 & 2.784920000 & 1.794053000 \\
\hline C & -2.368696000 & 0.609879000 & -1.858472000 \\
\hline C & -3.172497000 & -2.646299000 & 2.449939000 \\
\hline C & -1.402008000 & 3.680314000 & -0.373606000 \\
\hline C & -3.294669000 & -3.076063000 & 0.02442 \\
\hline
\end{tabular}




$\begin{array}{lrrr}\text { C } & -1.636066000 & 2.556740000 & 0.476063000 \\ \text { C } & -1.886830000 & 0.358256000 & -3.176145000 \\ \text { C } & -2.447127000 & -2.096445000 & 3.677098000 \\ \text { C } & -2.651987000 & -2.448713000 & 1.134010000 \\ \text { C } & -1.312421000 & 1.622767000 & 3.882540000 \\ \text { C } & -0.864878000 & 3.533161000 & -1.798581000 \\ \text { C } & -2.738091000 & -2.983387000 & -1.395976000 \\ \text { C } & 0.448468000 & 4.304327000 & -2.031553000 \\ \text { C } & -1.313393000 & -3.057787000 & 4.095680000 \\ \text { C } & -1.820283000 & -4.192427000 & -1.671618000 \\ \text { C } & -0.405093000 & 0.183180000 & -3.503532000 \\ \text { C } & 0.086416000 & 1.144153000 & -4.601445000 \\ \text { C } & -0.079962000 & -1.279253000 & -3.860608000 \\ \text { C } & 1.360985000 & 4.466576000 & 1.714305000 \\ \text { C } & 1.762442000 & 2.979199000 & 1.697837000 \\ \text { C } & 2.253026000 & 2.544541000 & 3.091156000 \\ \text { C } & 2.591770000 & 1.595449000 & -0.337249000 \\ \text { C } & 2.786892000 & 2.648700000 & 0.609823000 \\ \text { C } & 3.745148000 & 0.852778000 & -3.859622000 \\ \text { C } & 3.362678000 & 0.336237000 & -2.459704000 \\ \text { C } & 3.555914000 & 1.387531000 & -1.366622000 \\ \text { C } & 3.962853000 & 3.416957000 & 0.545117000 \\ \text { C } & 4.726204000 & 2.173932000 & -1.372955000 \\ \text { C } & 4.957866000 & 3.182815000 & -0.421770000 \\ \text { C } & 4.112124000 & -0.965854000 & -2.128497000 \\ \text { C } & 6.008968000 & 5.495904000 & -0.552852000 \\ \text { C } & 6.257077000 & 3.979904000 & -0.421238000 \\ \text { C } & 7.097720000 & 3.653454000 & 0.831551000 \\ \text { H } & -6.681296000 & 1.389116000 & -6.316439000 \\ \text { H } & -5.408103000 & 2.400827000 & -5.547438000 \\ \text { H } & -6.696114000 & 1.659591000 & -4.546881000 \\ \text { H } & -8.365864000 & -4.507920000 & 2.339657000 \\ \text { H } & -7.182962000 & -3.518741000 & 3.254666000 \\ \text { H } & -5.799426000 & 2.546867000 & -0.907975000\end{array}$




$\begin{array}{llll}\mathrm{H} & -4.653225000 & 6.481895000 & 1.679437000 \\ \mathrm{H} & -4.093326000 & 7.854034000 & 2.699414000 \\ \mathrm{H} & -5.348637000 & 2.622695000 & 0.826965000 \\ \mathrm{H} & -6.340006000 & -0.050097000 & -0.566910000 \\ \mathrm{H} & -7.593095000 & -3.077546000 & 1.567602000 \\ \mathrm{H} & -4.045277000 & 6.183475000 & 3.337971000 \\ \mathrm{H} & -5.735591000 & 0.738684000 & -2.585366000 \\ \mathrm{H} & -6.970466000 & -6.650711000 & 2.757473000 \\ \mathrm{H} & -5.815570000 & 0.092643000 & 1.137833000 \\ \mathrm{H} & -6.691600000 & -1.174688000 & -5.938153000 \\ \mathrm{H} & -5.764522000 & -5.714013000 & 3.692873000 \\ \mathrm{H} & -4.543914000 & 0.109695000 & -6.149205000 \\ \mathrm{H} & -4.181338000 & 3.158972000 & -0.421179000 \\ \mathrm{H} & -6.657481000 & -0.964315000 & -4.156704000 \\ \mathrm{H} & -4.591187000 & 1.721630000 & 2.622681000 \\ \mathrm{H} & -3.949699000 & 2.613493000 & 4.037577000 \\ \mathrm{H} & -5.029910000 & -1.106373000 & 0.065632000 \\ \mathrm{H} & -6.574668000 & -5.224816000 & 0.742516000 \\ \mathrm{H} & -5.234434000 & -6.693704000 & 2.289689000 \\ \mathrm{H} & -3.978241000 & 0.827877000 & 4.050933000 \\ \mathrm{H} & -2.368462000 & 7.306870000 & 0.986980000 \\ \mathrm{H} & -5.388380000 & -1.937109000 & -4.959107000 \\ \mathrm{H} & -4.252833000 & -1.199736000 & 4.570864000 \\ \mathrm{H} & -1.573762000 & 8.114502000 & 3.234582000 \\ \mathrm{H} & -3.593458000 & 0.880375000 & 0.474817000 \\ \mathrm{H} & -2.759613000 & 4.267188000 & 3.242004000 \\ \mathrm{H} & -4.740927000 & -3.559114000 & 3.632601000 \\ \mathrm{H} & -2.853634000 & 3.265155000 & -2.691393000 \\ \mathrm{H} & -4.514881000 & -2.017334000 & -2.256596000 \\ \mathrm{H} & -1.441005000 & 6.417266000 & 3.802808000 \\ \mathrm{H} & -2.249022000 & 4.958374000 & -2.743537000 \\ \mathrm{H} & -4.948894000 & -4.334246000 & -0.605387000 \\ \mathrm{H} & -4.389074000 & -3.771533000 & -2.639606000 \\ \mathrm{H} & -3.725338000 & -2.716383000 & 5.366548000\end{array}$




$\begin{array}{lrrr}\mathrm{H} & -1.531998000 & 5.838422000 & -0.581930000 \\ \mathrm{H} & -2.810154000 & -1.210727000 & 5.642224000 \\ \mathrm{H} & -1.583957000 & 3.728361000 & -3.867366000 \\ \mathrm{H} & -2.424397000 & 0.041860000 & -5.256940000 \\ \mathrm{H} & -3.323511000 & -2.587392000 & -3.456590000 \\ \mathrm{H} & -2.320026000 & 0.697415000 & 2.230821000 \\ \mathrm{H} & -0.381503000 & 6.997999000 & 2.480768000 \\ \mathrm{H} & -1.289369000 & 2.574905000 & 4.454660000 \\ \mathrm{H} & -1.964143000 & -1.142769000 & 3.366812000 \\ \mathrm{H} & -1.493746000 & 0.795767000 & 4.601090000 \\ \mathrm{H} & -2.111528000 & -2.065895000 & -1.444026000 \\ \mathrm{H} & -0.611122000 & 2.463431000 & -1.950905000 \\ \mathrm{H} & 0.319822000 & 5.398823000 & -1.894145000 \\ \mathrm{H} & -0.418261000 & 0.953415000 & -5.571654000 \\ \mathrm{H} & -0.098699000 & 2.201801000 & -4.328752000 \\ \mathrm{H} & -2.397332000 & -5.140007000 & -1.613081000 \\ \mathrm{H} & -0.309732000 & 1.467259000 & 3.437135000 \\ \mathrm{H} & -1.733052000 & -4.038183000 & 4.407127000 \\ \mathrm{H} & -0.599221000 & -1.588702000 & -4.792611000 \\ \mathrm{H} & -0.733939000 & -2.639169000 & 4.945563000 \\ \mathrm{H} & 0.811954000 & 4.140027000 & -3.067909000 \\ \mathrm{H} & 0.492770000 & 4.612414000 & 2.388797000 \\ \mathrm{H} & -1.367169000 & -4.126313000 & -2.683724000 \\ \mathrm{H} & 0.162824000 & 0.422913000 & -2.576177000 \\ \mathrm{H} & 1.062009000 & 4.821598000 & 0.710320000 \\ \mathrm{H} & 1.243217000 & 3.957218000 & -1.340559000 \\ \mathrm{H} & -0.603928000 & -3.245063000 & 3.263463000 \\ \mathrm{H} & -0.395595000 & -1.969348000 & -3.051777000 \\ \mathrm{H} & -0.997417000 & -4.250168000 & -0.928460000 \\ \mathrm{H} & 1.174585000 & 1.019564000 & -4.765462000 \\ \mathrm{H} & 0.835384000 & 2.407209000 & 1.466317000 \\ \mathrm{H} & 2.187688000 & 5.112579000 & 2.079491000 \\ & 1.011972000 & -1.417138000 & -4.013258000 \\ 1.516513000 & 2.824468000 & 3.872075000\end{array}$




$\begin{array}{lrrr}\mathrm{H} & 2.276121000 & 0.093972000 & -2.482172000 \\ \mathrm{H} & 3.270194000 & 1.830582000 & -4.080532000 \\ \mathrm{H} & 2.407233000 & 1.447555000 & 3.143476000 \\ \mathrm{H} & 3.214882000 & 3.040162000 & 3.341052000 \\ \mathrm{H} & 3.428339000 & 0.125248000 & -4.636066000 \\ \mathrm{H} & 4.113832000 & 4.217189000 & 1.287551000 \\ \mathrm{H} & 5.411258000 & 5.728859000 & -1.457964000 \\ \mathrm{H} & 4.84336000 & 0.977782000 & -3.967033000 \\ \mathrm{H} & 5.457253000 & 5.892683000 & 0.325744000 \\ \mathrm{H} & 3.782734000 & -1.388122000 & -1.160251000 \\ \mathrm{H} & 3.943073000 & -1.735113000 & -2.911165000 \\ \mathrm{H} & 5.489030000 & 1.998153000 & -2.148904000 \\ \mathrm{H} & 5.204156000 & -0.786845000 & -2.050169000 \\ \mathrm{H} & 6.969816000 & 6.048693000 & -0.618542000 \\ \mathrm{H} & 6.840425000 & 3.655663000 & -1.312414000 \\ \mathrm{H} & 6.560805000 & 3.949971000 & 1.757779000 \\ \mathrm{H} & 7.307076000 & 2.566710000 & 0.904071000 \\ \mathrm{H} & 8.066832000 & 4.195718000 & 0.813483000 \\ \mathrm{P} & 1.817405000 & -2.804722000 & 1.439182000 \\ \mathrm{Si} & -1.265238000 & 0.834832000 & -0.293969000 \\ \mathrm{Si} & -1.192278000 & -1.272051000 & 0.822657000 \\ \mathrm{Si} & 0.826635000 & -0.751886000 & 1.940920000 \\ \mathrm{Si} & 1.094918000 & 0.444348000 & -0.132370000 \\ \mathrm{Si} & 0.760018000 & -1.878454000 & -0.397458000 \\ \mathrm{C} & 3.628264000 & -2.769999000 & 0.938852000 \\ \mathrm{C} & 4.030760000 & -3.857180000 & 0.103017000 \\ \mathrm{C} & 4.603666000 & -1.802163000 & 1.339112000 \\ \mathrm{C} & 5.363970000 & -3.933208000 & -0.347675000 \\ \mathrm{C} & 5.923878000 & -1.944594000 & 0.874380000 \\ \mathrm{C} & 6.330572000 & -2.987216000 & 0.021213000 \\ \mathrm{H} & 5.643178000 & -4.761165000 & -1.016696000 \\ \mathrm{H} & 6.677603000 & -1.200390000 & 1.173423000 \\ \mathrm{C} & 3.052334000 & -4.928138000 & -0.387351000 \\ \mathrm{H} & 2.102337000 & -4.796584000 & 0.178351000\end{array}$




$\begin{array}{llll}\mathrm{C} & 3.544345000 & -6.358497000 & -0.096851000 \\ \mathrm{C} & 2.722008000 & -4.727491000 & -1.879364000 \\ \mathrm{H} & 2.323345000 & -3.707493000 & -2.065694000 \\ \mathrm{H} & 3.628709000 & -4.851191000 & -2.509711000 \\ \mathrm{H} & 1.961063000 & -5.460244000 & -2.222081000 \\ \mathrm{H} & 4.461190000 & -6.608851000 & -0.672042000 \\ \mathrm{H} & 2.767382000 & -7.100413000 & -0.377976000 \\ \mathrm{H} & 3.774832000 & -6.490295000 & 0.980193000 \\ \mathrm{C} & 4.266806000 & -0.631239000 & 2.258118000 \\ \mathrm{H} & 3.268875000 & -0.255590000 & 1.933403000 \\ \mathrm{C} & 5.230513000 & 0.557964000 & 2.140230000 \\ \mathrm{C} & 4.133692000 & -1.100348000 & 3.721033000 \\ \mathrm{H} & 3.395172000 & -1.922636000 & 3.821705000 \\ \mathrm{H} & 5.110141000 & -1.473268000 & 4.097183000 \\ \mathrm{H} & 3.804547000 & -0.265333000 & 4.375679000 \\ \mathrm{H} & 6.237964000 & 0.323920000 & 2.546062000 \\ \mathrm{H} & 4.842773000 & 1.421839000 & 2.713623000 \\ \mathrm{H} & 5.339219000 & 0.883594000 & 1.086425000 \\ \mathrm{C} & 7.760269000 & -3.007870000 & -0.513574000 \\ \mathrm{H} & 8.415187000 & -2.643512000 & 0.311297000 \\ \mathrm{C} & 7.897605000 & -2.010555000 & -1.686330000 \\ \mathrm{C} & 8.257838000 & -4.404283000 & -0.920715000 \\ \mathrm{H} & 8.120256000 & -5.141880000 & -0.103393000 \\ \mathrm{H} & 7.719233000 & -4.784151000 & -1.815091000 \\ \mathrm{H} & 9.336201000 & -4.373207000 & -1.181184000 \\ \mathrm{H} & 7.242880000 & -2.316319000 & -2.530082000 \\ \mathrm{H} & 8.943573000 & -1.966694000 & -2.057925000 \\ \mathrm{H} & 7.594681000 & -0.987400000 & -1.382014000\end{array}$


Supplementary Table 13: Comparison of Kohn-Sham frontier orbitals of 8, 9 and the hypothetical diisopropylamino substituted $\mathrm{Si}_{6}$ siliconoid 10 (level of theory: M06-2X/def2-SVPP//BP86/def2-SVP).

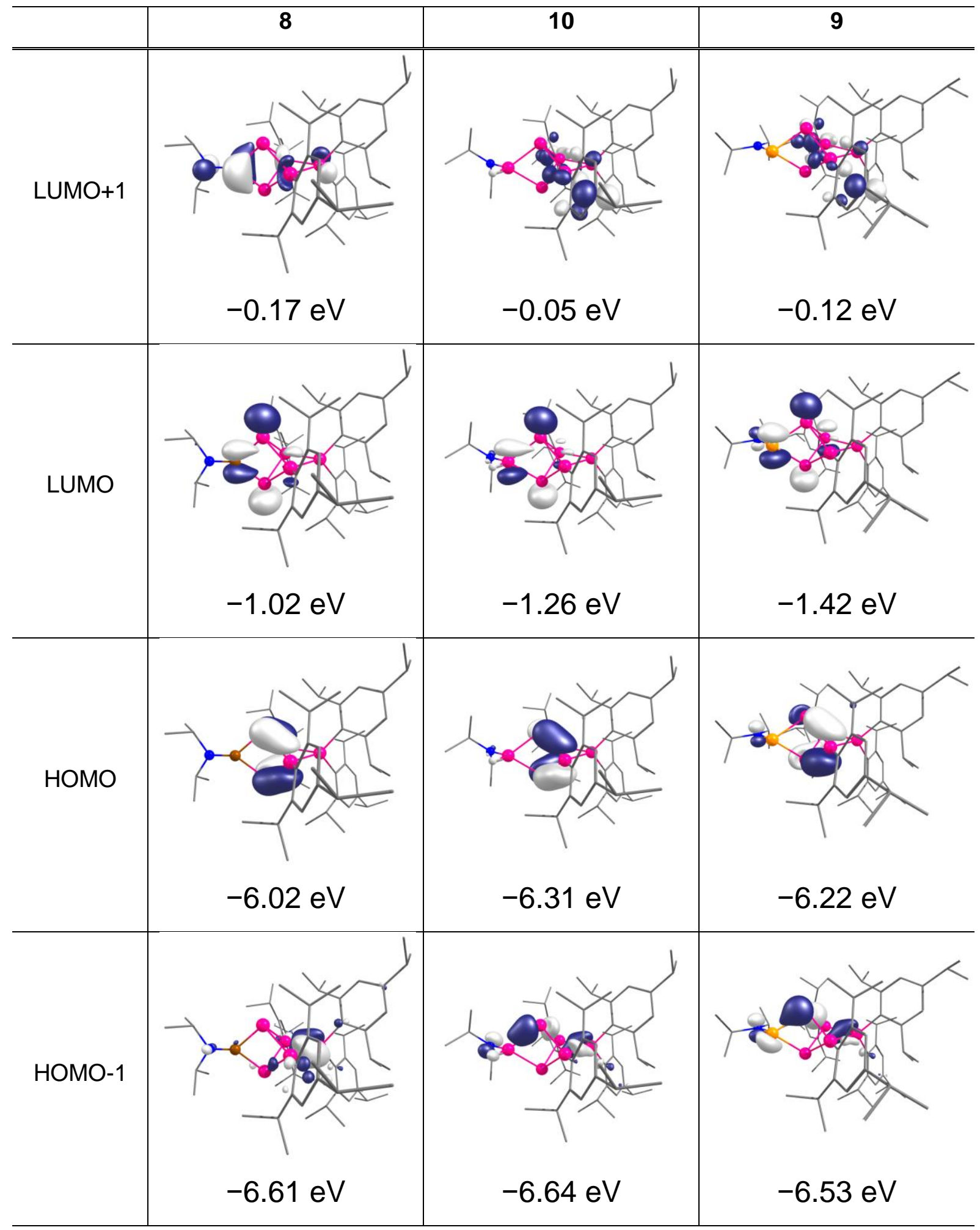




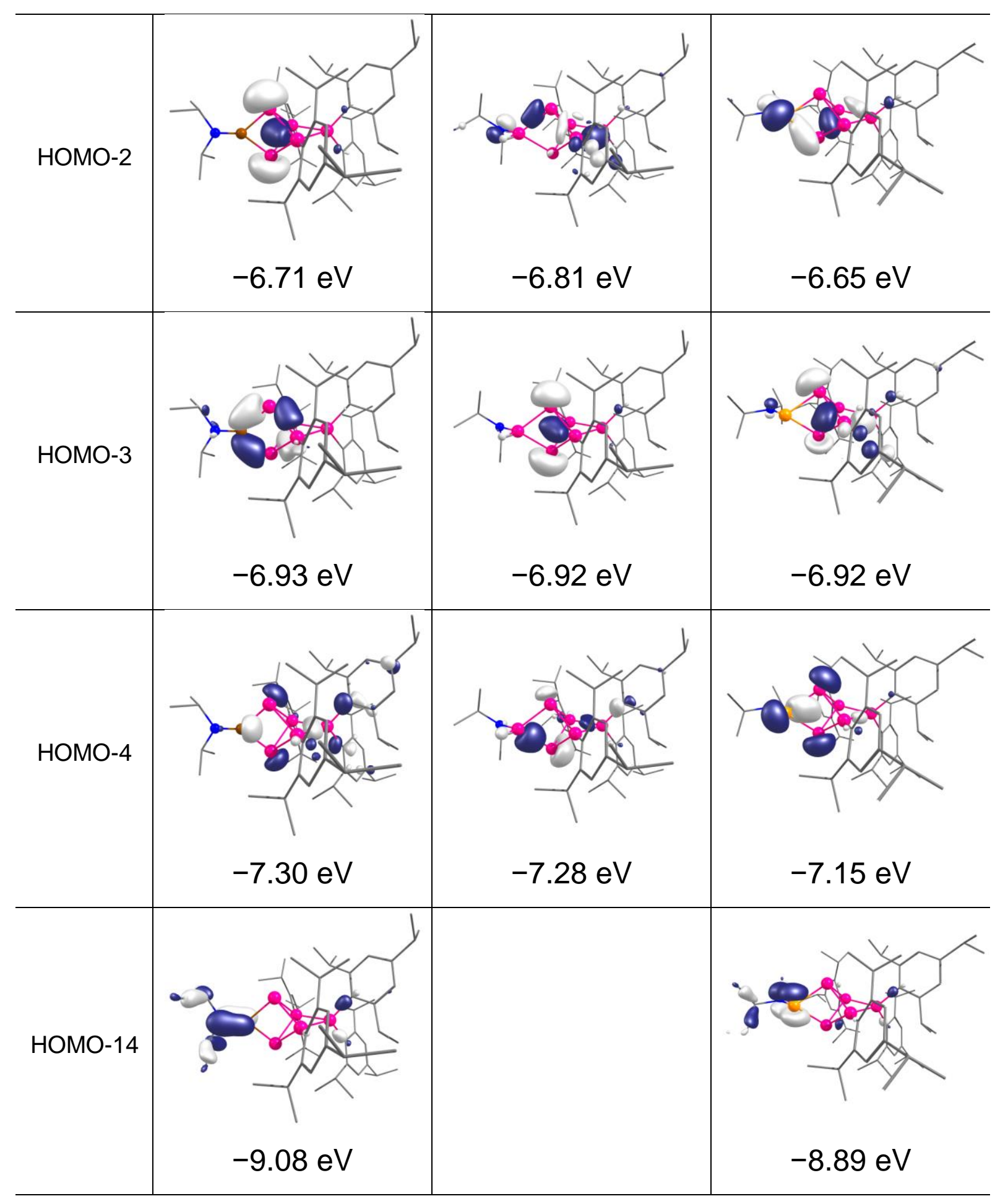


Supplementary Table 14: Comparison of Kohn-Sham frontier orbitals of the hypothetical all-Tip substituted doped siliconoids 8тір (BSisTip5), 9тір (PSisTip5) and hexasilabenzpolarene 2 (level of theory: M06-2X/def2-SVPP//BP86/def2-SVP).

LUMO




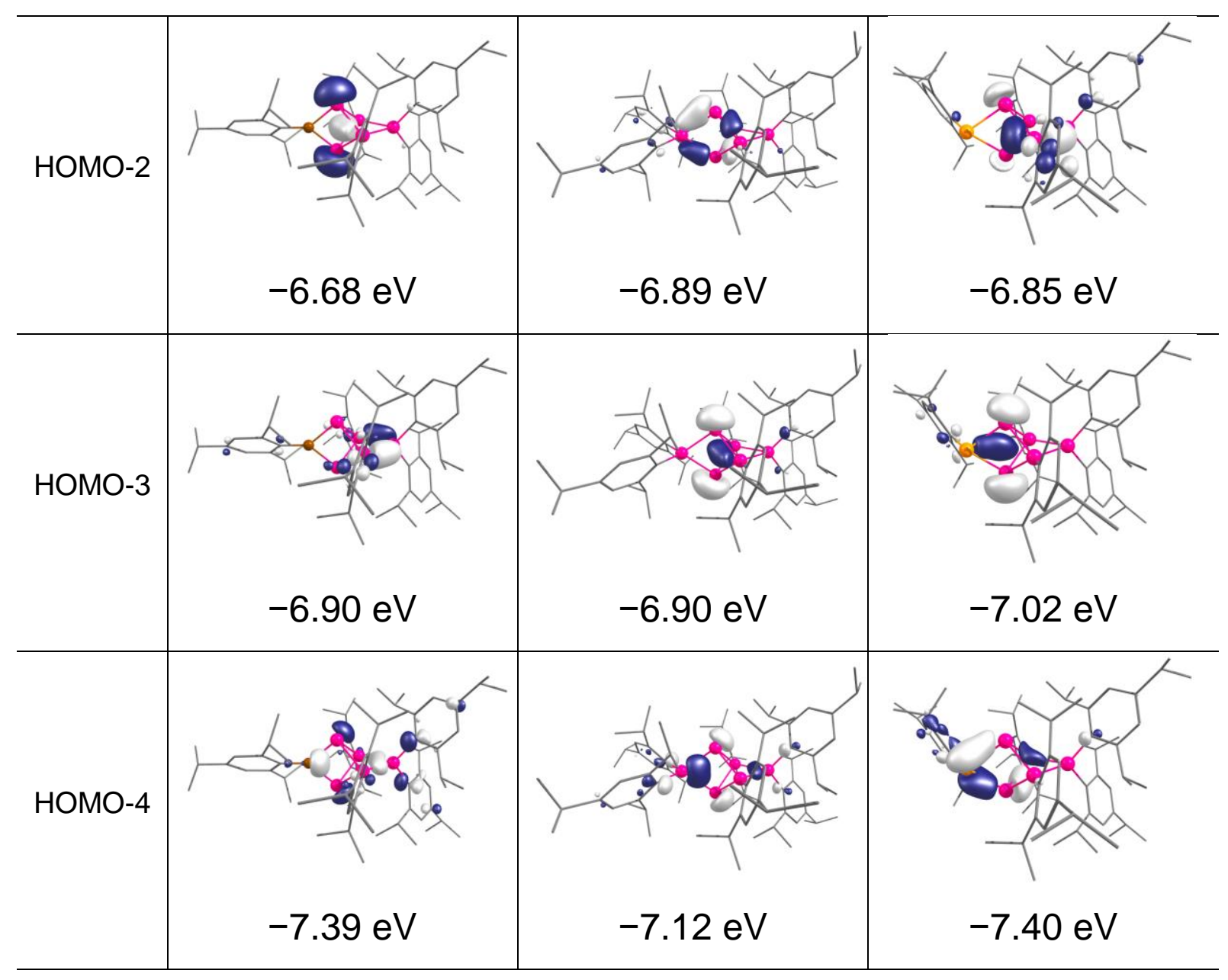


Supplementary Table 15: Comparison of selected calculated and experimentally observed bond lengths of $\mathbf{8}$ and $\mathbf{9}$ (level of theory: BP86/def2-SVP).

\begin{tabular}{c|cc|cc}
\hline & \multicolumn{2}{|c|}{$8(\mathrm{E}=\mathrm{B})$} & \multicolumn{2}{c}{$\mathbf{9}(\mathrm{E}=\mathrm{P})$} \\
& calc. $[\AA \AA]$ & exp. $[\AA \AA]$ & calc. $[\AA]$ & exp. $[\AA]$ \\
\hline \hline Si1-E & 2.0474 & $2.031(1)$ & 2.3568 & $2.314(2)$ \\
Si1-Si3 & 2.3726 & $2.3270(4)$ & 2.3780 & 2.391 (avg.) \\
Si1-Si5 & 2.3969 & $2.3602(4)$ & 2.4185 & $2.315(1)$ \\
Si1-Si2 & 2.6677 & $2.5911(4)$ & 2.6814 & $2.613(1)$ \\
Si2-E & 2.0556 & $2.038(1)$ & 2.3602 & $2.303(2)$ \\
Si2-Si3 & 2.3995 & $2.3617(4)$ & 2.4263 & $2.322($ avg.) \\
Si2-Si5 & 2.3754 & $2.3215(4)$ & 2.3533 & $2.370(1)$ \\
Si3-Si4 & 2.3817 & $2.3816(4)$ & 2.3854 & $2.382($ avg.) \\
Si4-Si5 & 2.3758 & $2.3594(4)$ & 2.3744 & $2.357(1)$ \\
E-N & 1.3913 & $1.374(2)$ & 1.7206 & $1.651(3)$ \\
\hline
\end{tabular}

Supplementary Table 16: Comparison of calculated and experimentally observed ${ }^{29} \mathrm{Si},{ }^{31} \mathrm{P}$ and ${ }^{11} \mathrm{~B}$ NMR chemical shifts of $\mathbf{8}$ and $\mathbf{9}$ ( $\delta$ values are given in ppm; level of theory: OLYP/def2-TZVPP//BP86/def2-SVP and OLYP/pcS-2//BP86/def2-SVP).

\begin{tabular}{c|ccc|ccc}
\hline & \multicolumn{3}{|c|}{$\mathbf{8}$} & \multicolumn{3}{c}{$\mathbf{9}$} \\
& $\delta_{\text {calc }}($ def2tzvpp) & $\delta_{\text {calc }}(\mathrm{pcS}-2)$ & $\delta_{\exp }$ & $\delta_{\text {calc }}($ def2tzvpp) & $\delta_{\text {calc }}(\mathrm{pcS}-2)$ & $\delta_{\text {exp }}$ \\
\hline \hline nudo-Si & $-290.9_{\text {avg }}$ & -281.6 avg & -288.6 & -227.9 & -221.9 & -240.0 \\
nudo-Si & & & & -219.6 & -214.8 & -225.8 \\
ligato-Si & $-39.9_{\text {avg }}$ & -37.4 avg & -43.3 & -66.4 & -64.6 & -76.7 \\
ligato-Si & 22.8 & 29.2 & 39.0 & -6.1 & -2.5 & 8.2 \\
remoto-Si & 75.6 & 77.1 & 83.3 & $/$ & 148.3 & 153.7 \\
privo-B & $/$ & $/$ & $/$ & 448.6 & 439.0 & 386.7 \\
privo-P & & & & & & \\
\hline
\end{tabular}


Supplementary Table 17: Comparison of calculated (4') and experimentally observed ${ }^{29} \mathrm{Si} \mathrm{NMR}$ chemical shifts of 4 ( $\delta$ values are given in ppm; level of theory: OLYP/def2-TZVPP//BP86/def2-SVP and OLYP/pcS-2//BP86/def2-SVP).

\begin{tabular}{|c|c|c|c|}
\hline & \multicolumn{2}{|c|}{$4^{\prime}$} & \multirow{2}{*}{$\begin{array}{c}4 \\
\delta_{\exp }\end{array}$} \\
\hline & $\delta_{\text {calc }}($ def2tzvpp $)$ & $\delta_{\text {calc }}(p c S-2)$ & \\
\hline $\begin{array}{l}\mathrm{Si}^{-} \\
\mathrm{Si}^{-}\end{array}$ & -248.2 avg & -237.4 avg & -298.3 \\
\hline $\begin{array}{l}\text { SiTip } \\
\text { SiTip }\end{array}$ & $-100.4_{\text {avg }}$ & $-98.3_{\mathrm{avg}}$ & -90.1 \\
\hline SiTip $_{2}$ & 25.5 & 28.6 & 55.7 \\
\hline
\end{tabular}




\section{References}

[1] Song, F., Zhang, J., Zhao, Y., Chen, W., Li, L. \& Xi, Z. Synthesis and antitumor activity of inositol phosphotriester analogues. Org. Biomol. Chem. 10, 3642-3654 (2012).

[2] Gaussian 09, Revision D.01, M. J. Frisch, G. W. Trucks, H. B. Schlegel, G. E. Scuseria, M. A. Robb, J. R. Cheeseman, G. Scalmani, V. Barone, B. Mennucci, G. A. Petersson, H. Nakatsuji, M. Caricato, X. Li, H. P. Hratchian, A. F. Izmaylov, J. Bloino, G. Zheng, J. L. Sonnenberg, M. Hada, M. Ehara, K. Toyota, R. Fukuda, J. Hasegawa, M. Ishida, T. Nakajima, Y. Honda, O. Kitao, H. Nakai, T. Vreven, J. A. Montgomery, Jr., J. E. Peralta, F. Ogliaro, M. Bearpark, J. J. Heyd, E. Brothers, K. N. Kudin, V. N. Staroverov, R. Kobayashi, J. Normand, K. Raghavachari, A. Rendell, J. C. Burant, S. S. lyengar, J. Tomasi, M. Cossi, N. Rega, J. M. Millam, M. Klene, J. E. Knox, J.B. Cross, V. Bakken, C. Adamo, J. Jaramillo, R. Gomperts, R. E. Stratmann, O. Yazyev, A. J. Austin, R. Cammi, C. Pomelli, J. W. Ochterski, R. L. Martin, K. Morokuma, V. G. Zakrzewski, G. A. Voth, P. Salvador, J. J. Dannenberg, S. Dapprich, A. D. Daniels, Ö. Farkas, J. B. Foresman, J. V. Ortiz, J. Cioslowski, and D. J. Fox, Gaussian, Inc., Wallingford CT, 2009.

[3] Becke, A. D. Density-functional exchange-energy approximation with correct asymptotic-behavior. Phys. Rev. A 38, 3098-3100 (1988).

[4] Perdew, J. P. Density-functional approximation for the correlation energy of the inhomogeneous electron gas. Phys. Rev. B 33, 8822-8824 (1986).

[5] Grimme, S., Antony, J., Ehrlich S. and Krieg H. A consistent and accurate ab initio parameterization of density functional dispersion correction (DFT-D) for the 94 elements H-Pu. J. Chem. Phys. 132, 154104 (2010).

[6] Weigend, F. and Ahlrichs, R. Balanced basis sets of split valence, triple zeta valence and quadruple zeta valence quality for $\mathrm{H}$ to $\mathrm{Rn}$ : Design and assessment of accuracy. Phys. Chem. Chem. Phys. 7, 3297-3305 (2005).

[7] http://www.chemcraftprog.com.

[8] Zhao, Y. and Truhlar, D. G. The M06 suite of density functionals for main group thermochemistry, thermochemical kinetics, noncovalent interactions, excited states, and transition elements: two new functionals and systematic 
testing of four M06-class functionals and 12 other functionals. Theor. Chem. Acc. 120, 215-241 (2008).

[9] London, F. The quantic theory of inter-atomic currents in aromatic combinations. J. Phys. Radium. 8, 397-409 (1937).

[10] McWeeny, R. Perturbation theory for Fock-Dirac density matrix. Phys. Rev. 126, 1028 (1962).

[11] Ditchfield, R. Self-consistent perturbation theory of diamagnetism. 1. Gaugeinvariant LCAO method for N.M.R. chemical shifts. Mol. Phys. 27, 789-807 (1974).

[12] Wolinski, K., Hilton, J. F., and Pulay, P. Efficient implementation of the Gauge-independent atomic orbitalm for NMR chemical shift calculations. J. Am. Chem. Soc. 112, 8251-8260 (1990).

[13] Cheeseman, J. R., Trucks, G. W., Keith, T. A., and Frisch, M. J. A comparison of models for calculating nuclear magnetic resonance shielding tensors. J. Chem. Phys. 104, 5497-5509 (1996).

[14] Handy, N. C. and Cohen, A. J. Left-right correlation energy. Mol. Phys. 99, 403-412 (2001).

[15] Hoe, W.-M., Cohen, A., and Handy, N. C. Assessment of a new local exchange functional OPTX. Chem. Phys. Lett. 341, 319-328 (2001).

[16] Lee, C., Yang, W. and Parr, R. G. Development of the Colle-Salvetti correlation-energy formula into a functional of the electron density. Phys. Rev. B 37, 785-789 (1988).

[17] Miehlich, B., Savin, A., Stoll, H. and Preuss, H. Results obtained with the correlation-energy density functionals of Becke and Lee, Yang and Parr. Chem. Phys. Lett. 157, 200-206 (1989).

[18] Weigend, F. Accurate Coulomb-fitting basis sets for $\mathrm{H}$ to Rn. Phys. Chem. Chem. Phys. 8, 1057-1065 (2006).

[19] Feller, D. The role of databases in support of computational chemistry calculations. J. Comp. Chem. 17, 1571-1586 (1996).

[20] Schuchardt, K. L., Didier, B. T., Elsethagen, T., Sun, L., Gurumoorthi, V., Chase, J. and Windus, T. L. Basis set exchange: a community database for computational sciences. J. Chem. Inf. Model. 47, 1045-1052 (2007). 
[21] Jensen, F. Basis set convergence of nuclear magnetic shielding constants calculated by density functional methods. J. Chem. Theory Comput., 4, 719727 (2008). 